\title{
2009
}

\section{Argonne National Laboratory Annual Illness and Injury Surveillance Report}

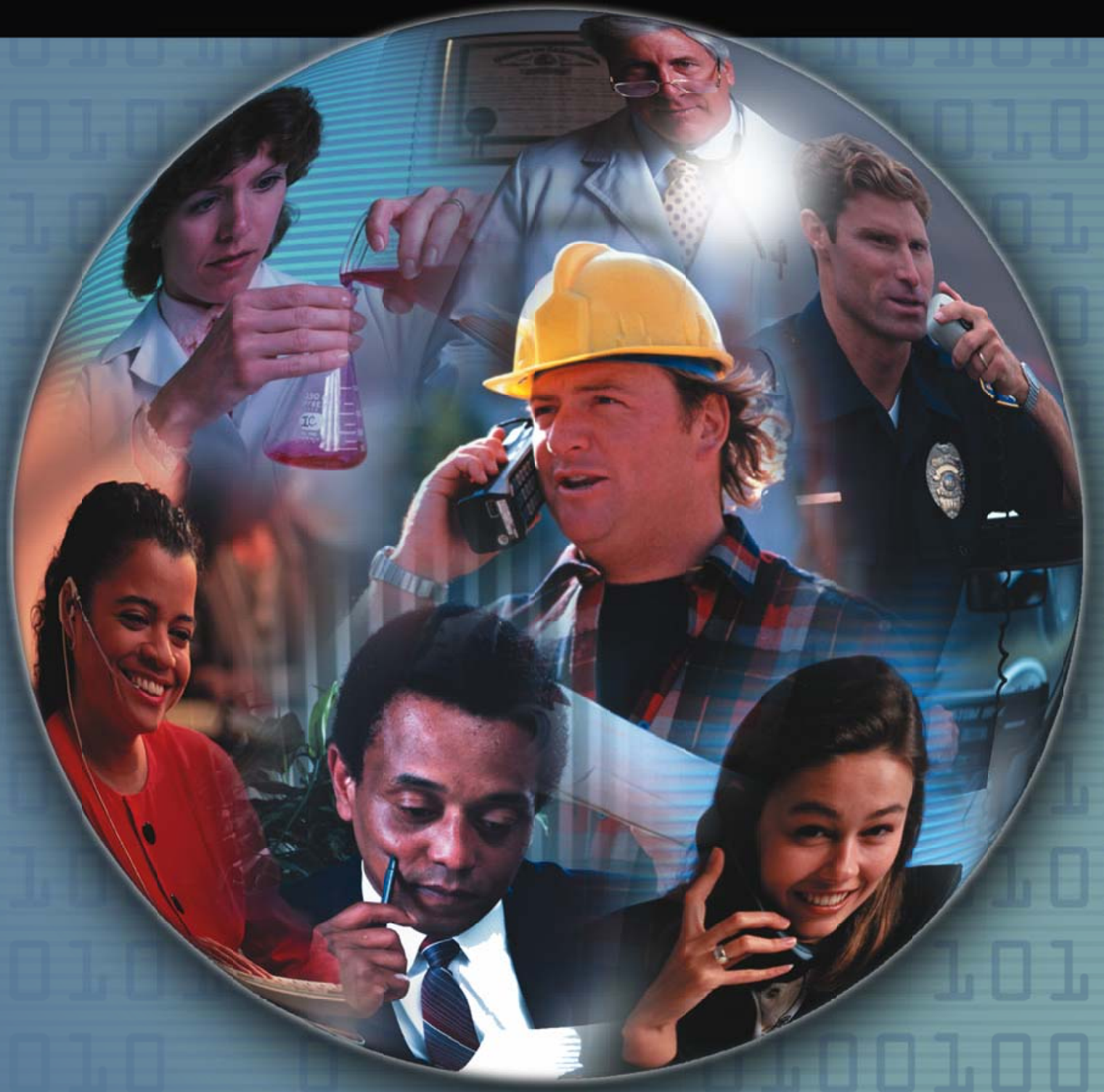




\section{Argonne National Laboratory 2009 Illness and Injury Surveillance Report}

Questions or comments about this report or the Illness and Injury Surveillance Program (IISP) may be directed to:

E-mail:

Dr. Cliff Strader at cliff.strader@hq.doe.gov or Dr. Bonnie Richter at bonnie.richter@hq.doe.gov

or direct letters to:

Mail Stop HS-13 / GTN Building

U.S. Department of Energy

1000 Independence Avenue, S.W.

Washington, DC 20585-0270

Additional information about the Department of Energy's Office of Illness and Injury Prevention Programs, the IISP, and annual reports for DOE sites participating in this program can be found at:

http://www.hss.energy.gov/healthsafety/WSHP/epi/surv/

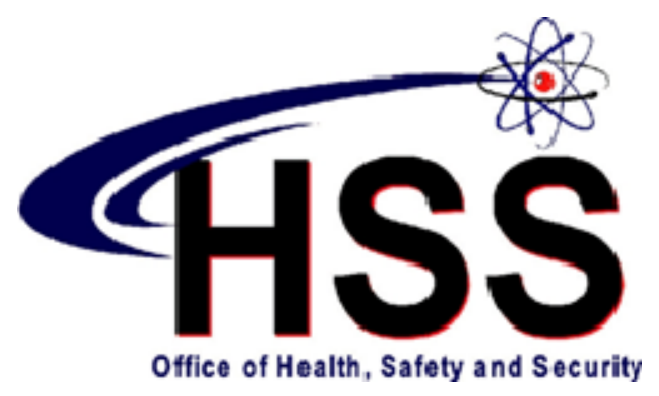

ACKNOWLEDGEMENT

LifeART images copyright 2000 Lippincott Williams \& Wilkins. All rights reserved.

This document was produced under contract number DE-AC05-06OR23100 between the

U.S. Department of Energy and Oak Ridge Associated Universities. 


\title{
Argonne National Laboratory
}

\section{Illness and Injury Surveillance Report}

\begin{abstract}
At A Glance
The year 2009 marks the first year of participation in the Illness and Injury Surveillance Program (IISP) for Argonne National Laboratory (ANL).
\end{abstract}

A total of 2,804 ANL employees were included in illness and injury surveillance in 2009. There were 859 (31 percent) women and 1,945 (69 percent) men in the work force. The average age of women in the work force was 48 years and 49 years for men. Sixty-eight percent of the workers were in the Professional job category.

Service workers had the highest absence rate among both male and female workers. Men in the Professional category and women in the Security and Fire category had the lowest absence rates. The 3 women Security and Fire workers reported no absences in 2009, and among the remaining occupational groups, the lowest absence rate was for female Professional workers.

Among both women and men, the most common diagnoses included conditions affecting the musculoskeletal and respiratory systems, injuries, and unspecified symptoms. Musculoskeletal conditions, respiratory diagnoses, and injuries are consistently among the more common diagnoses at many IISP sites.

Women lost 3,183 calendar days due to illness and injury. Respiratory conditions (28 percent), unspecified symptoms (13 percent), and musculoskeletal conditions (13 percent) accounted for 54 percent of all reported diagnoses among women.

Men lost 5,160 calendar days due to illness and injury. Fifty percent of all reported diagnoses among men were due to respiratory conditions ( 22 percent), musculoskeletal conditions (15 percent), and injuries (13 percent).

Seven sentinel health events for occupations were reported in 2009. Four possible occupational events were due to carpal tunnel syndrome, reported by female workers aged 50 and above. Two of the women were Service workers with the remaining 2 women in the Professional and Administrative Support occupational groups. These 4 events were responsible for 352 days absent. 
There were 9 OSHA-recordable events among women and 22 OSHA-recordable events among men. The rate of OSHA-recordable events was the same for men and women (1 per 100 workers). Sprains and strains were the most common type of injury among both men and women.

Security and Fire workers and Line Operators had the highest rate of OSHA events among women (33 per 100 workers). Among men, Security and Fire workers had the highest rate (14 per 100 workers).

Injuries were responsible for the largest percentage of OSHA diagnoses for both women (57 percent) and men (76 percent). The most frequent cause of accidents was overexertion and strenuous movements, followed by falls.

The initial year of health data provide a limited opportunity for interpretation, but overall rates and patterns appear consistent with those observed at other sites in the IISP. 
The Argonne National Laboratory

Work Force - 2009

The Work Force by Gender and Age 1

The Work Force by Gender and Job

Category .. 1

Number and Length of Absences

Absence Rate by Gender and Age 2

Number of Days Absent by

Gender and Age 2

Absence Rate by Job Category

and Gender 3

Average Duration of Absence by

Job Category and Gender.

Diagnostic Categories

Number of Diagnoses and Lost Calendar Days by Diagnostic Category

(Categorized by ICD-9-CM) and Gender 4

Common Diagnoses Among Female

Workers in 2009 . 5

Common Diagnoses Among Male

Workers in 2009 . .. 6

Number of Most Frequently Reported Diagnoses by Job Category and Gender .. 7

\section{Rates of Disease Occurrence}

Rates for All Illnesses and Injuries Combined by Job Category, Gender, and Age .. 8

Rates for Selected Diagnostic Categories by Job Category, Gender, and Age

\section{Sentinel Health Events for Occupations (SHEOs)}

Characteristics of SHEOs by Gender 11

SHEO Diagnoses by Gender 11

Occupational Safety and Health Administration (OSHA)-Recordable Events

OSHA-Recordable Events by Gender and Age.

OSHA-Recordable Events by Job

Category and Gender

\section{Diagnostic and Accident Categories for OSHA-Recordable Events}

OSHA-Recordable Diagnoses by

Diagnostic Category and Gender 13

OSHA-Recordable Accidents by Type and Gender

Rates of OSHA-Recordable Events

OSHA-Recordable Rates by Age and Job Categories Among Women, All Diagnoses Combined 14

OSHA-Recordable Rates by Age and Job Categories Among Men, All Diagnoses Combined .14

\section{Appendices}

Appendices A-W 16 
The Argonne National Laboratory Work Force - 2009

Figure 1. The Work Force by Gender and Age

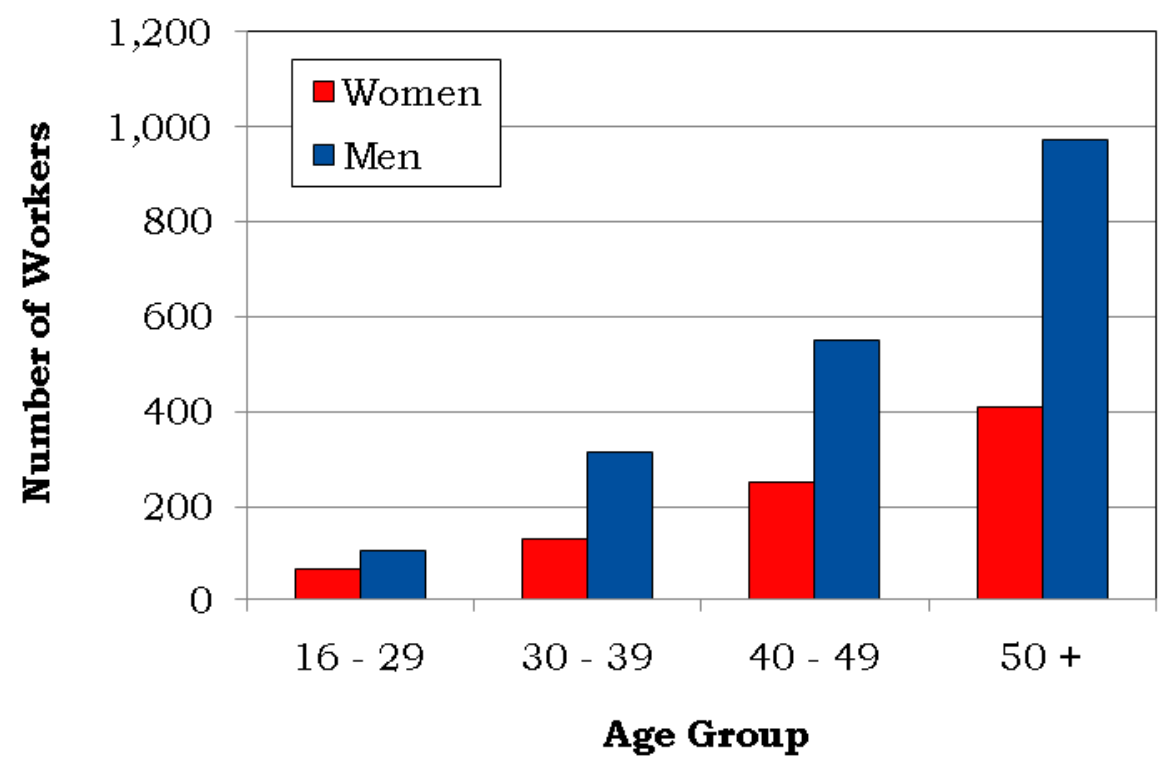

Figure 2. The Work Force by Gender and Job Category

\begin{tabular}{|l|c|c|}
\hline \multicolumn{1}{|c|}{ Job Category } & W omen & M en \\
\hline \multirow{2}{*}{ Professional } & 463 & 1,455 \\
& $53 \%$ & $\mathbf{7 5 \%}$ \\
\hline \multirow{2}{*}{ Administrative Support } & 310 & 48 \\
& $36 \%$ & $2 \%$ \\
\hline \multirow{2}{*}{ Technical Support } & 31 & 168 \\
& $4 \%$ & $9 \%$ \\
\hline \multirow{2}{*}{ Service } & 31 & 63 \\
& $4 \%$ & $3 \%$ \\
\hline \multirow{2}{*}{ Crafts } & 3 & 14 \\
& $<1 \%$ & $1 \%$ \\
\hline \multirow{2}{*}{ Line Operators } & 12 & 183 \\
& $1 \%$ & $9 \%$ \\
\hline \multirow{2}{*}{ Total } & 9 & 14 \\
& $1 \%$ & $1 \%$ \\
\hline
\end{tabular}




\section{Number and Length of Absences}

Figure 3. Absence Rate by Gender and Age

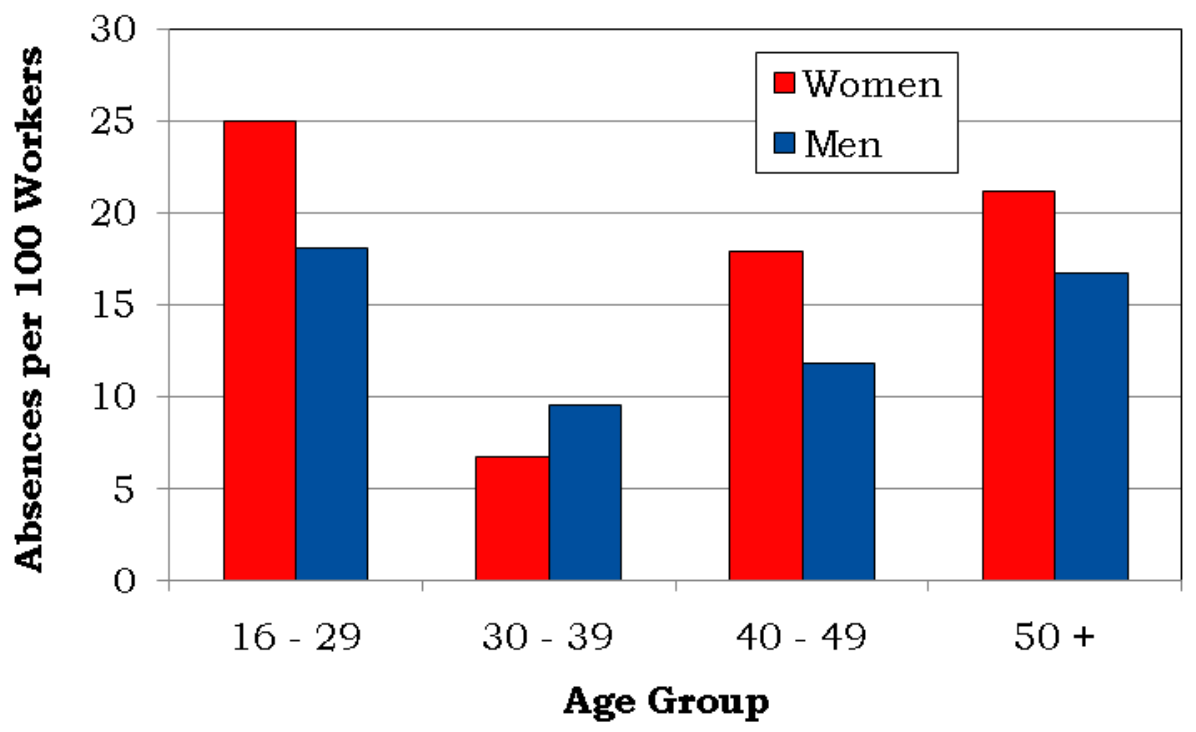

Figure 4. Number of Days Absent by Gender and Age

\begin{tabular}{|c|c|c|c|c|}
\hline \multirow{3}{*}{ Gender } & \multirow{2}{*}{ Age } & \multirow{2}{*}{$\begin{array}{c}\text { Number of } \\
\text { Absences }\end{array}$} & \multicolumn{2}{|c|}{ Number of Days Absent } \\
\cline { 3 - 5 } & & 16 & Total & Average \\
\hline \multirow{4}{*}{ Women* } & $16-29$ & 9 & 152 & 10 \\
\cline { 2 - 5 } & $30-39$ & 45 & 107 & 12 \\
\cline { 2 - 5 } & $40-49$ & 730 & 16 \\
\cline { 2 - 5 } & $50+$ & 87 & 2,194 & 25 \\
\cline { 2 - 5 } & Total & 157 & 3,183 & 20 \\
\hline \multirow{3}{*}{ Men } & $16-29$ & 19 & 284 & 15 \\
\cline { 2 - 5 } & $30-39$ & 30 & 544 & 18 \\
\cline { 2 - 5 } & $40-49$ & 65 & 1,082 & 17 \\
\cline { 2 - 5 } & $50+$ & 163 & 3,250 & 19 \\
\cline { 2 - 5 } & Total & 277 & 5,160 & 20 \\
\hline
\end{tabular}

*Normal pregnancies were excluded from absences for women. 
Figure 5. Absence Rate by Job Category and Gender

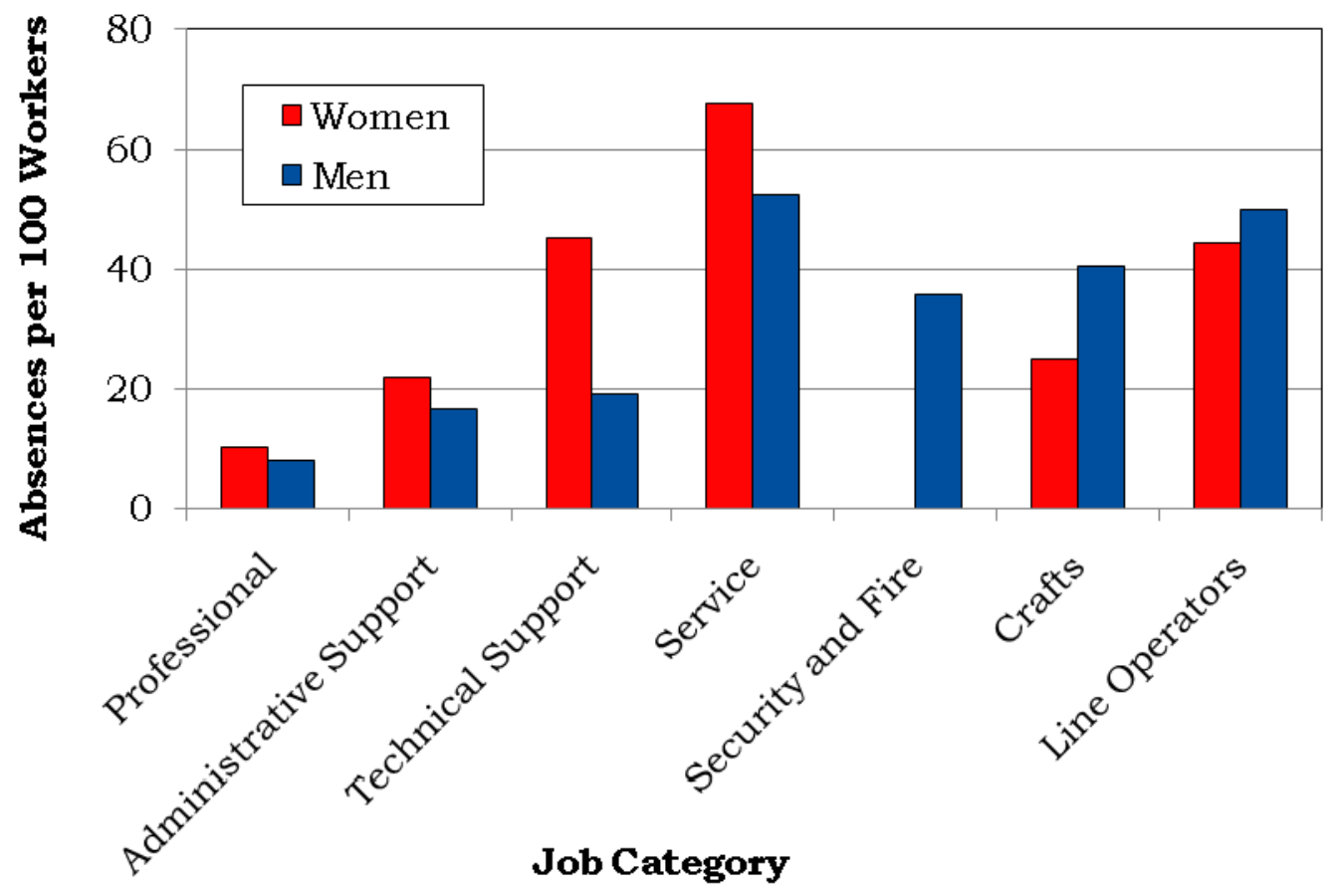

Figure 6. Average Duration of Absence by Job Category and Gender

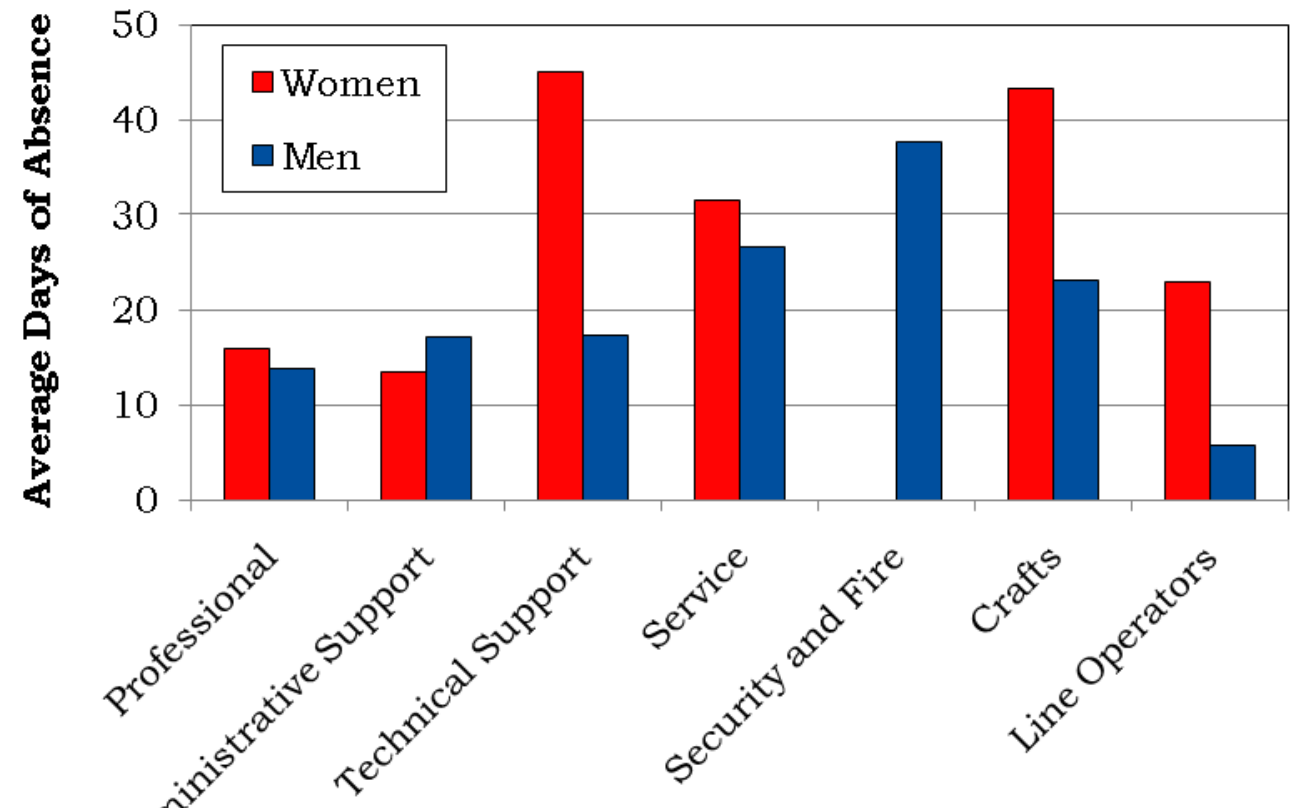

Job Category 


\section{Diagnostic Categories}

Figure 7. Number of Diagnoses and Lost Calendar Days by Diagnostic Category (Categorized by ICD-9-CM) and Gender

\begin{tabular}{|c|c|c|c|c|}
\hline \multirow[b]{2}{*}{ Diagnostic Category } & \multicolumn{2}{|c|}{ Women } & \multicolumn{2}{|c|}{ Men } \\
\hline & $\begin{array}{l}\text { Number of } \\
\text { Diagnoses }\end{array}$ & $\begin{array}{c}\text { Number } \\
\text { of Lost } \\
\text { Calendar } \\
\text { Days }\end{array}$ & $\begin{array}{l}\text { Number of } \\
\text { Diagnoses }\end{array}$ & $\begin{array}{c}\text { Number } \\
\text { of Lost } \\
\text { Calendar } \\
\text { Days }\end{array}$ \\
\hline Benign Growths & 3 & 132 & 4 & 140 \\
\hline Blood & 0 & 0 & 2 & 75 \\
\hline Cancer & 4 & 210 & 11 & 349 \\
\hline Digestive & 17 & 181 & 43 & 507 \\
\hline Endocrine / Me tabolic & 5 & 57 & 7 & 212 \\
\hline Existing Birth Condition & 3 & 171 & 0 & $\mathbf{0}$ \\
\hline Genitourinary & 10 & 304 & 6 & 82 \\
\hline Heart/Circulatory & 10 & 196 & 19 & 660 \\
\hline Infections/Parasites & 7 & 33 & 10 & 47 \\
\hline Injury & 21 & 460 & 53 & 1,401 \\
\hline Miscarriage & 2 & 9 & NA & NA \\
\hline Musculoskeletal & 28 & 723 & 59 & 1,187 \\
\hline Nervous System & 12 & 405 & 17 & 282 \\
\hline Psychological & 8 & 153 & 28 & 496 \\
\hline Respiratory & 63 & 350 & 88 & 515 \\
\hline Skin & 1 & 3 & 11 & 116 \\
\hline Unspecified Symptoms & 30 & 242 & 46 & 421 \\
\hline
\end{tabular}

Note: Lost calendar days for each absence are counted more than once when multiple diagnoses occur in different diagnostic categories for the same absence. 


\section{Figure 8. Common Diagnoses Among Female Workers in 2009}

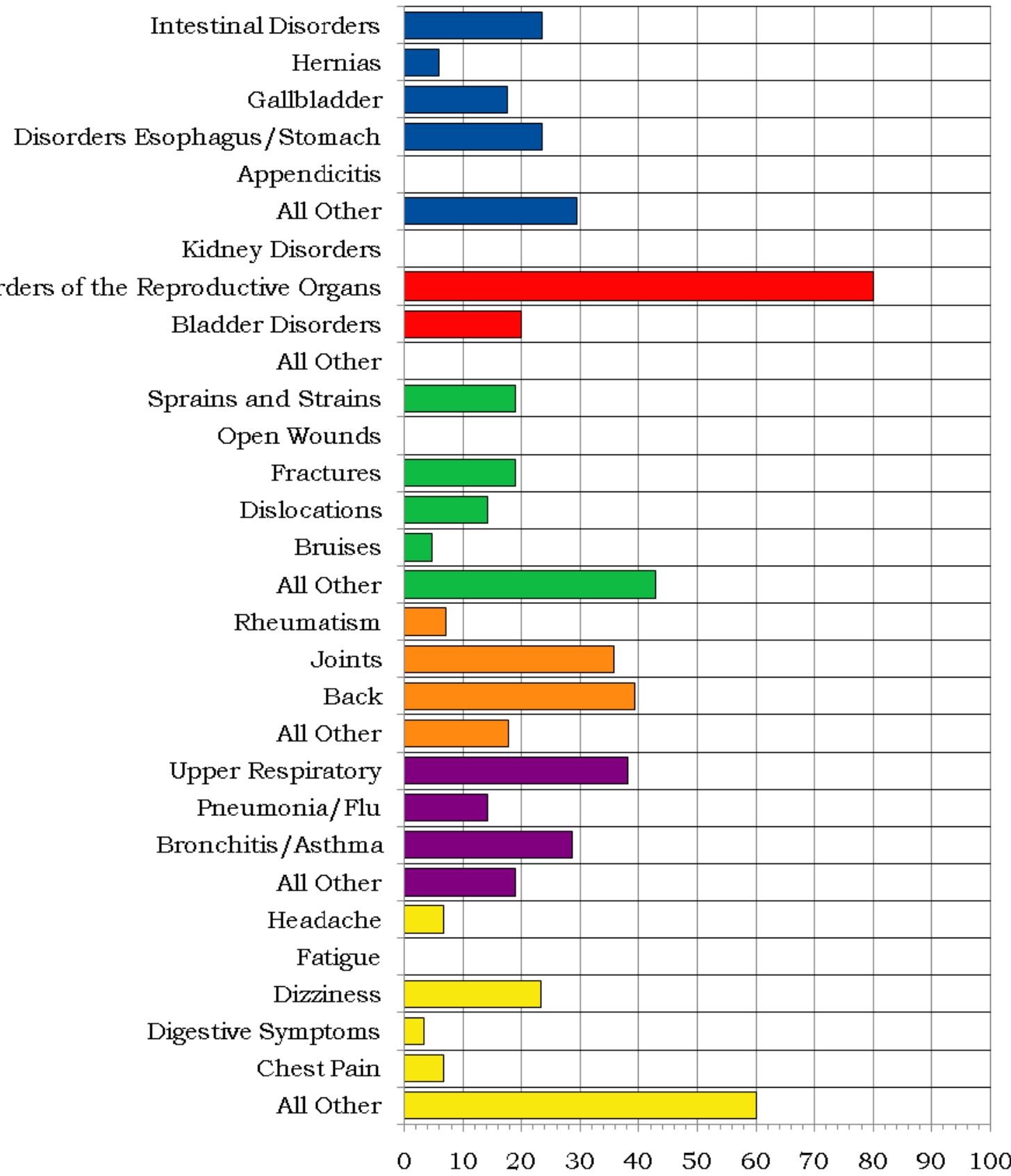

Percent Distribution of Diagnoses Within Diagnostic Category

Dige stive, 17 Diagnoses

Genitourinary, 10 Diagnoses

Injury, 21 Diagnoses
Musculoskeletal, 28 Diagnoses

Respiratory, 63 Diagnoses

Unspecified Symptoms, 30 Diagnoses 
Figure 9. Common Diagnoses Among Male Workers in 2009

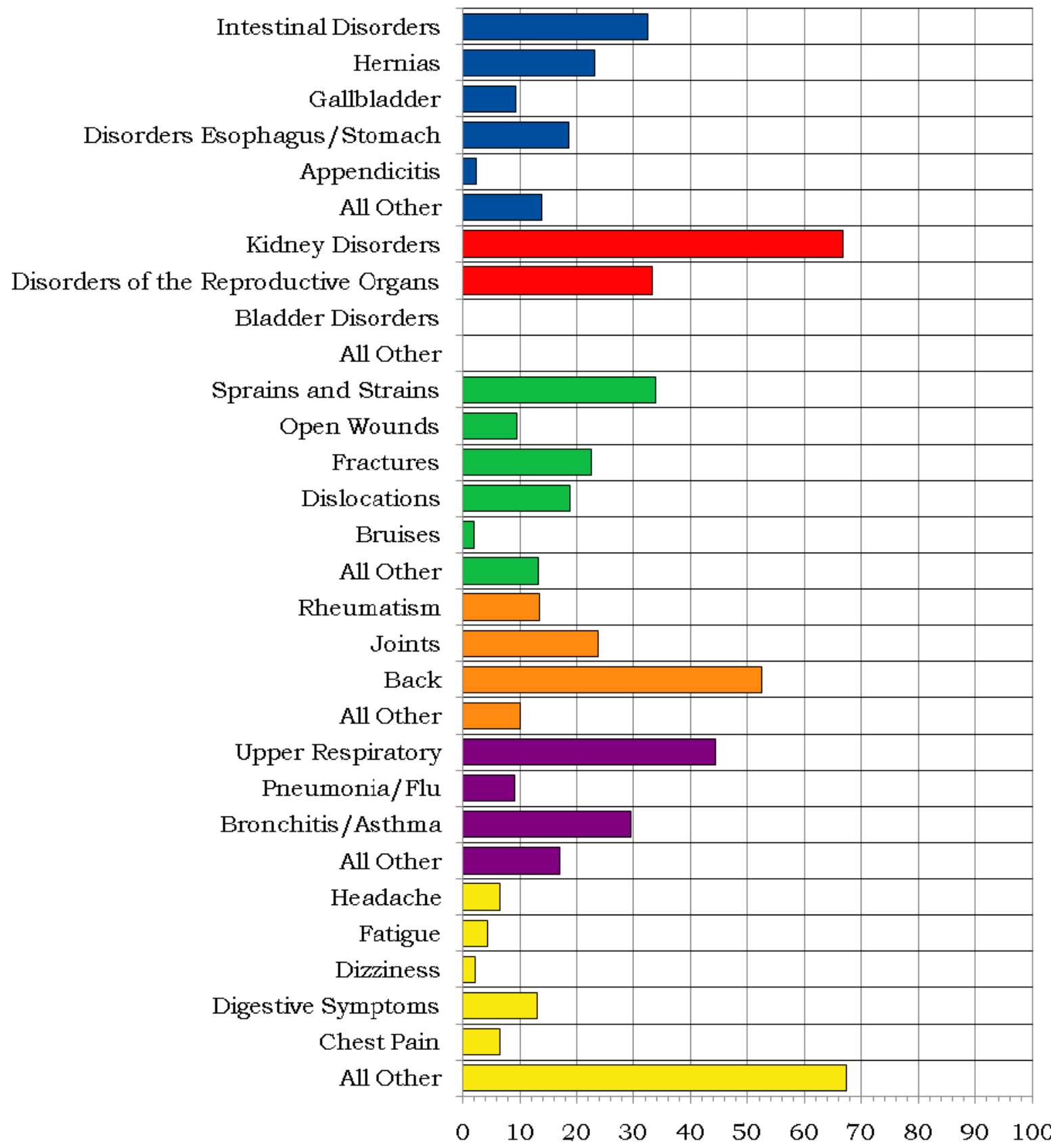

Percent Distribution of Diagnoses Within Diagnostic Category

Digestive, 43 Diagnoses

Genitourinary, 6 Diagnoses

Injury, 53 Diagnoses
Musculoskeletal, 59 Diagnoses

Respiratory, 88 Diagnoses

Unspecified Symptoms, 46 Diagnoses 


\section{Figure 10. Number of Most Frequently Reported Diagnoses by Job Category and Gender}

\begin{tabular}{|c|c|c|c|c|}
\hline Job Category & Men & & Women & \\
\hline & Respiratory & 41 & Respiratory & 23 \\
\hline Professional & Musculoskeletal & 26 & Unspecified Symptoms & 7 \\
\hline & Digestive & 26 & Injury & 6 \\
\hline & Digestive & 3 & Respiratory & 26 \\
\hline & Injury & 2 & M usculoskeletal & 13 \\
\hline Administrative Sunnort & M usculoskeletal & 1 & Unspecified Symptoms & 13 \\
\hline & Unspecified Symptoms & 1 & & \\
\hline & Nervous System & 1 & & \\
\hline & Genitourinary & 1 & & \\
\hline & Respiratory & 9 & M usculoskeletal & 5 \\
\hline & Injury & 8 & Heart/Circulatory & 3 \\
\hline & M usculoskeletal & 6 & Respiratory & 2 \\
\hline Technical Sunport & & & Injury & 2 \\
\hline & & & Digestive & 2 \\
\hline & & & Infections/Parasites & 2 \\
\hline & & & Psychological & 2 \\
\hline & & & Existing Birth Condition & 2 \\
\hline & M usculoskeletal & 10 & Respiratory & 8 \\
\hline Service & Respiratory & 9 & M usculoskeletal & 6 \\
\hline & Injury & 8 & Unspecified Symptoms & 5 \\
\hline & M usculoskeletal & 2 & & 0 \\
\hline Security and Fire & Injury & 2 & & \\
\hline & Unspecified Symptoms & 2 & & \\
\hline & Respiratory & 26 & Respiratory & 2 \\
\hline Crafts & Unspecified Symptoms & 18 & Genitourinary & 2 \\
\hline & Injury & 14 & M usculoskeletal & 1 \\
\hline & Respiratory & 2 & Unspecified Symptoms & 5 \\
\hline & M usculoskeletal & 1 & Respiratory & 2 \\
\hline & Injury & 1 & & \\
\hline Line Operators & Unspecified Symptoms & 1 & & \\
\hline & Nervous System & 1 & & \\
\hline & Heart/Circulatory & 1 & & \\
\hline & Infections/Parasites & 1 & & \\
\hline & Skin & 1 & & \\
\hline
\end{tabular}




\section{Rates of Disease Occurrence}

Figure 11. Rates for All Illnesses and Injuries Combined by Job Category, Gender, and Age

\begin{tabular}{|c|c|c|c|c|}
\hline \multirow{2}{*}{$\begin{array}{c}\text { All Illnesses \& } \\
\text { Injuries Combined }\end{array}$} & \multicolumn{4}{|c|}{ Rate per 1,000} \\
\hline & Job Category & Age & Men & Women \\
\hline & \multirow{2}{*}{ Professional } & $<50$ & 89 & 59 \\
\hline & & $50+$ & 139 & 215 \\
\hline & \multirow{2}{*}{ Administrative Support } & $<50$ & 233 & 400 \\
\hline & & $50+$ & 111 & 244 \\
\hline & \multirow{2}{*}{ Technical Support } & $<50$ & 205 & 600 \\
\hline & & $50+$ & 318 & 818 \\
\hline & \multirow{2}{*}{ Service } & $<50$ & 914 & 750 \\
\hline & & $50+$ & 786 & 1,333 \\
\hline & \multirow{2}{*}{ Security and Fire } & $<50$ & 500 & 0 \\
\hline & & $50+$ & 750 & 0 \\
\hline & \multirow{2}{*}{ Crafts } & $<50$ & 403 & 333 \\
\hline & & $50+$ & 750 & 444 \\
\hline & \multirow{2}{*}{ Line Operators } & $<50$ & 375 & 0 \\
\hline & & $50+$ & 1,000 & 1,000 \\
\hline
\end{tabular}

Figure 12. Rates for Selected Diagnostic Categories by Job Category, Gender, and Age

\begin{tabular}{|c|c|c|c|c|}
\hline \multirow{2}{*}{ Cancer } & \multicolumn{4}{|c|}{ Rate per 1,000} \\
\hline & Job Category & Age & Men & Women \\
\hline & Professional & $<50$ & 1 & 0 \\
\hline & & $50+$ & 1 & 19 \\
\hline & Administrative Sunnort & $<50$ & 0 & 0 \\
\hline & & $50+$ & 0 & 0 \\
\hline & Technical Support & $<50$ & 0 & 0 \\
\hline & & $50+$ & 35 & 0 \\
\hline & Service & $<50$ & 57 & 0 \\
\hline & 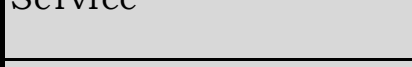 & $50+$ & 0 & 0 \\
\hline & Security and Fire & $<50$ & 0 & 0 \\
\hline & Necuity anturite & $50+$ & 0 & 0 \\
\hline & Crafts & $<50$ & 0 & 0 \\
\hline & & $50+$ & 34 & 0 \\
\hline & ne Opera & $<50$ & 0 & 0 \\
\hline & & $50+$ & 0 & 0 \\
\hline
\end{tabular}


Figure 12. Rates for Selected Diagnostic Categories by Job Category, Gender, and Age (Continued)

\begin{tabular}{|l|l|c|c|c|}
\hline \multirow{2}{*}{ Heart/Circulatory } & \multicolumn{4}{|c|}{ Rate per 1,000 } \\
\cline { 2 - 5 } & \multicolumn{1}{|c|}{ Job Category } & Age & M en & W omen \\
\cline { 2 - 5 } & Profe ssional & $<50$ & $\mathbf{4}$ & $\mathbf{4}$ \\
\cline { 2 - 5 } & $50+$ & $\mathbf{4}$ & $\mathbf{5}$ \\
\hline \multirow{2}{*}{ Administrative Support } & $<50$ & $\mathbf{0}$ & $\mathbf{0}$ \\
\cline { 2 - 5 } & $50+$ & $\mathbf{0}$ & $\mathbf{1 9}$ \\
\hline \multirow{3}{*}{ Technical Support } & $<50$ & $\mathbf{1 2}$ & $\mathbf{5 0}$ \\
\cline { 2 - 5 } & $50+$ & $\mathbf{0}$ & $\mathbf{1 8 2}$ \\
\cline { 2 - 5 } & \multirow{2}{*}{ Service } & $<50$ & $\mathbf{0}$ & $\mathbf{6 3}$ \\
\cline { 2 - 5 } & $50+$ & $\mathbf{7 1}$ & $\mathbf{6 7}$ \\
\cline { 2 - 5 } & \multirow{2}{*}{ Security and Fire } & $<50$ & $\mathbf{0}$ & $\mathbf{0}$ \\
\cline { 2 - 5 } & $50+$ & $\mathbf{0}$ & $\mathbf{0}$ \\
\cline { 2 - 5 } & \multirow{2}{*}{ Crafts } & $<50$ & $\mathbf{1 5}$ & $\mathbf{0}$ \\
\cline { 2 - 5 } & $50+$ & $\mathbf{6 9}$ & $\mathbf{0}$ \\
\cline { 2 - 5 } & \multirow{2}{*}{ Line Operators } & $<50$ & $\mathbf{0}$ & $\mathbf{0}$ \\
\cline { 2 - 5 } & $50+$ & $\mathbf{1 6 7}$ & $\mathbf{0}$ \\
\hline
\end{tabular}

\begin{tabular}{|c|c|c|c|c|}
\hline \multirow{2}{*}{ Respiratory } & \multicolumn{4}{|c|}{ Rate per 1,000} \\
\hline & Job Category & Age & Men & Women \\
\hline & \multirow{2}{*}{ Professional } & $<50$ & 30 & 24 \\
\hline & & $50+$ & 26 & 81 \\
\hline & \multirow{2}{*}{ Administrative Support } & $<50$ & 0 & 127 \\
\hline & & $50+$ & 0 & 44 \\
\hline & \multirow{2}{*}{ Technical Support } & $<50$ & 36 & 0 \\
\hline & & $50+$ & 71 & 182 \\
\hline & \multirow{2}{*}{ Service } & $<50$ & 171 & 125 \\
\hline & & $50+$ & 107 & 400 \\
\hline & \multirow{2}{*}{ Security and Fire } & $<50$ & 100 & 0 \\
\hline & & $50+$ & 0 & 0 \\
\hline & \multirow{2}{*}{ Crafts } & $<50$ & 134 & 0 \\
\hline & & $50+$ & 147 & 222 \\
\hline & \multirow{2}{*}{ Line Operators } & $<50$ & 125 & 0 \\
\hline & & $50+$ & 167 & 286 \\
\hline
\end{tabular}


Figure 12. Rates for Selected Diagnostic Categories by Job Category, Gender, and Age (Continued)

\begin{tabular}{|c|c|c|c|c|}
\hline \multirow{2}{*}{ Injury } & \multicolumn{4}{|c|}{ Rate per 1,000} \\
\hline & Job Category & Age & Men & Women \\
\hline & \multirow{2}{*}{ Professional } & $<50$ & 7 & 0 \\
\hline & & $50+$ & 18 & 29 \\
\hline & \multirow{2}{*}{ Administrative Support } & $<50$ & 67 & 53 \\
\hline & & $50+$ & 0 & 6 \\
\hline & \multirow{2}{*}{ Technical Support } & $<50$ & 60 & 100 \\
\hline & & $50+$ & 35 & $\mathbf{0}$ \\
\hline & \multirow{2}{*}{ Service } & $<50$ & 143 & 63 \\
\hline & & $50+$ & 107 & 200 \\
\hline & \multirow{2}{*}{ Security and Fire } & $<50$ & 0 & 0 \\
\hline & & $50+$ & 500 & 0 \\
\hline & \multirow{2}{*}{ Crafts } & $<50$ & 45 & 0 \\
\hline & & $50+$ & 95 & 0 \\
\hline & \multirow{2}{*}{ Line Operators } & $<50$ & 0 & 0 \\
\hline & & $50+$ & 167 & 0 \\
\hline
\end{tabular}




\section{Sentinel Health Events for Occupations (SHEOs)}

An occupational sentinel health event (SHEO) is a disease, disability, or death that is likely to be occupationally related. Although sentinel health events may indicate an occupational exposure, many may result from nonoccupational exposures. Sentinel health events are therefore assessed in two categories:

Definite Sentinel Health Events: Diseases that are unlikely to occur in the absence of an occupational exposure (e.g., asbestosis).

Possible Sentinel Health Events: Diseases that may be occupational but can also occur in the absence of an occupational exposure (e.g., lung cancer or carpal tunnel syndrome).

Figure 13. Characteristics of SHEOs by Gender

\begin{tabular}{|l|c|c|c|c|}
\hline \multirow{2}{*}{} & \multicolumn{2}{|c|}{$\begin{array}{c}\text { Total Number of } \\
\text { SHEO Diagnoses }\end{array}$} & \multicolumn{2}{c|}{$\begin{array}{c}\text { Total Number of } \\
\text { Days Absent }\end{array}$} \\
\cline { 2 - 5 } & Men & Women & Men & W omen \\
\hline Definite & 0 & 1 & 0 & 110 \\
\hline Possible & 2 & 4 & 9 & 352 \\
\hline Total & 2 & 5 & 9 & 462 \\
\hline
\end{tabular}

Figure 14. SHEO Diagnoses by Gender

\begin{tabular}{|l|c|c|}
\hline \multirow{2}{*}{ Diagnoses } & \multicolumn{2}{c|}{ Gender } \\
\cline { 2 - 3 } & Women & M en \\
\hline Carpal Tunnel Syndrome & 4 & 0 \\
\hline Injuries & 1 & 0 \\
\hline Other Conditions & 0 & 2 \\
\hline
\end{tabular}


Occupational Safety and Health Administration (OSHA)-Recordable Events

Figure 15. OSHA-Recordable Events by Gender and Age

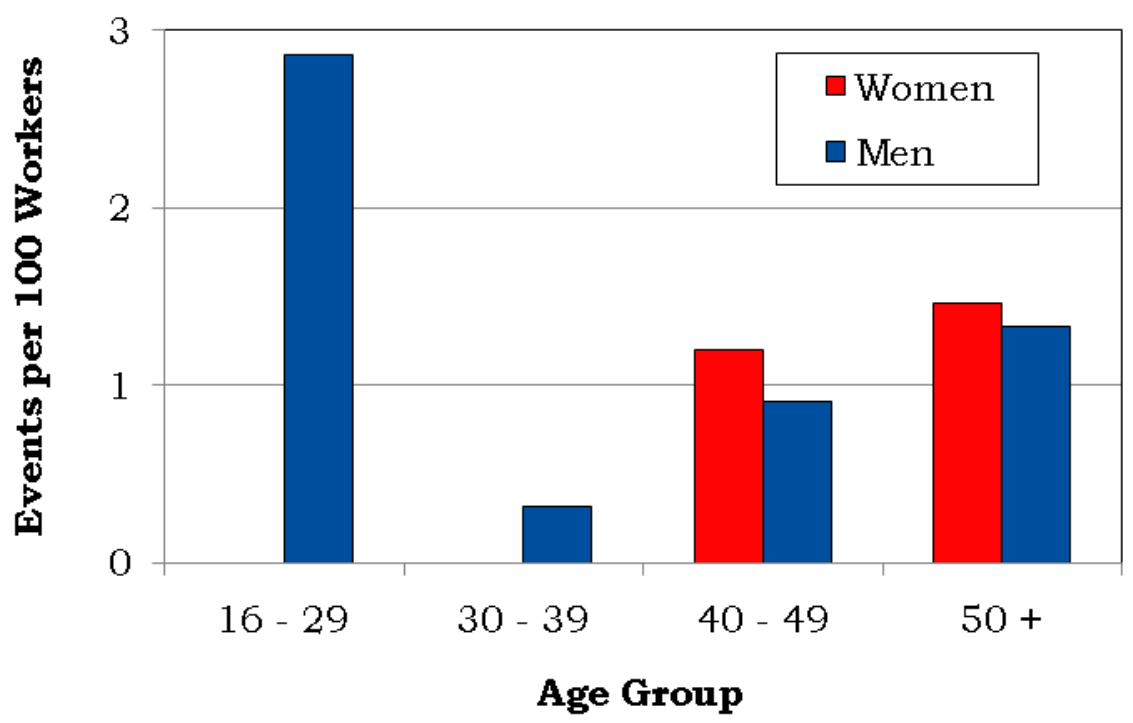

Figure 16. OSHA-Recordable Events by Job Category and Gender

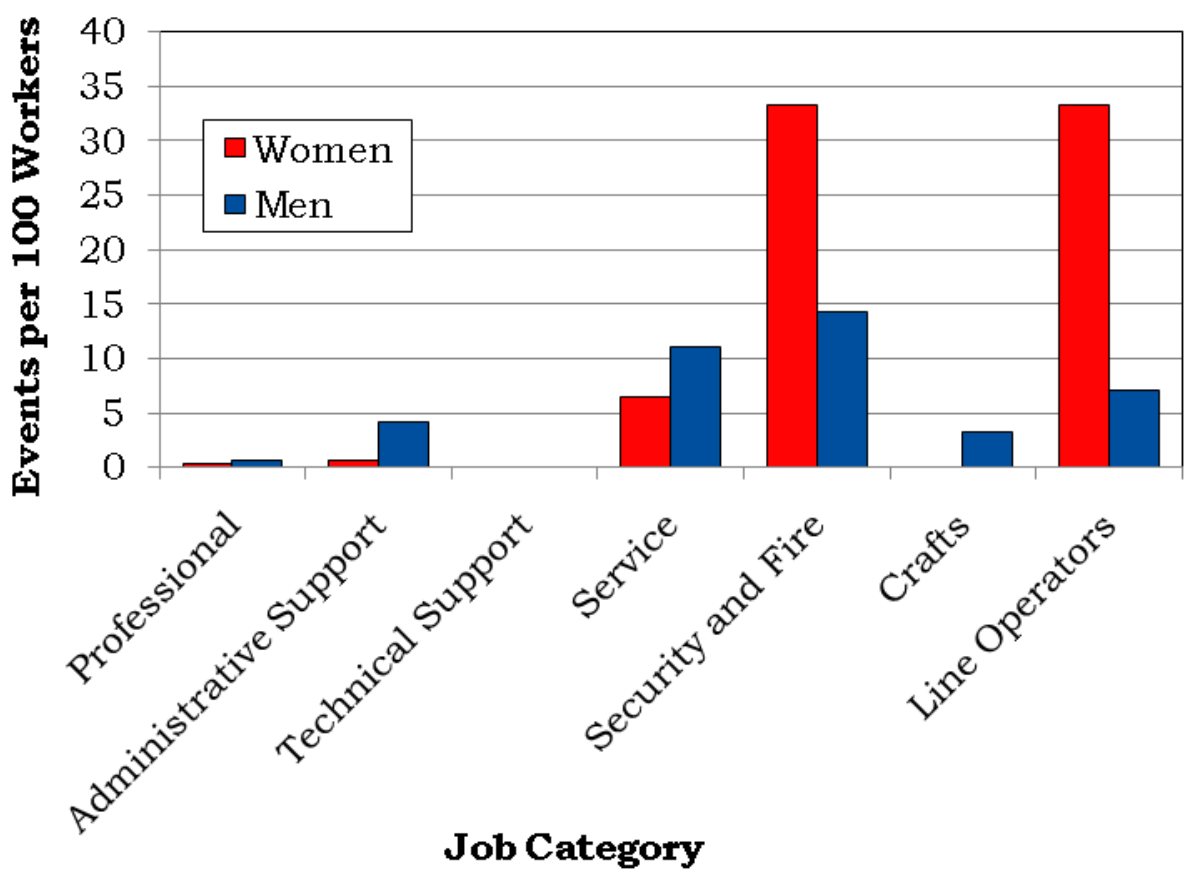




\section{Diagnostic and Accident Categories for OSHA-Recordable Events}

Figure 17. OSHA-Recordable Diagnoses by Diagnostic Category and Gender

\begin{tabular}{|l|c|c|}
\hline \multirow{2}{*}{ Diagnostic Category } & \multicolumn{2}{c|}{ Gender } \\
\cline { 2 - 3 } & Women & M en \\
\hline Musculoskeletal & $\mathbf{4}$ & $\mathbf{6}$ \\
\hline Nervous System & $\mathbf{1}$ & $\mathbf{0}$ \\
\hline Respiratory & $\mathbf{1}$ & $\mathbf{0}$ \\
\hline Unspecified Symptoms & $\mathbf{0}$ & $\mathbf{1}$ \\
\hline Injury & $\mathbf{8}$ & $\mathbf{2 2}$ \\
\hline Fractures - Upper Limb & $\mathbf{0}$ & $\mathbf{2}$ \\
\hline Fractures - Lower Limb & $\mathbf{1}$ & $\mathbf{1}$ \\
\hline Back Sprains \& Strains & $\mathbf{1}$ & $\mathbf{0}$ \\
\hline Other Sprains \& Strains & $\mathbf{2}$ & $\mathbf{8}$ \\
\hline Intracranial Injuries & $\mathbf{0}$ & $\mathbf{1}$ \\
\hline Open Wounds - Upper Limb & $\mathbf{0}$ & $\mathbf{5}$ \\
\hline Bruises & $\mathbf{0}$ & $\mathbf{1}$ \\
\hline Foreign Bodies Entering Orifice & $\mathbf{0}$ & $\mathbf{1}$ \\
\hline Unspecified Injuries & $\mathbf{3}$ & $\mathbf{3}$ \\
\hline Adverse Reactions to Non-Medical Substances & $\mathbf{1}$ & $\mathbf{0}$ \\
\hline
\end{tabular}

Figure 18. OSHA-Recordable Accidents by Type and Gender

\begin{tabular}{|l|c|c|}
\hline \multirow{2}{*}{ Accident Category } & \multicolumn{2}{c|}{ Gender } \\
\cline { 2 - 3 } & Women & Men \\
\cline { 2 - 3 } & $\begin{array}{c}\text { Number of } \\
\text { Accidents }\end{array}$ & $\begin{array}{c}\text { Number of } \\
\text { Accidents }\end{array}$ \\
\hline Falls & $\mathbf{2}$ & $\mathbf{3}$ \\
\hline Fire & $\mathbf{1}$ & $\mathbf{0}$ \\
\hline Natural/Environmental Factors & $\mathbf{1}$ & $\mathbf{0}$ \\
\hline Submersion/Suffocation/Foreign Bodies & $\mathbf{0}$ & $\mathbf{1}$ \\
\hline Other Accidents & $\mathbf{5}$ & $\mathbf{1 8}$ \\
\hline Struck by an Object & $\mathbf{0}$ & $\mathbf{4}$ \\
\hline Machinery & $\mathbf{0}$ & $\mathbf{1}$ \\
\hline Cutting/Piercing Instrument/Object & $\mathbf{0}$ & $\mathbf{4}$ \\
\hline Overexertion/Strenuous Movements & $\mathbf{5}$ & $\mathbf{8}$ \\
\hline Vibration & $\mathbf{0}$ & $\mathbf{1}$ \\
\hline Total & $\mathbf{9}$ & $\mathbf{2 2}$ \\
\hline
\end{tabular}




\section{Rates of OSHA-Recordable Events}

Figure 19. OSHA-Recordable Rates by Age and Job Categories Among Women, All Diagnoses Combined

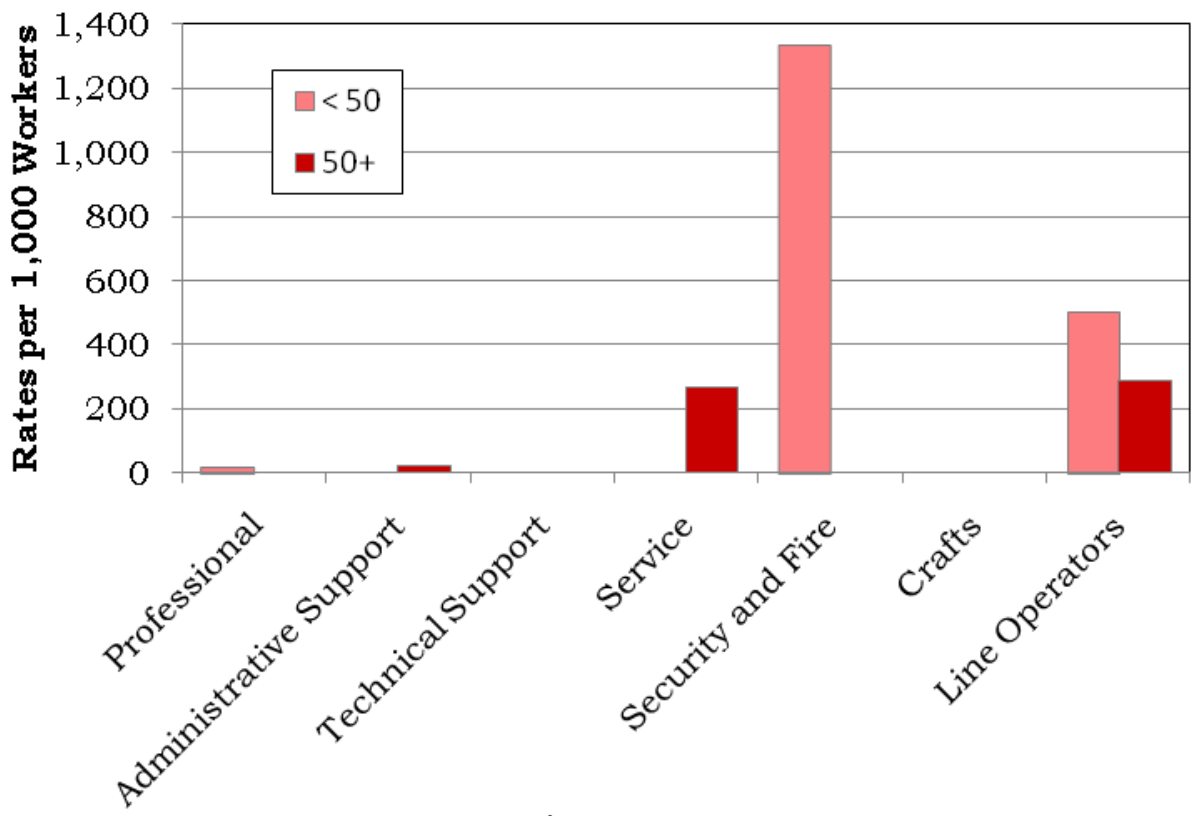

Job Category

Figure 20. OSHA-Recordable Rates by Age and Job Categories Among Men, All Diagnoses Combined

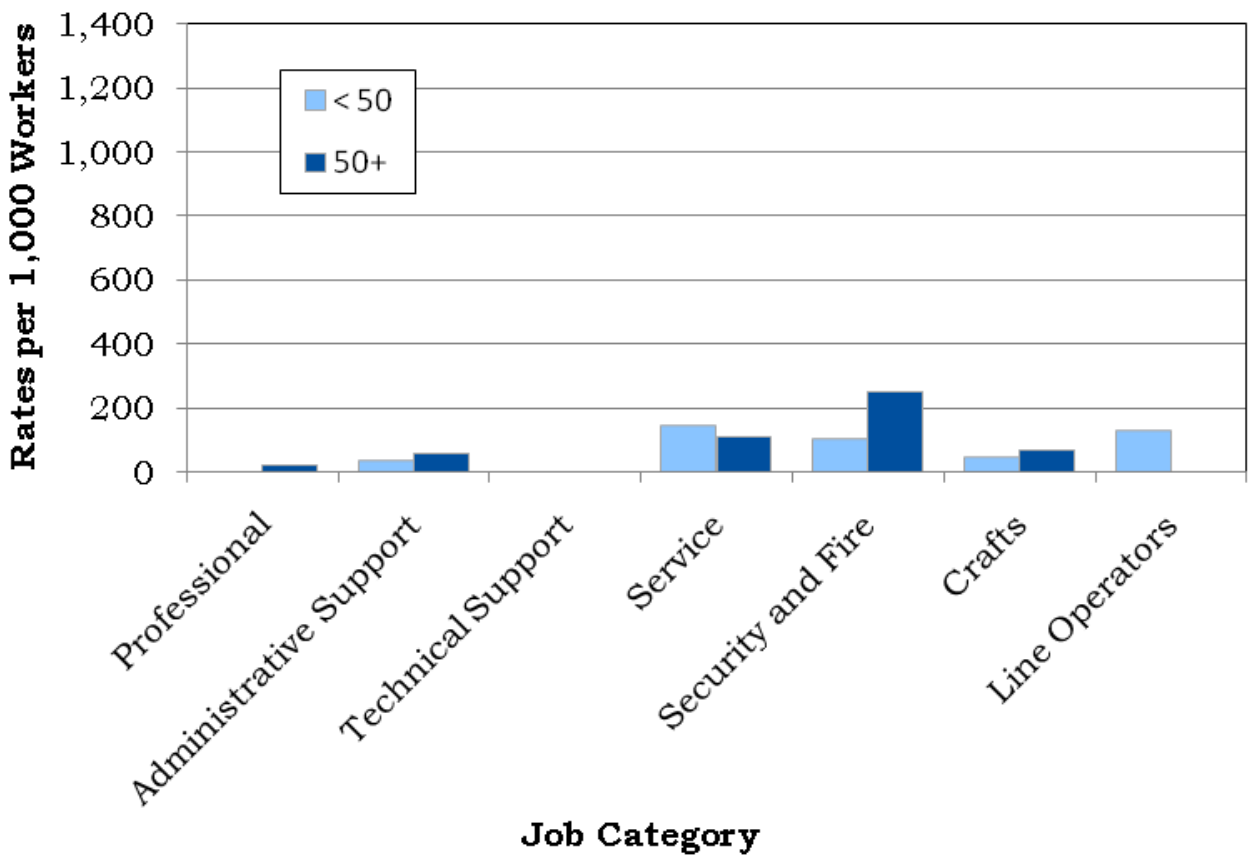




\section{Appendices}


Argonne National Laboratory 2009

Absence Data

Appendix A. Work Force by Gender, Age, and Job Category

\begin{tabular}{|c|c|c|c|c|c|c|c|c|c|c|c|}
\hline \multirow{3}{*}{ Job Category } & \multicolumn{5}{|c|}{ Women } & \multicolumn{5}{|c|}{ Men } & \multirow[b]{3}{*}{ TOTAL } \\
\hline & \multicolumn{4}{|c|}{ Age Group } & \multirow[b]{2}{*}{ TOTAL } & \multicolumn{4}{|c|}{ Age Group } & \multirow[b]{2}{*}{ TOTAL } & \\
\hline & $16-29$ & 30 - 39 & $40-49$ & $50+$ & & $16-29$ & 30 - 39 & $40-49$ & $50+$ & & \\
\hline Professional & 28 & 84 & 142 & 209 & 463 & 62 & 251 & 425 & 717 & 1,455 & 1,918 \\
\hline Administrative Support & 29 & 40 & 81 & 160 & 310 & 11 & 9 & 10 & 18 & 48 & 358 \\
\hline Technical Support & 5 & 2 & 13 & 11 & 31 & 11 & 19 & 53 & 85 & 168 & 199 \\
\hline Service & 1 & 4 & 11 & 15 & 31 & 8 & 11 & 16 & 28 & 63 & 94 \\
\hline Security and Fire & 1 & 2 & 0 & 0 & 3 & 2 & 3 & 5 & 4 & 14 & 17 \\
\hline Crafts & 0 & 0 & 3 & 9 & 12 & 6 & 19 & 42 & 116 & 183 & 195 \\
\hline Line Operators & 0 & 1 & 1 & 7 & 9 & 5 & 2 & 1 & 6 & 14 & 23 \\
\hline TOTAL & 64 & 133 & 251 & 411 & 859 & 105 & 314 & 552 & 974 & 1,945 & 2,804 \\
\hline
\end{tabular}

Appendix B. Age Distribution of the Work Force by Gender

\begin{tabular}{|l|r|r|r|r|r|r|r|r|}
\hline \multirow{3}{*}{ Year } & \multicolumn{4}{|c|}{ Women } & \multicolumn{4}{c|}{ Men } \\
\cline { 2 - 9 } & \multicolumn{4}{|c|}{ Percent Distribution by Age Group } & \multicolumn{3}{|c|}{ Percent Distribution by Age Group } \\
\cline { 2 - 9 } & $\mathbf{1 6}-\mathbf{2 9}$ & $\mathbf{3 0}-\mathbf{3 9}$ & $\mathbf{4 0}-\mathbf{4 9}$ & $\mathbf{5 0}+$ & $\mathbf{1 6}-\mathbf{2 9}$ & $\mathbf{3 0}-\mathbf{3 9}$ & $\mathbf{4 0}-\mathbf{4 9}$ & $\mathbf{5 0}+$ \\
\hline $\mathbf{2 0 0 9}$ & 7.45 & 15.48 & 29.22 & 47.85 & 5.40 & 16.14 & 28.38 & 50.08 \\
\hline
\end{tabular}


Appendix C. Total Number of Workers Who Reported at Least One Absence by Gender, Age, and Job Category*

\begin{tabular}{|c|c|c|c|c|c|c|c|c|c|c|c|}
\hline \multirow{3}{*}{ Job Category } & \multicolumn{5}{|c|}{ Women } & \multicolumn{5}{|c|}{ Men } & \multirow[b]{3}{*}{ TOTAL } \\
\hline & \multicolumn{4}{|c|}{ Age Group } & \multirow[b]{2}{*}{ TOTAL } & \multicolumn{4}{|c|}{ Age Group } & \multirow[b]{2}{*}{ TOTAL } & \\
\hline & $16-29$ & 30 - 39 & $40-49$ & $50+$ & & $16-29$ & 30 - 39 & $40-49$ & $50+$ & & \\
\hline Professional & 2 & 3 & 9 & 27 & 41 & 4 & 11 & 28 & 53 & 96 & 137 \\
\hline Administrative Support & 8 & 3 & 13 & 26 & 50 & 1 & 3 & 2 & 1 & 7 & 57 \\
\hline Technical Support & 1 & 1 & 3 & 5 & 10 & 3 & 1 & 10 & 15 & 29 & 39 \\
\hline Service & 1 & 2 & 4 & 9 & 16 & 2 & 6 & 5 & 10 & 23 & 39 \\
\hline Security and Fire & 0 & 0 & 0 & 0 & 0 & 1 & 1 & 1 & 1 & 4 & 4 \\
\hline Crafts & 0 & 0 & 1 & 2 & 3 & 2 & 4 & 10 & 35 & 51 & 54 \\
\hline Line Operators & 0 & 0 & 0 & 2 & 2 & 2 & 0 & 0 & 3 & 5 & 7 \\
\hline TOTAL & 12 & 9 & 30 & 71 & 122 & 15 & 26 & 56 & 118 & 215 & 337 \\
\hline
\end{tabular}

*Only those job categories and gender/age combinations with at least one absence appear in this table.

Appendix D. Total Number of Absences by Gender, Age, and Job Category*

\begin{tabular}{|c|c|c|c|c|c|c|c|c|c|c|c|}
\hline \multirow{3}{*}{ Job Category } & \multicolumn{5}{|c|}{ Women } & \multicolumn{5}{|c|}{ Men } & \multirow[b]{3}{*}{ TOTAL } \\
\hline & \multicolumn{4}{|c|}{ Age Group } & \multirow[b]{2}{*}{ TOTAL } & \multicolumn{4}{|c|}{ Age Group } & \multirow[b]{2}{*}{ TOTAL } & \\
\hline & $16-29$ & 30 - 39 & $40-49$ & $50+$ & & $16-29$ & 30 - 39 & $40-49$ & $50+$ & & \\
\hline Professional & 2 & 3 & 9 & 33 & 47 & 5 & 11 & 31 & 71 & 118 & 165 \\
\hline Administrative Support & 12 & 3 & 24 & 29 & 68 & 1 & 4 & 2 & 1 & 8 & 76 \\
\hline Technical Support & 1 & 1 & 6 & 6 & 14 & 4 & 1 & 10 & 17 & 32 & 46 \\
\hline Service & 1 & 2 & 5 & 13 & 21 & 2 & 9 & 8 & 14 & 33 & 54 \\
\hline Security and Fire & 0 & 0 & 0 & 0 & 0 & 2 & 1 & 1 & 1 & 5 & 5 \\
\hline Crafts & 0 & 0 & 1 & 2 & 3 & 2 & 4 & 13 & 55 & 74 & 77 \\
\hline Line Operators & 0 & 0 & 0 & 4 & 4 & 3 & 0 & 0 & 4 & 7 & 11 \\
\hline TOTAL & 16 & 9 & 45 & 87 & 157 & 19 & 30 & 65 & 163 & 277 & 434 \\
\hline
\end{tabular}

*Only those job categories and gender/age combinations with at least one absence appear in this table. 
Argonne National Laboratory 2009

Absence Data

Appendix E. Distribution of the Number of Calendar Days Missed per Absence by Gender and Age*

\begin{tabular}{|c|c|c|c|c|c|c|c|c|c|c|c|}
\hline \multirow{3}{*}{$\begin{array}{c}\text { Number of } \\
\text { Calendar Days }\end{array}$} & \multicolumn{5}{|c|}{ Women } & \multicolumn{5}{|c|}{ Men } & \multirow[b]{3}{*}{ TOTAL } \\
\hline & \multicolumn{4}{|c|}{ Age Group } & \multirow[b]{2}{*}{ TOTAL } & \multicolumn{4}{|c|}{ Age Group } & \multirow[b]{2}{*}{ TOTAL } & \\
\hline & $16-29$ & 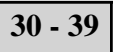 & 40 - 49 & $50+$ & & $16-29$ & $30-39$ & $40-49$ & $50+$ & & \\
\hline$<15$ & 12 & 7 & 33 & 55 & 107 & 14 & 22 & 49 & 106 & 191 & 298 \\
\hline $15-28$ & 3 & 1 & 5 & 11 & 20 & 3 & 2 & 6 & 19 & 30 & 50 \\
\hline $29-42$ & 1 & 1 & 1 & 8 & 11 & 0 & 2 & 1 & 8 & 11 & 22 \\
\hline $43-56$ & 0 & 0 & 2 & 0 & 2 & 1 & 2 & 4 & 11 & 18 & 20 \\
\hline $57-91$ & 0 & 0 & 3 & 7 & 10 & 1 & 1 & 3 & 13 & 18 & 28 \\
\hline $92-182$ & 0 & 0 & 1 & 5 & 6 & 0 & 1 & 2 & 5 & 8 & 14 \\
\hline $183+$ & 0 & 0 & 0 & 1 & 1 & 0 & 0 & 0 & 1 & 1 & 2 \\
\hline TOTAL & 16 & 9 & 45 & 87 & 157 & 19 & 30 & 65 & 163 & 277 & 434 \\
\hline
\end{tabular}

*Only those gender/age combinations with at least one absence appear in this table. 
Argonne National Laboratory 2009

Absence Data

Appendix F. Distribution of the Number of Calendar Days Missed per Absence by Gender and Job Category*

Women

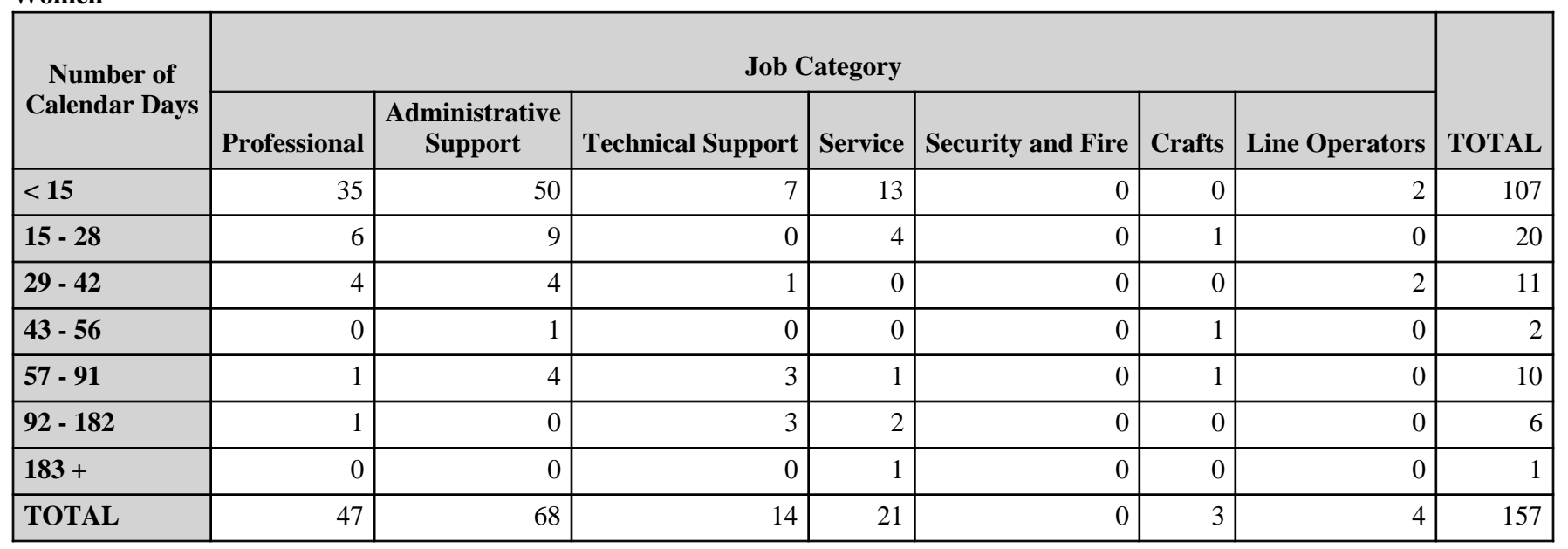

Men

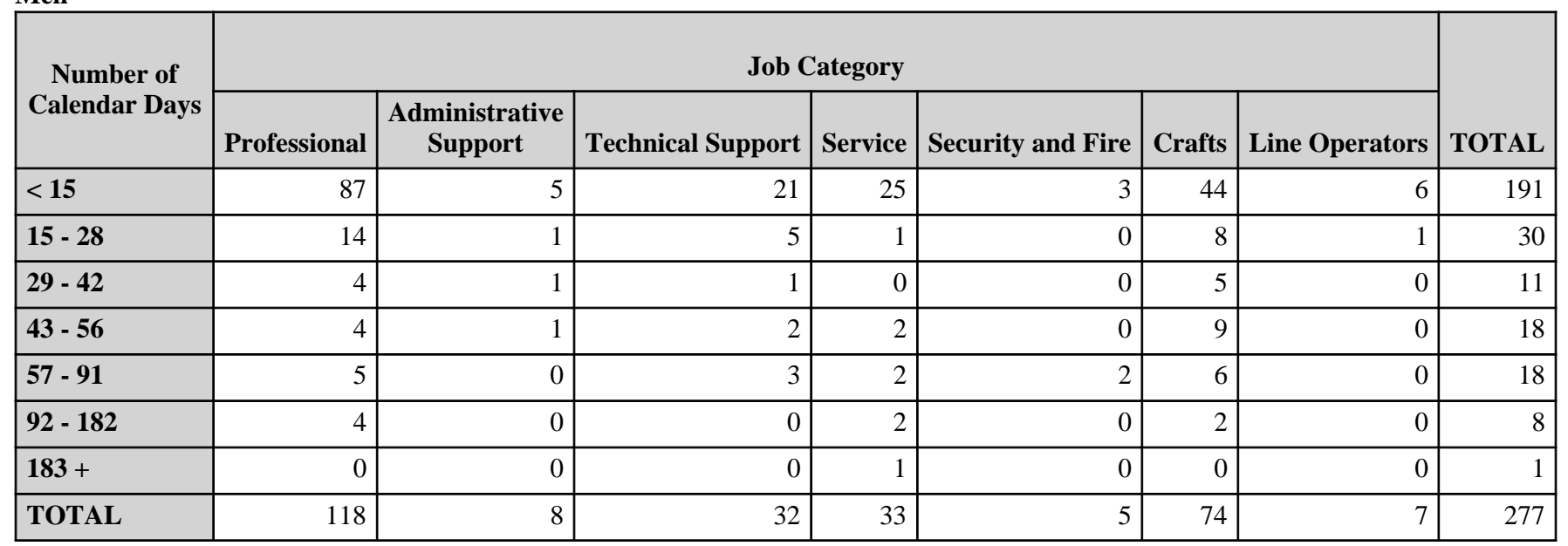

*Only those gender/job category combinations with at least one absence appear in this table. 
Argonne National Laboratory 2009

Absence Data

Appendix G. Number of Diagnoses in Each Diagnostic Category by Gender and Age*

\begin{tabular}{|c|c|c|c|c|c|c|}
\hline & & \multicolumn{5}{|c|}{ Women } \\
\hline & & \multicolumn{4}{|c|}{ Age Group } & \multirow[b]{2}{*}{ TOTAL } \\
\hline & & $16-29$ & $30-39$ & $40-49$ & $\mathbf{5 0 +}$ & \\
\hline Diagnostic Category & ICD-9-CM Code & \multirow[b]{2}{*}{0} & \multirow[b]{2}{*}{0} & \multirow[b]{2}{*}{4} & \multirow[b]{2}{*}{3} & \multirow[b]{2}{*}{7} \\
\hline INFECTIOUS \& PARASITIC DISEASES (DIS) & 001-139 & & & & & \\
\hline -Intestinal Infectious Dis & 001-009 & 0 & 0 & 1 & 0 & 1 \\
\hline -Other Bacterial Dis & 030-041 & 0 & 0 & 1 & 0 & 1 \\
\hline -Polio \& Other Viral CNS Dis & 045-049 & 0 & 0 & 0 & 0 & 0 \\
\hline -Other Viral Dis \& Chlamydiae & 070-079 & 0 & 0 & 2 & 3 & 5 \\
\hline MALIGNANT NEOPLASMS & $\begin{array}{l}140-208 \\
209.0-209.3 \\
230-234\end{array}$ & 0 & 0 & 0 & 4 & 4 \\
\hline -Digestive \& Peritoneal & $150-159$ & 0 & 0 & 0 & 1 & 1 \\
\hline -Bone, Connective Tissue, Skin & $170-173,176$ & 0 & 0 & 0 & 0 & 0 \\
\hline -Breast & 174-175 & 0 & 0 & 0 & 1 & 1 \\
\hline -Genitourinary & 179-189 & 0 & 0 & 0 & 1 & 1 \\
\hline -Other \& Unspecified Sites & $195-199,209.7$ & 0 & 0 & 0 & 1 & 1 \\
\hline BENIGN \& UNCERTAIN NEOPLASMS & $\begin{array}{l}209.4-209.6 \\
210-229,235-239\end{array}$ & 1 & 0 & 1 & 1 & 3 \\
\hline ENDOCRINE/METABOLIC/IMMUNITY & $240-279$ & 1 & 0 & 0 & 4 & 5 \\
\hline -Other Endocrine Gland Dis & $249-259$ & 1 & 0 & 0 & 3 & 4 \\
\hline -Other Metabolic \& Immunity Disorders & $270-279$ & 0 & 0 & 0 & 1 & 1 \\
\hline BLOOD \& BLOOD-FORMING ORGANS & $280-289$ & 0 & 0 & 0 & 0 & 0 \\
\hline MENTAL DISORDERS & 290-319 & 0 & 0 & 8 & 0 & 8 \\
\hline -Psychoses & $290-299$ & 0 & 0 & 0 & 0 & 0 \\
\hline -Non-Psychotic Disorders & $300-302,306-316$ & 0 & 0 & 8 & 0 & 8 \\
\hline -Alcohol Dependence & 303 & 0 & 0 & 0 & 0 & 0 \\
\hline -Drug Dependence & $304-305$ & 0 & 0 & 0 & 0 & 0 \\
\hline NERVOUS SYSTEM (NS) \& SENSE ORGANS & 320-389 & 2 & 1 & 3 & 6 & 12 \\
\hline -Other Disorders of Central NS & $340-349$ & 1 & 1 & 0 & 1 & 3 \\
\hline -Disorders of Peripheral NS & $350-359$ & 0 & 0 & 1 & 4 & 5 \\
\hline -Disorders of Eye & $360-379$ & 0 & 0 & 1 & 1 & 2 \\
\hline -Diseases of Ear \& Mastoid & $380-389$ & 1 & 0 & 1 & 0 & 2 \\
\hline CIRCULATORY SYSTEM & $390-459$ & 1 & 0 & 2 & 7 & 10 \\
\hline -Hypertensive Dis & $401-405$ & 0 & 0 & 0 & 2 & 2 \\
\hline -Ischemic Heart Dis & $410-414$ & 0 & 0 & 0 & 2 & 2 \\
\hline -Dis of Pulmonary Circulation & $415-417$ & 0 & 0 & 0 & 0 & 0 \\
\hline -Other Heart Dis & $420-429$ & 1 & 0 & 1 & 0 & 2 \\
\hline -Cerebrovascular Dis & $430-438$ & 0 & 0 & 1 & 1 & 2 \\
\hline -Dis of Arteries \& Capillaries & $440-449$ & 0 & 0 & 0 & 0 & 0 \\
\hline -Dis of Veins, Lymphatics, Other & 451-459 & 0 & 0 & 0 & 2 & 2 \\
\hline RESPIRATORY SYSTEM & $460-519$ & 5 & 3 & 19 & 36 & 63 \\
\hline
\end{tabular}

(Continued)

*Only those diagnostic categories and gender/age combinations with at least one occurrence appear in this table. 
Argonne National Laboratory 2009

Absence Data

Appendix G. Number of Diagnoses in Each Diagnostic Category by Gender and Age*

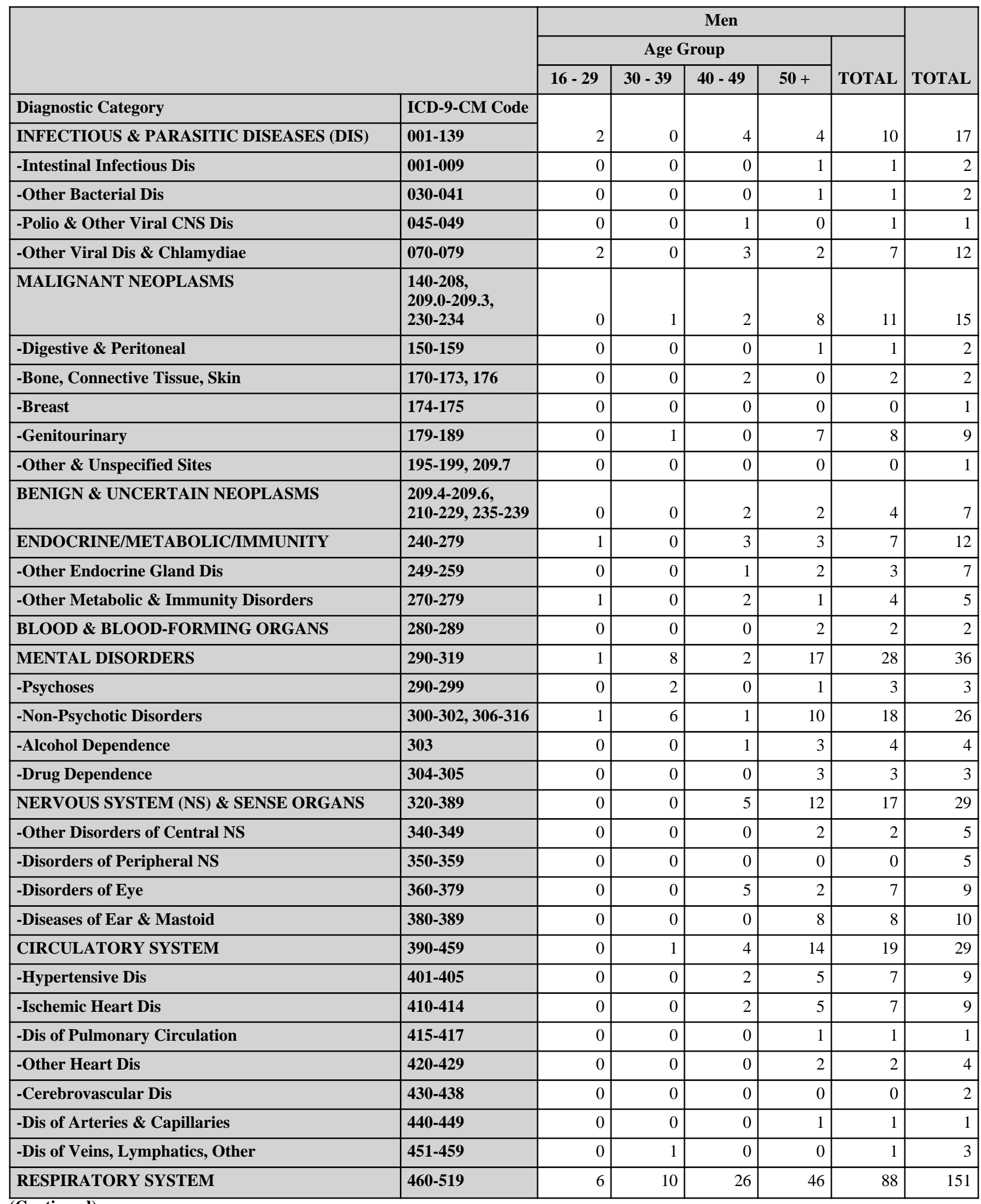

(Continued)

*Only those diagnostic categories and gender/age combinations with at least one occurrence appear in this table. 
Argonne National Laboratory 2009

Absence Data

Appendix G. Number of Diagnoses in Each Diagnostic Category by Gender and Age*

\begin{tabular}{|c|c|c|c|c|c|c|}
\hline & & \multicolumn{5}{|c|}{ Women } \\
\hline & & \multicolumn{4}{|c|}{ Age Group } & \multirow[b]{2}{*}{ TOTAL } \\
\hline & & $16-29$ & $30-39$ & $40-49$ & $50+$ & \\
\hline Diagnostic Category & ICD-9-CM Code & \multirow[b]{2}{*}{1} & \multirow[b]{2}{*}{0} & \multirow[b]{2}{*}{8} & \multirow[b]{2}{*}{9} & \multirow[b]{2}{*}{18} \\
\hline -Acute Respiratory Infections & $460-466$ & & & & & \\
\hline -Other Dis Upper Respiratory Tract & $470-478$ & 2 & 0 & 1 & 3 & 6 \\
\hline -Pneumonia \& Influenza & $480-488$ & 2 & 2 & 6 & 9 & 19 \\
\hline -Chronic Obstructive Dis & $490-496$ & 0 & 1 & 4 & 13 & 18 \\
\hline -Lung Dis from External Agents & $500-508$ & 0 & 0 & 0 & 0 & 0 \\
\hline -Other Respiratory Dis & $510-519$ & 0 & 0 & 0 & 2 & 2 \\
\hline DIGESTIVE SYSTEM & $520-579$ & 1 & 2 & 5 & 9 & 17 \\
\hline -Oral Cavity, Saliva Glands, Jaw & $520-529$ & 0 & 1 & 1 & 3 & 5 \\
\hline -Esophagus, Stomach, Duodenum & $530-538$ & 0 & 0 & 1 & 3 & 4 \\
\hline -Appendicitis & $540-543$ & 0 & 0 & 0 & 0 & 0 \\
\hline -Hernias & $550-553$ & 0 & 1 & 0 & 0 & 1 \\
\hline -Enteritis, Colitis & $555-558$ & 0 & 0 & 1 & 1 & 2 \\
\hline -Other Intestinal Dis & $560-569$ & 0 & 0 & 1 & 1 & 2 \\
\hline -Other Digestive Dis & $570-579$ & 1 & 0 & 1 & 1 & 3 \\
\hline GENITOURINARY SYSTEM & $580-629$ & 2 & 0 & 0 & 8 & 10 \\
\hline -Other Urinary Dis & $590-599$ & 1 & 0 & 0 & 1 & 2 \\
\hline -Male Genital Organ Dis & 600-608 & 0 & 0 & 0 & 0 & 0 \\
\hline -Pelvic Inflammatory Dis & 614-616 & 1 & 0 & 0 & 0 & 1 \\
\hline -Other Female Disorders & $617-629$ & 0 & 0 & 0 & 7 & 7 \\
\hline PREGNANCY \& CHILDBIRTH & $630-679$ & 1 & 1 & 0 & 0 & 2 \\
\hline -Pregnancy with Abortive Outcome & 634-639 & 1 & 1 & 0 & 0 & 2 \\
\hline SKIN AND SUBCUTANEOUS TISSUE & $680-709$ & 0 & 0 & 0 & 1 & 1 \\
\hline -Infections & 680-686 & 0 & 0 & 0 & 1 & 1 \\
\hline -Other Inflammatory Conditions & $690-698$ & 0 & 0 & 0 & 0 & 0 \\
\hline -Other & \begin{tabular}{|l|}
$700-709$ \\
\end{tabular} & 0 & 0 & 0 & 0 & 0 \\
\hline $\begin{array}{l}\text { MUSCULOSKELETAL \& CONNECTIVE } \\
\text { TISSUE }\end{array}$ & $710-739$ & 0 & 1 & 8 & 19 & 28 \\
\hline -Arthropathies & $710-719$ & 0 & 0 & 0 & 10 & 10 \\
\hline -Dorsopathies & $720-724$ & 0 & 0 & 6 & 5 & 11 \\
\hline -Rheumatism, Excluding Back & 725-729 & 0 & 0 & 1 & 1 & 2 \\
\hline -Other Dis \& Acquired Deformities & \begin{tabular}{|l|}
$730-739$ \\
\end{tabular} & 0 & 1 & 1 & 3 & 5 \\
\hline CONGENITAL ANOMALIES & $740-759$ & 0 & 0 & 2 & 1 & 3 \\
\hline $\begin{array}{l}\text { SYMPTOMS, SIGNS, \& ILL-DEFINED } \\
\text { CONDITIONS }\end{array}$ & $780-799$ & 6 & 2 & 7 & 15 & 30 \\
\hline -Symptoms & 780-789 & 5 & 2 & 7 & 14 & 28 \\
\hline -Non-Specific Abnormal Findings & $790-796$ & 1 & 0 & 0 & 1 & 2 \\
\hline INJURY \& POISONING & $800-999$ & 0 & 1 & 10 & 10 & 21 \\
\hline
\end{tabular}

(Continued)

*Only those diagnostic categories and gender/age combinations with at least one occurrence appear in this table. 


\section{Argonne National Laboratory 2009}

\section{Absence Data}

Appendix G. Number of Diagnoses in Each Diagnostic Category by Gender and Age*

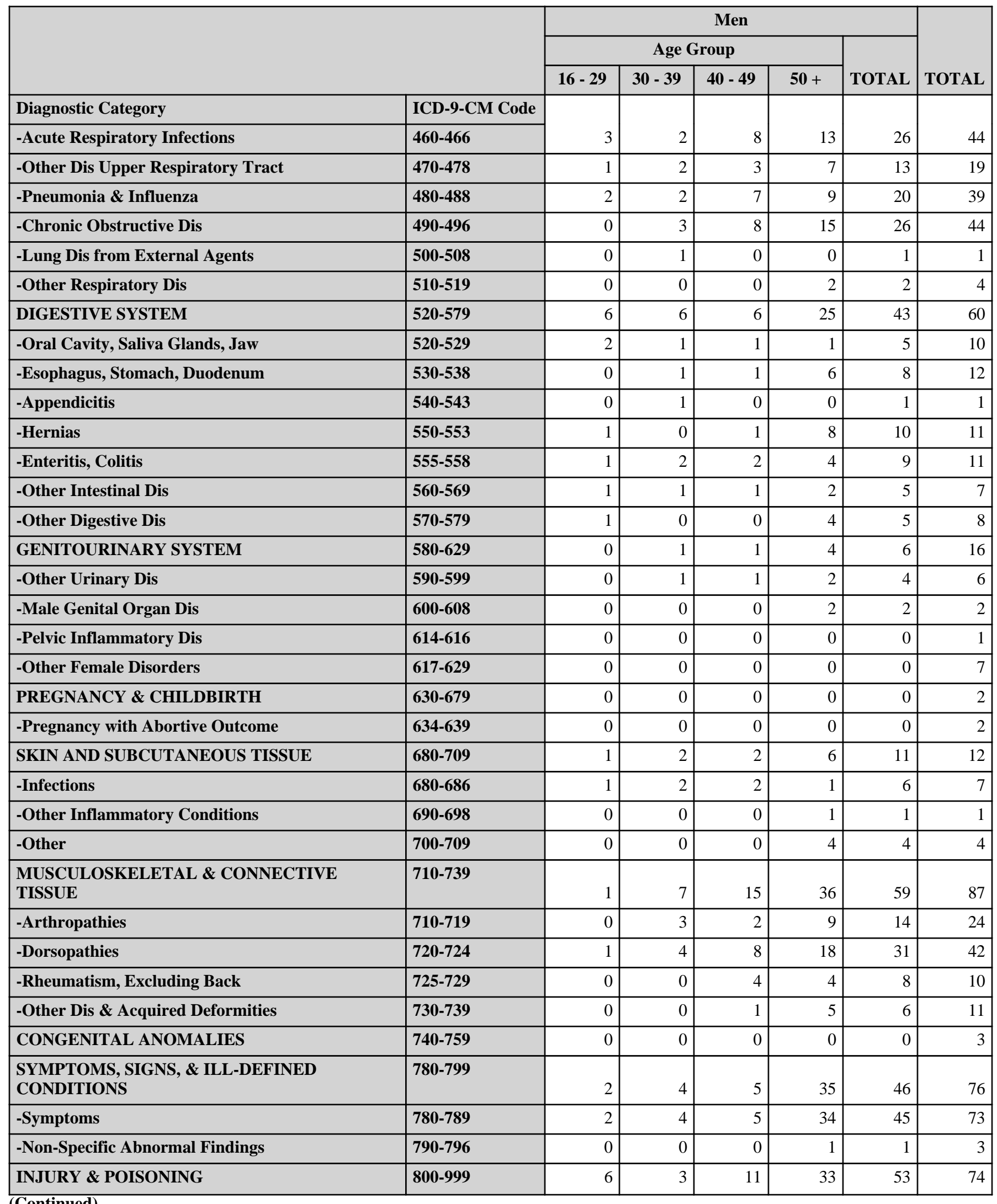

(Continued)

*Only those diagnostic categories and gender/age combinations with at least one occurrence appear in this table. 
Argonne National Laboratory 2009

Absence Data

Appendix G. Number of Diagnoses in Each Diagnostic Category by Gender and Age*

\begin{tabular}{|c|c|c|c|c|c|c|}
\hline & & \multicolumn{5}{|c|}{ Women } \\
\hline & & \multicolumn{4}{|c|}{ Age Group } & \multirow[b]{2}{*}{ TOTAL } \\
\hline & & $16-29$ & $30-39$ & $40-49$ & $50+$ & \\
\hline Diagnostic Category & ICD-9-CM Code & \multirow[b]{2}{*}{0} & \multirow[b]{2}{*}{0} & \multirow[b]{2}{*}{0} & \multirow[b]{2}{*}{0} & \multirow[b]{2}{*}{0} \\
\hline -Fracture - Neck, Trunk & $805-809$ & & & & & \\
\hline -Fracture - Upper Limb & $810-819$ & 0 & 0 & 1 & 0 & 1 \\
\hline -Fracture - Lower Limb & $820-829$ & 0 & 0 & 0 & 3 & 3 \\
\hline -Dislocation & 830-839 & 0 & 0 & 1 & 2 & 3 \\
\hline -Sprains \& Strains - Back & 846-847 & 0 & 0 & 0 & 0 & 0 \\
\hline -Sprains \& Strains - Other & $840-845,848$ & 0 & 0 & 2 & 2 & 4 \\
\hline -Intracranial Injury & $850-854$ & 0 & 0 & 0 & 0 & 0 \\
\hline -Open Wound - Upper Limb & $880-887$ & 0 & 0 & 0 & 0 & 0 \\
\hline -Open Wound - Lower Limb & 890-897 & 0 & 0 & 0 & 0 & 0 \\
\hline -Late Effects of Accident & 905-909 & 0 & 0 & 2 & 0 & 2 \\
\hline -Superficial Injury & $910-919$ & 0 & 0 & 1 & 0 & 1 \\
\hline -Contusion & $920-924$ & 0 & 0 & 0 & 1 & 1 \\
\hline -Burns & 940-949 & 0 & 0 & 0 & 0 & 0 \\
\hline -Complications \& Unspecified Injuries & 958-959 & 0 & 1 & 3 & 1 & 5 \\
\hline -Complications of Surgical/Medical Care & 996-999 & 0 & 0 & 0 & 1 & 1 \\
\hline $\begin{array}{l}\text { HEALTH STATUS/HEALTH SERVICE } \\
\text { CONTACT }\end{array}$ & V01-V89 & 0 & 0 & 0 & 2 & 2 \\
\hline -Specific Procedures/Aftercare & V50-V59 & 0 & 0 & 0 & 0 & 0 \\
\hline -Examination \& Investigation & V70-V82 & 0 & 0 & 0 & 2 & 2 \\
\hline
\end{tabular}

*Only those diagnostic categories and gender/age combinations with at least one occurrence appear in this table. 


\section{Argonne National Laboratory 2009}

\section{Absence Data}

Appendix G. Number of Diagnoses in Each Diagnostic Category by Gender and Age*

\begin{tabular}{|c|c|c|c|c|c|c|c|}
\hline & & \multicolumn{5}{|c|}{ Men } & \multirow[b]{3}{*}{ TOTAL } \\
\hline & & \multicolumn{4}{|c|}{ Age Group } & \multirow[b]{2}{*}{ TOTAL } & \\
\hline & & $16-29$ & $30-39$ & $40-49$ & $50+$ & & \\
\hline -Fracture - Neck, Trunk & $805-809$ & 0 & 0 & 1 & 2 & 3 & 3 \\
\hline -Fracture - Upper Limb & 810-819 & 0 & 0 & 0 & 2 & 2 & 3 \\
\hline -Dislocation & 830-839 & 1 & 0 & 1 & 8 & 10 & 13 \\
\hline -Sprains \& Strains - Back & \begin{tabular}{|l|}
$846-847$ \\
\end{tabular} & 0 & 0 & 1 & 0 & 1 & 1 \\
\hline -Sprains \& Strains - Other & $840-845,848$ & 3 & 1 & 6 & 7 & 17 & 21 \\
\hline -Intracranial Injury & $850-854$ & 0 & 0 & 0 & 2 & 2 & 2 \\
\hline -Superficial Injury & 910-919 & 0 & 0 & 0 & 0 & 0 & 1 \\
\hline -Contusion & $920-924$ & 0 & 0 & 0 & 1 & 1 & 2 \\
\hline -Burns & $940-949$ & 0 & 0 & 0 & 1 & 1 & 1 \\
\hline -Complications \& Unspecified Injuries & 958-959 & 1 & 0 & 0 & 1 & 2 & 7 \\
\hline -Complications of Surgical/Medical Care & 996-999 & 0 & 0 & 0 & 1 & 1 & 2 \\
\hline $\begin{array}{l}\text { HEALTH STATUS/HEALTH SERVICE } \\
\text { CONTACT }\end{array}$ & V01-V89 & 0 & 0 & 0 & 3 & 3 & 5 \\
\hline -Specific Procedures/Aftercare & V50-V59 & 0 & 0 & 0 & 1 & 1 & 1 \\
\hline
\end{tabular}

\begin{tabular}{|c|c|c|c|c|c|c|c|c|c|c|c|}
\hline & \multicolumn{5}{|c|}{ Women } & \multicolumn{5}{|c|}{ Men } & \multirow[b]{3}{*}{ TOTAL } \\
\hline & \multicolumn{4}{|c|}{ Age Group } & \multirow[b]{2}{*}{ TOTAL } & \multicolumn{4}{|c|}{ Age Group } & \multirow[b]{2}{*}{ TOTAL } & \\
\hline & 16 - 29 & 30 - 39 & $40-49$ & $50+$ & & 16 - 29 & 30 - 39 & 40 - 49 & $50+$ & & \\
\hline Diagnostic Category & \multirow[b]{2}{*}{20} & \multirow[b]{2}{*}{11} & \multirow[b]{2}{*}{69} & \multirow[b]{2}{*}{126} & \multirow[b]{2}{*}{226} & \multirow[b]{2}{*}{26} & \multirow[b]{2}{*}{43} & \multirow[b]{2}{*}{88} & \multirow[b]{2}{*}{250} & \multirow[b]{2}{*}{407} & \multirow[b]{2}{*}{633} \\
\hline Total & & & & & & & & & & & \\
\hline
\end{tabular}

*Only those diagnostic categories and gender/age combinations with at least one occurrence appear in this table. 
Argonne National Laboratory 2009

Absence Data

Appendix H. Total Number of Calendar Days Absent in Each Diagnostic Category by Gender and Age*

\begin{tabular}{|c|c|c|c|c|c|c|}
\hline & & \multicolumn{5}{|c|}{ Women } \\
\hline & & \multicolumn{4}{|c|}{ Age Group } & \multirow[b]{2}{*}{ TOTAL } \\
\hline & & $16-29$ & 30 - 39 & $40-49$ & $50+$ & \\
\hline Diagnostic Category & ICD-9-CM Code & \multirow[b]{2}{*}{0} & \multirow[b]{2}{*}{0} & \multirow[b]{2}{*}{16} & \multirow[b]{2}{*}{17} & \multirow[b]{2}{*}{33} \\
\hline INFECTIOUS \& PARASITIC DISEASES (DIS) & 001-139 & & & & & \\
\hline MALIGNANT NEOPLASMS & $\begin{array}{l}140-208 \\
209.0-209.3 \\
230-234\end{array}$ & 0 & 0 & 0 & 210 & 210 \\
\hline BENIGN \& UNCERTAIN NEOPLASMS & \begin{tabular}{|l|}
$209.4-209.6$ \\
$210-229,235-239$
\end{tabular} & 27 & 0 & 41 & 64 & 132 \\
\hline ENDOCRINE/METABOLIC/IMMUNITY & $240-279$ & 27 & 0 & 0 & 30 & 57 \\
\hline BLOOD \& BLOOD-FORMING ORGANS & $280-289$ & 0 & 0 & 0 & 0 & 0 \\
\hline MENTAL DISORDERS & 290-319 & 0 & 0 & 153 & 0 & 153 \\
\hline NERVOUS SYSTEM (NS) \& SENSE ORGANS & 320-389 & 10 & 5 & 26 & 364 & 405 \\
\hline CIRCULATORY SYSTEM & $390-459$ & 26 & 0 & 113 & 57 & 196 \\
\hline RESPIRATORY SYSTEM & $460-519$ & 10 & 13 & 93 & 234 & 350 \\
\hline DIGESTIVE SYSTEM & $520-579$ & 15 & 61 & 31 & 74 & 181 \\
\hline GENITOURINARY SYSTEM & $580-629$ & 15 & 0 & 0 & 289 & 304 \\
\hline PREGNANCY \& CHILDBIRTH & $630-679$ & 5 & 4 & 0 & 0 & 9 \\
\hline SKIN AND SUBCUTANEOUS TISSUE & $680-709$ & 0 & 0 & 0 & 3 & 3 \\
\hline $\begin{array}{l}\text { MUSCULOSKELETAL \& CONNECTIVE } \\
\text { TISSUE }\end{array}$ & $710-739$ & 0 & 3 & 139 & 581 & 723 \\
\hline CONGENITAL ANOMALIES & $740-759$ & 0 & 0 & 88 & 83 & 171 \\
\hline $\begin{array}{l}\text { SYMPTOMS, SIGNS, \& ILL-DEFINED } \\
\text { CONDITIONS }\end{array}$ & 780-799 & 49 & 21 & 32 & 140 & 242 \\
\hline INJURY \& POISONING & $800-999$ & 0 & 9 & 79 & 372 & 460 \\
\hline
\end{tabular}

\footnotetext{
*Absences with >1 ICD-9-CM code in the same diagnostic category were counted only once. Only those diagnostic categories and gender/age combinations with at least one occurrence appear in this table.
} 
Argonne National Laboratory 2009

Absence Data

Appendix H. Total Number of Calendar Days Absent in Each Diagnostic Category by Gender and Age*

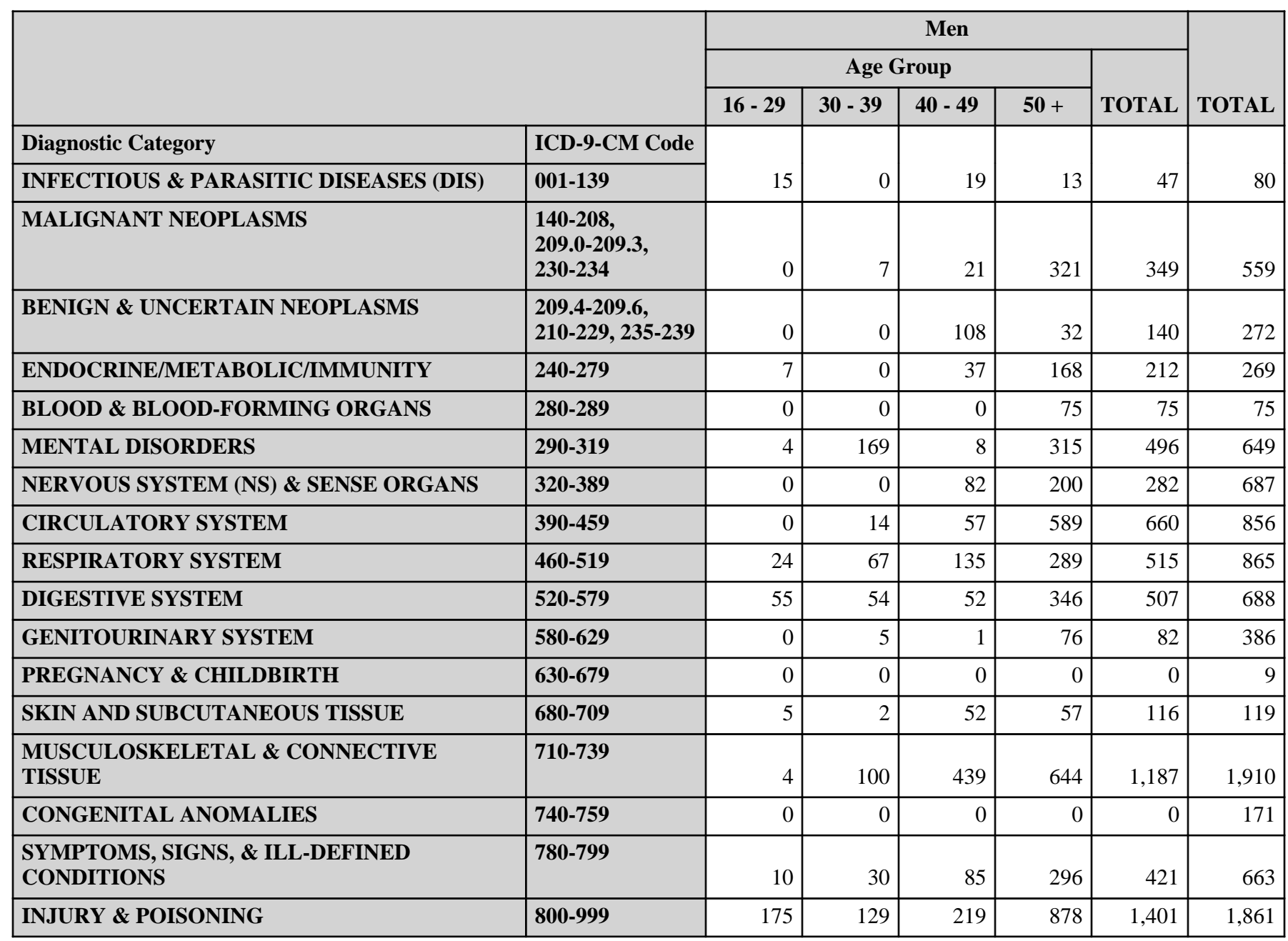

\footnotetext{
*Absences with >1 ICD-9-CM code in the same diagnostic category were counted only once. Only those diagnostic categories and gender/age combinations with at least one occurrence appear in this table.
} 
Argonne National Laboratory 2009

Absence Data

Appendix I. Number of Diagnoses in Each Diagnostic Category by Gender and Job Category*

\begin{tabular}{|c|c|c|c|c|}
\hline & \multicolumn{3}{|c|}{ Women } \\
\hline & & \multicolumn{3}{|c|}{ Job Category } \\
\hline & & Professional & $\begin{array}{c}\text { Administrative } \\
\text { Support }\end{array}$ & Technical Support \\
\hline Diagnostic Category & ICD-9-CM Code & \multirow[b]{2}{*}{3} & \multirow[b]{2}{*}{1} & \multirow[b]{2}{*}{2} \\
\hline INFECTIOUS \& PARASITIC DISEASES (DIS) & 001-139 & & & \\
\hline -Intestinal Infectious Dis & 001-009 & 1 & 0 & 0 \\
\hline -Other Bacterial Dis & 030-041 & 0 & 0 & 1 \\
\hline -Other Viral Dis \& Chlamydiae & 070-079 & 2 & 1 & 1 \\
\hline MALIGNANT NEOPLASMS & $\begin{array}{l}140-208 \\
209.0-209.3 \\
230-234\end{array}$ & 4 & 0 & 0 \\
\hline -Digestive \& Peritoneal & $150-159$ & 1 & 0 & 0 \\
\hline -Breast & 174-175 & 1 & 0 & 0 \\
\hline -Genitourinary & 179-189 & 1 & 0 & 0 \\
\hline -Other \& Unspecified Sites & $195-199,209.7$ & 1 & 0 & 0 \\
\hline BENIGN \& UNCERTAIN NEOPLASMS & \begin{tabular}{|l|}
$209.4-209.6$ \\
$210-229$, \\
$235-239$
\end{tabular} & 1 & 2 & 0 \\
\hline ENDOCRINE/METABOLIC/IMMUNITY & $240-279$ & 1 & 4 & 0 \\
\hline -Other Endocrine Gland Dis & $249-259$ & 0 & 4 & 0 \\
\hline -Other Metabolic \& Immunity Disorders & $270-279$ & 1 & 0 & 0 \\
\hline MENTAL DISORDERS & 290-319 & 0 & 5 & 2 \\
\hline -Non-Psychotic Disorders & $300-302,306-316$ & 0 & 5 & 2 \\
\hline NERVOUS SYSTEM (NS) \& SENSE ORGANS & 320-389 & 3 & 6 & 1 \\
\hline -Other Disorders of Central NS & $340-349$ & 0 & 2 & 1 \\
\hline -Disorders of Peripheral NS & $350-359$ & 1 & 2 & 0 \\
\hline -Disorders of Eye & $360-379$ & 2 & 0 & 0 \\
\hline -Diseases of Ear \& Mastoid & $380-389$ & 0 & 2 & 0 \\
\hline CIRCULATORY SYSTEM & $390-459$ & 2 & 3 & 3 \\
\hline -Hypertensive Dis & $401-405$ & 0 & 1 & 0 \\
\hline -Ischemic Heart Dis & $410-414$ & 0 & 2 & 0 \\
\hline -Other Heart Dis & $420-429$ & 1 & 0 & 0 \\
\hline -Cerebrovascular Dis & $430-438$ & 1 & 0 & 1 \\
\hline -Dis of Veins, Lymphatics, Other & $451-459$ & 0 & 0 & 2 \\
\hline RESPIRATORY SYSTEM & $460-519$ & 23 & 26 & 2 \\
\hline -Acute Respiratory Infections & $460-466$ & 5 & 9 & 0 \\
\hline -Other Dis Upper Respiratory Tract & $470-478$ & 3 & 3 & 0 \\
\hline -Pneumonia \& Influenza & $480-488$ & 8 & 7 & 1 \\
\hline -Chronic Obstructive Dis & $490-496$ & 7 & 7 & 1 \\
\hline -Other Respiratory Dis & $510-519$ & 0 & 0 & 0 \\
\hline DIGESTIVE SYSTEM & $520-579$ & 4 & 8 & 2 \\
\hline -Oral Cavity, Saliva Glands, Jaw & $520-529$ & 3 & 0 & 1 \\
\hline
\end{tabular}

(Continued)

*Only those diagnostic categories and gender/job category combinations with at least one occurrence appear in this table. 
Argonne National Laboratory 2009

Absence Data

Appendix I. Number of Diagnoses in Each Diagnostic Category by Gender and Job Category*

\begin{tabular}{|c|c|c|c|c|c|}
\hline & & \multicolumn{4}{|c|}{ Women } \\
\hline & & \multicolumn{3}{|c|}{ Job Category } & \multirow[b]{2}{*}{ TOTAL } \\
\hline & & Service & Crafts & Line Operators & \\
\hline Diagnostic Category & ICD-9-CM Code & \multirow[b]{2}{*}{1} & \multirow[b]{2}{*}{0} & \multirow[b]{2}{*}{0} & \multirow[b]{2}{*}{7} \\
\hline INFECTIOUS \& PARASITIC DISEASES (DIS) & 001-139 & & & & \\
\hline -Intestinal Infectious Dis & 001-009 & 0 & 0 & 0 & 1 \\
\hline -Other Bacterial Dis & 030-041 & 0 & 0 & 0 & 1 \\
\hline -Other Viral Dis \& Chlamydiae & 070-079 & 1 & 0 & 0 & 5 \\
\hline MALIGNANT NEOPLASMS & $\begin{array}{l}140-208, \\
209.0-209.3 \\
230-234\end{array}$ & 0 & 0 & 0 & 4 \\
\hline -Digestive \& Peritoneal & $150-159$ & 0 & 0 & 0 & 1 \\
\hline -Breast & 174-175 & 0 & 0 & 0 & 1 \\
\hline -Genitourinary & 179-189 & 0 & 0 & 0 & 1 \\
\hline -Other \& Unspecified Sites & 195-199, 209.7 & 0 & 0 & 0 & 1 \\
\hline BENIGN \& UNCERTAIN NEOPLASMS & $\begin{array}{l}209.4-209.6, \\
210-229,235-239\end{array}$ & 0 & 0 & 0 & 3 \\
\hline ENDOCRINE/METABOLIC/IMMUNITY & $240-279$ & 0 & 0 & 0 & 5 \\
\hline -Other Endocrine Gland Dis & 249-259 & 0 & 0 & 0 & 4 \\
\hline -Other Metabolic \& Immunity Disorders & $270-279$ & 0 & 0 & 0 & 1 \\
\hline MENTAL DISORDERS & $290-319$ & 1 & 0 & 0 & 8 \\
\hline -Non-Psychotic Disorders & $300-302,306-316$ & 1 & 0 & 0 & 8 \\
\hline NERVOUS SYSTEM (NS) \& SENSE ORGANS & $320-389$ & 2 & 0 & 0 & 12 \\
\hline -Other Disorders of Central NS & $340-349$ & 0 & 0 & 0 & 3 \\
\hline -Disorders of Peripheral NS & $350-359$ & 2 & 0 & 0 & 5 \\
\hline -Disorders of Eye & $360-379$ & 0 & 0 & 0 & 2 \\
\hline -Diseases of Ear \& Mastoid & $380-389$ & 0 & 0 & 0 & 2 \\
\hline CIRCULATORY SYSTEM & $390-459$ & 2 & 0 & 0 & 10 \\
\hline -Hypertensive Dis & $401-405$ & 1 & 0 & 0 & 2 \\
\hline -Ischemic Heart Dis & $410-414$ & 0 & 0 & 0 & 2 \\
\hline -Other Heart Dis & $420-429$ & 1 & 0 & 0 & 2 \\
\hline -Cerebrovascular Dis & $430-438$ & 0 & 0 & 0 & 2 \\
\hline -Dis of Veins, Lymphatics, Other & $451-459$ & 0 & 0 & 0 & 2 \\
\hline RESPIRATORY SYSTEM & $460-519$ & 8 & 2 & 2 & 63 \\
\hline -Acute Respiratory Infections & $460-466$ & 4 & 0 & 0 & 18 \\
\hline -Other Dis Upper Respiratory Tract & $470-478$ & 0 & 0 & 0 & 6 \\
\hline -Pneumonia \& Influenza & $480-488$ & 2 & 0 & 1 & 19 \\
\hline -Chronic Obstructive Dis & $490-496$ & 1 & 1 & 1 & 18 \\
\hline -Other Respiratory Dis & $510-519$ & 1 & 1 & 0 & 2 \\
\hline DIGESTIVE SYSTEM & $520-579$ & 3 & 0 & 0 & 17 \\
\hline -Oral Cavity, Saliva Glands, Jaw & $520-529$ & 1 & 0 & \begin{tabular}{l|l}
0 \\
\end{tabular} & 5 \\
\hline
\end{tabular}

(Continued)

*Only those diagnostic categories and gender/job category combinations with at least one occurrence appear in this table. 
Argonne National Laboratory 2009

Absence Data

Appendix I. Number of Diagnoses in Each Diagnostic Category by Gender and Job Category*

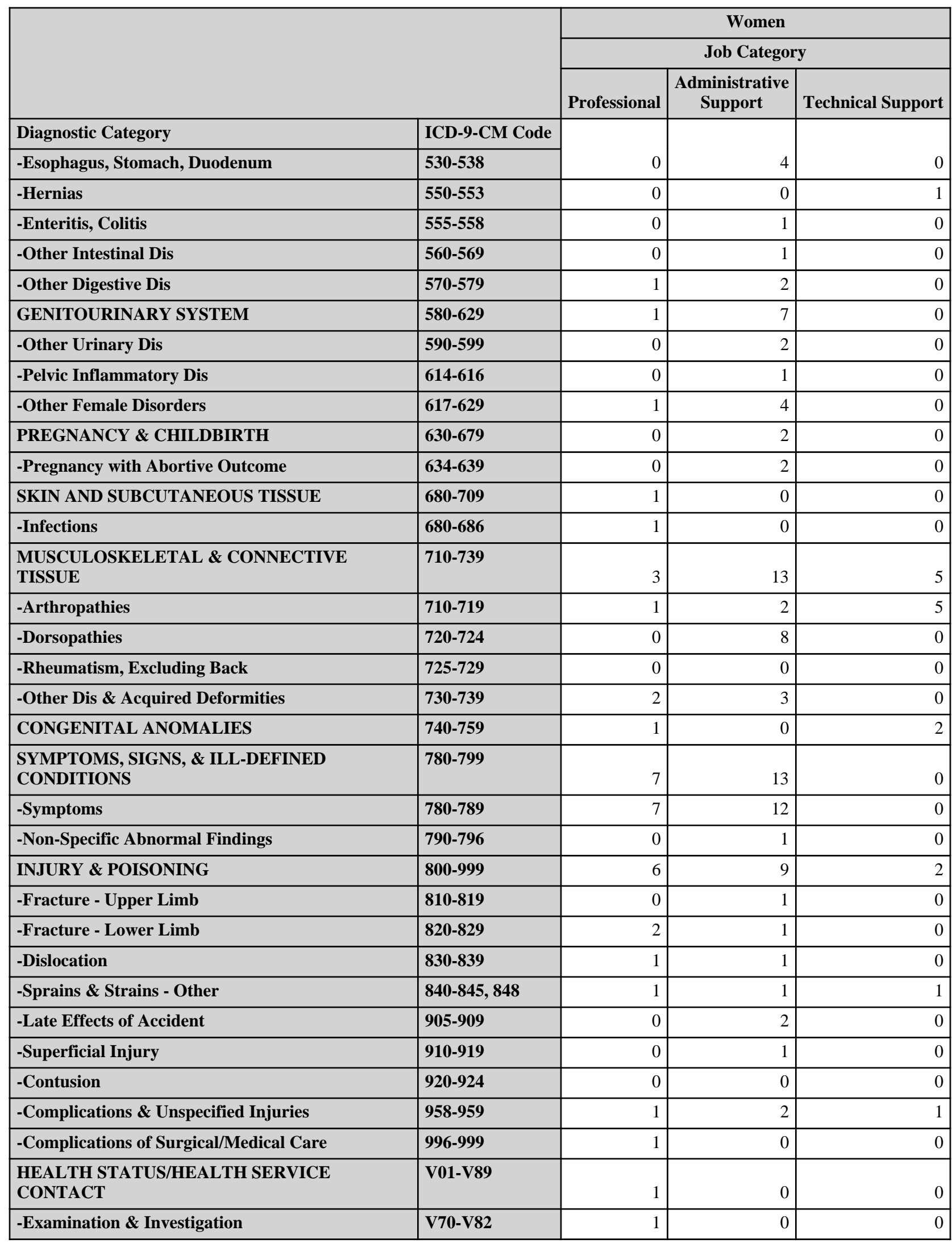

*Only those diagnostic categories and gender/job category combinations with at least one occurrence appear in this table. 
Argonne National Laboratory 2009

Absence Data

Appendix I. Number of Diagnoses in Each Diagnostic Category by Gender and Job Category*

\begin{tabular}{|c|c|c|c|c|c|}
\hline & & \multicolumn{4}{|c|}{ Women } \\
\hline & & \multicolumn{3}{|c|}{ Job Category } & \multirow[b]{2}{*}{ TOTAL } \\
\hline & & Service & Crafts & Line Operators & \\
\hline Diagnostic Category & ICD-9-CM Code & \multirow[b]{2}{*}{0} & \multirow[b]{2}{*}{0} & \multirow[b]{2}{*}{0} & \multirow[b]{2}{*}{4} \\
\hline -Esophagus, Stomach, Duodenum & $530-538$ & & & & \\
\hline -Hernias & $550-553$ & 0 & 0 & 0 & 1 \\
\hline -Enteritis, Colitis & $555-558$ & 1 & 0 & 0 & 2 \\
\hline -Other Intestinal Dis & $560-569$ & 1 & 0 & 0 & 2 \\
\hline -Other Digestive Dis & $570-579$ & 0 & 0 & 0 & 3 \\
\hline GENITOURINARY SYSTEM & $580-629$ & 0 & 2 & 0 & 10 \\
\hline -Other Urinary Dis & $590-599$ & 0 & 0 & 0 & 2 \\
\hline -Pelvic Inflammatory Dis & 614-616 & 0 & 0 & 0 & 1 \\
\hline -Other Female Disorders & $617-629$ & 0 & 2 & 0 & 7 \\
\hline PREGNANCY \& CHILDBIRTH & $630-679$ & 0 & 0 & 0 & 2 \\
\hline -Pregnancy with Abortive Outcome & $634-639$ & 0 & 0 & 0 & 2 \\
\hline SKIN AND SUBCUTANEOUS TISSUE & $680-709$ & 0 & 0 & 0 & 1 \\
\hline -Infections & $680-686$ & 0 & 0 & 0 & 1 \\
\hline $\begin{array}{l}\text { MUSCULOSKELETAL \& CONNECTIVE } \\
\text { TISSUE }\end{array}$ & $710-739$ & 6 & 1 & 0 & 28 \\
\hline -Arthropathies & $710-719$ & 2 & 0 & 0 & 10 \\
\hline -Dorsopathies & $720-724$ & 2 & 1 & 0 & 11 \\
\hline -Rheumatism, Excluding Back & 725-729 & 2 & 0 & 0 & 2 \\
\hline -Other Dis \& Acquired Deformities & 730-739 & 0 & 0 & 0 & 5 \\
\hline CONGENITAL ANOMALIES & $740-759$ & 0 & 0 & 0 & 3 \\
\hline $\begin{array}{l}\text { SYMPTOMS, SIGNS, \& ILL-DEFINED } \\
\text { CONDITIONS }\end{array}$ & $780-799$ & 5 & 0 & 5 & 30 \\
\hline -Symptoms & \begin{tabular}{|l|}
$780-789$ \\
\end{tabular} & 4 & 0 & 5 & 28 \\
\hline -Non-Specific Abnormal Findings & $790-796$ & 1 & 0 & 0 & 2 \\
\hline INJURY \& POISONING & $800-999$ & 4 & 0 & 0 & 21 \\
\hline -Fracture - Upper Limb & 810-819 & 0 & 0 & 0 & 1 \\
\hline -Fracture - Lower Limb & $820-829$ & 0 & 0 & 0 & 3 \\
\hline -Dislocation & $830-839$ & 1 & 0 & 0 & 3 \\
\hline -Sprains \& Strains - Other & $840-845,848$ & 1 & 0 & 0 & 4 \\
\hline -Late Effects of Accident & $905-909$ & 0 & 0 & 0 & 2 \\
\hline -Superficial Injury & 910-919 & 0 & 0 & 0 & 1 \\
\hline -Contusion & 920-924 & 1 & 0 & 0 & 1 \\
\hline -Complications \& Unspecified Injuries & 958-959 & 1 & 0 & 0 & 5 \\
\hline -Complications of Surgical/Medical Care & 996-999 & 0 & 0 & 0 & 1 \\
\hline $\begin{array}{l}\text { HEALTH STATUS/HEALTH SERVICE } \\
\text { CONTACT }\end{array}$ & V01-V89 & 1 & 0 & 0 & 2 \\
\hline -Examination \& Investigation & V70-V82 & 1 & 0 & 0 & 2 \\
\hline
\end{tabular}

*Only those diagnostic categories and gender/job category combinations with at least one occurrence appear in this table. 
Argonne National Laboratory 2009

Absence Data

Appendix I. Number of Diagnoses in Each Diagnostic Category by Gender and Job Category*

\begin{tabular}{|c|c|c|c|c|c|c|c|}
\hline & \multicolumn{7}{|c|}{ Women } \\
\hline & \multicolumn{6}{|c|}{ Job Category } & \multirow[b]{2}{*}{ TOTAL } \\
\hline & Professional & $\begin{array}{c}\text { Administrative } \\
\text { Support }\end{array}$ & Technical Support & Service & Crafts & Line Operators & \\
\hline Diagnostic Category & \multirow[b]{2}{*}{61} & \multirow[b]{2}{*}{99} & \multirow[b]{2}{*}{21} & \multirow[b]{2}{*}{33} & \multirow[b]{2}{*}{5} & \multirow[b]{2}{*}{7} & \multirow[b]{2}{*}{226} \\
\hline Total & & & & & & & \\
\hline
\end{tabular}

*Only those diagnostic categories and gender/job category combinations with at least one occurrence appear in this table. 
Argonne National Laboratory 2009

Absence Data

Appendix I. Number of Diagnoses in Each Diagnostic Category by Gender and Job Category*

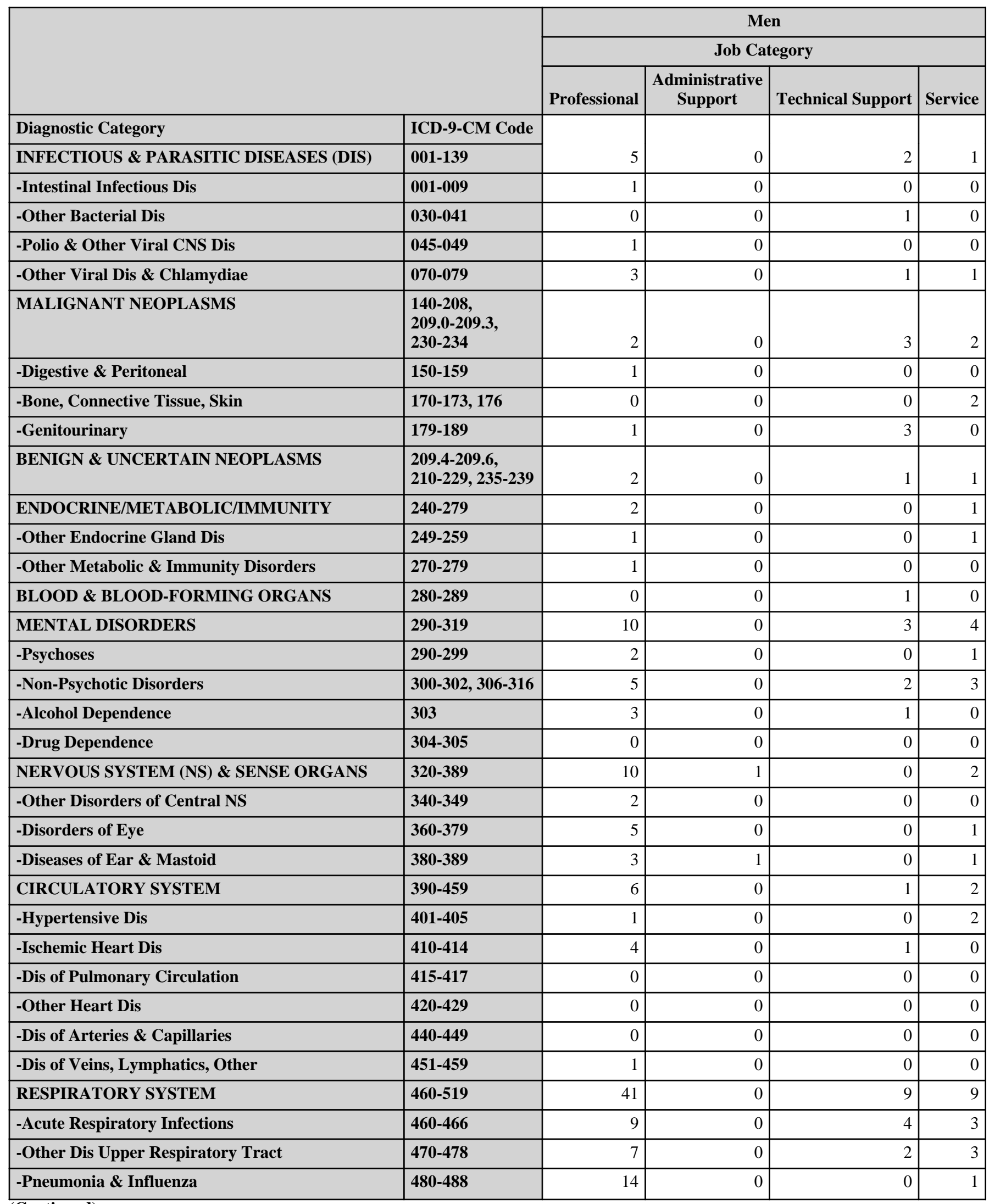

(Continued)

*Only those diagnostic categories and gender/job category combinations with at least one occurrence appear in this table. 
Argonne National Laboratory 2009

Absence Data

Appendix I. Number of Diagnoses in Each Diagnostic Category by Gender and Job Category*

\begin{tabular}{|c|c|c|c|c|c|}
\hline & & \multicolumn{4}{|c|}{ Men } \\
\hline & & \multicolumn{3}{|c|}{ Job Category } & \multirow[b]{2}{*}{ TOTAL } \\
\hline & & Security and Fire & Crafts & Line Operators & \\
\hline Diagnostic Category & ICD-9-CM Code & \multirow[b]{2}{*}{0} & \multirow[b]{2}{*}{1} & \multirow[b]{2}{*}{1} & \multirow[b]{2}{*}{10} \\
\hline INFECTIOUS \& PARASITIC DISEASES (DIS) & 001-139 & & & & \\
\hline -Intestinal Infectious Dis & 001-009 & 0 & 0 & 0 & 1 \\
\hline -Other Bacterial Dis & 030-041 & 0 & 0 & 0 & 1 \\
\hline -Polio \& Other Viral CNS Dis & 045-049 & 0 & 0 & 0 & 1 \\
\hline -Other Viral Dis \& Chlamydiae & 070-079 & 0 & 1 & 1 & 7 \\
\hline MALIGNANT NEOPLASMS & \begin{tabular}{|l|}
$140-208$ \\
$209.0-209.3$ \\
$230-234$
\end{tabular} & 0 & 4 & 0 & 11 \\
\hline -Digestive \& Peritoneal & $150-159$ & 0 & 0 & 0 & 1 \\
\hline -Bone, Connective Tissue, Skin & 170-173, 176 & 0 & 0 & 0 & 2 \\
\hline -Genitourinary & 179-189 & 0 & 4 & 0 & 8 \\
\hline BENIGN \& UNCERTAIN NEOPLASMS & \begin{tabular}{|l|}
$209.4-209.6$ \\
$210-229,235-239$
\end{tabular} & 0 & 0 & 0 & 4 \\
\hline ENDOCRINE/METABOLIC/IMMUNITY & $240-279$ & 1 & 3 & 0 & 7 \\
\hline -Other Endocrine Gland Dis & $249-259$ & 0 & 1 & 0 & 3 \\
\hline -Other Metabolic \& Immunity Disorders & 270-279 & 1 & 2 & 0 & 4 \\
\hline BLOOD \& BLOOD-FORMING ORGANS & $280-289$ & 0 & 1 & 0 & 2 \\
\hline MENTAL DISORDERS & 290-319 & 0 & 11 & 0 & 28 \\
\hline -Psychoses & $290-299$ & 0 & 0 & 0 & 3 \\
\hline -Non-Psychotic Disorders & $300-302,306-316$ & 0 & 8 & 0 & 18 \\
\hline -Alcohol Dependence & 303 & 0 & 0 & 0 & 4 \\
\hline -Drug Dependence & 304-305 & 0 & 3 & 0 & 3 \\
\hline NERVOUS SYSTEM (NS) \& SENSE ORGANS & $320-389$ & 0 & 3 & 1 & 17 \\
\hline -Other Disorders of Central NS & 340-349 & 0 & 0 & 0 & 2 \\
\hline -Disorders of Eye & $360-379$ & 0 & 1 & 0 & $\overline{7}$ \\
\hline -Diseases of Ear \& Mastoid & 380-389 & 0 & 2 & 1 & 8 \\
\hline CIRCULATORY SYSTEM & $390-459$ & 0 & 9 & 1 & 19 \\
\hline -Hypertensive Dis & 401-405 & 0 & 4 & 0 & 7 \\
\hline -Ischemic Heart Dis & $410-414$ & 0 & 2 & 0 & $\overline{7}$ \\
\hline -Dis of Pulmonary Circulation & $415-417$ & 0 & 1 & 0 & 1 \\
\hline -Other Heart Dis & $420-429$ & 0 & 1 & 1 & 2 \\
\hline -Dis of Arteries \& Capillaries & $440-449$ & 0 & 1 & 0 & 1 \\
\hline -Dis of Veins, Lymphatics, Other & 451-459 & 0 & 0 & 0 & 1 \\
\hline RESPIRATORY SYSTEM & $460-519$ & 1 & 26 & 2 & 88 \\
\hline -Acute Respiratory Infections & $460-466$ & 0 & 8 & 2 & 26 \\
\hline -Other Dis Upper Respiratory Tract & $470-478$ & 0 & 1 & 0 & 13 \\
\hline -Pneumonia \& Influenza & $480-488$ & 0 & 5 & 0 & 20 \\
\hline
\end{tabular}

(Continued)

*Only those diagnostic categories and gender/job category combinations with at least one occurrence appear in this table. 


\section{Argonne National Laboratory 2009}

\section{Absence Data}

Appendix I. Number of Diagnoses in Each Diagnostic Category by Gender and Job Category*

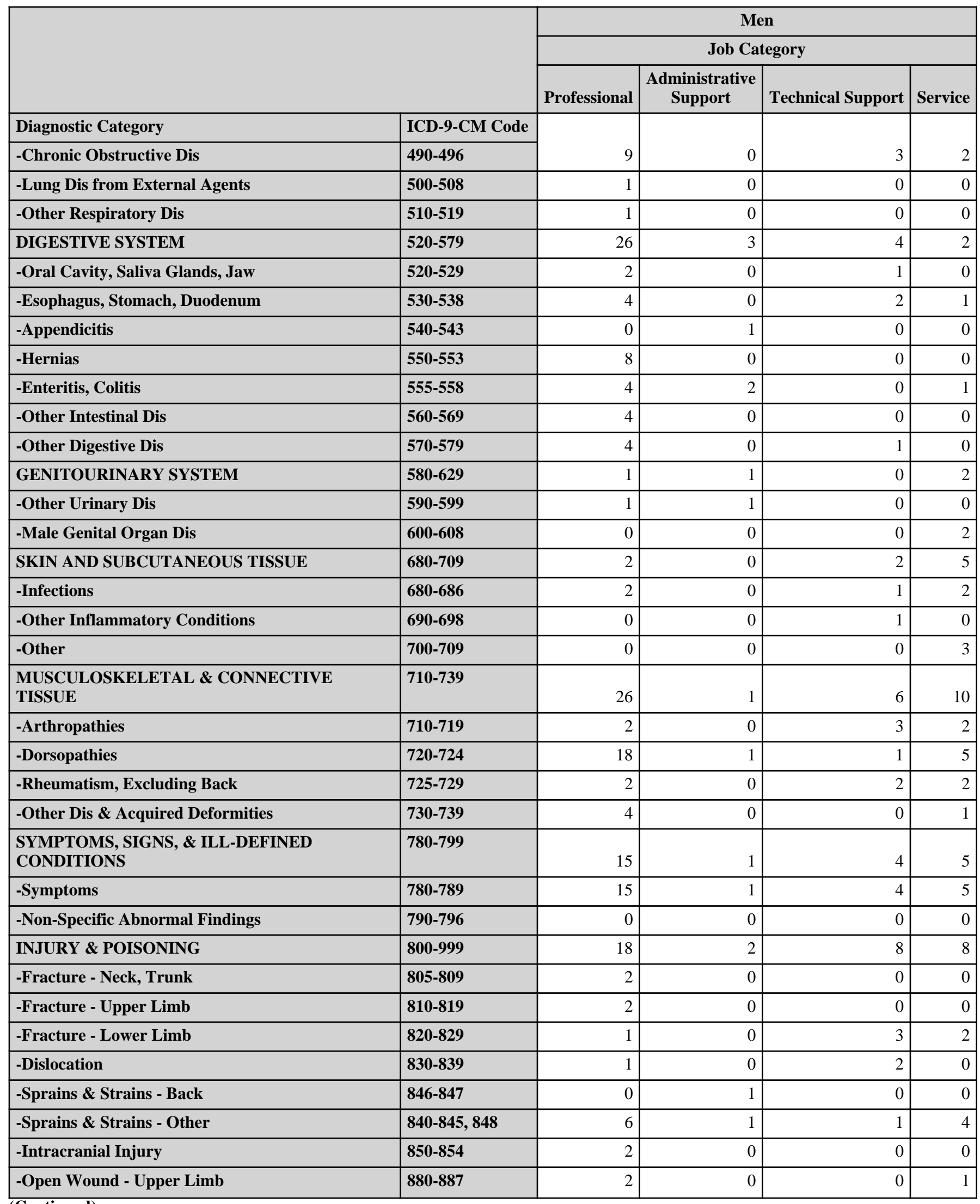

(Continued)

*Only those diagnostic categories and gender/job category combinations with at least one occurrence appear in this table. 
Argonne National Laboratory 2009

Absence Data

Appendix I. Number of Diagnoses in Each Diagnostic Category by Gender and Job Category*

\begin{tabular}{|c|c|c|c|c|c|}
\hline & & \multicolumn{4}{|c|}{ Men } \\
\hline & & \multicolumn{3}{|c|}{ Job Category } & \multirow[b]{2}{*}{ TOTAL } \\
\hline & & Security and Fire & Crafts & Line Operators & \\
\hline Diagnostic Category & ICD-9-CM Code & \multirow[b]{2}{*}{1} & \multirow[b]{2}{*}{11} & \multirow[b]{2}{*}{0} & \multirow[b]{2}{*}{26} \\
\hline -Chronic Obstructive Dis & $490-496$ & & & & \\
\hline -Lung Dis from External Agents & $500-508$ & 0 & 0 & 0 & 1 \\
\hline -Other Respiratory Dis & $510-519$ & 0 & 1 & 0 & 2 \\
\hline DIGESTIVE SYSTEM & $520-579$ & 0 & 8 & 0 & 43 \\
\hline -Oral Cavity, Saliva Glands, Jaw & $520-529$ & 0 & 2 & 0 & 5 \\
\hline -Esophagus, Stomach, Duodenum & $530-538$ & 0 & 1 & 0 & 8 \\
\hline -Appendicitis & $540-543$ & 0 & 0 & 0 & 1 \\
\hline -Hernias & $550-553$ & 0 & 2 & 0 & 10 \\
\hline -Enteritis, Colitis & $555-558$ & 0 & 2 & 0 & 9 \\
\hline -Other Intestinal Dis & $560-569$ & 0 & 1 & 0 & 5 \\
\hline -Other Digestive Dis & $570-579$ & 0 & 0 & 0 & 5 \\
\hline GENITOURINARY SYSTEM & $580-629$ & 0 & 2 & 0 & 6 \\
\hline -Other Urinary Dis & $590-599$ & 0 & 2 & 0 & 4 \\
\hline -Male Genital Organ Dis & $600-608$ & 0 & 0 & 0 & 2 \\
\hline SKIN AND SUBCUTANEOUS TISSUE & $680-709$ & 0 & 1 & 1 & 11 \\
\hline -Infections & $680-686$ & 0 & 0 & 1 & 6 \\
\hline -Other Inflammatory Conditions & $690-698$ & 0 & 0 & 0 & 1 \\
\hline -Other & 700-709 & 0 & 1 & 0 & 4 \\
\hline $\begin{array}{l}\text { MUSCULOSKELETAL \& CONNECTIVE } \\
\text { TISSUE }\end{array}$ & $710-739$ & 2 & 13 & 1 & 59 \\
\hline -Arthropathies & $710-719$ & 0 & 6 & 1 & 14 \\
\hline -Dorsopathies & $720-724$ & 1 & 5 & 0 & 31 \\
\hline -Rheumatism, Excluding Back & 725-729 & 0 & 2 & 0 & 8 \\
\hline -Other Dis \& Acquired Deformities & $730-739$ & 1 & 0 & 0 & 6 \\
\hline $\begin{array}{l}\text { SYMPTOMS, SIGNS, \& ILL-DEFINED } \\
\text { CONDITIONS }\end{array}$ & 780-799 & 2 & 18 & 1 & 46 \\
\hline -Symptoms & 780-789 & 2 & 18 & 0 & 45 \\
\hline -Non-Specific Abnormal Findings & $790-796$ & 0 & 0 & 1 & 1 \\
\hline INJURY \& POISONING & 800-999 & 2 & 14 & 1 & 53 \\
\hline -Fracture - Neck, Trunk & $805-809$ & 0 & 1 & 0 & 3 \\
\hline -Fracture - Upper Limb & 810-819 & 0 & 0 & 0 & 2 \\
\hline -Fracture - Lower Limb & $820-829$ & 0 & 1 & 0 & 7 \\
\hline -Dislocation & 830-839 & 2 & 5 & 0 & 10 \\
\hline -Sprains \& Strains - Back & 846-847 & 0 & 0 & 0 & 1 \\
\hline -Sprains \& Strains - Other & $840-845,848$ & 0 & 5 & 0 & 17 \\
\hline -Intracranial Injury & $850-854$ & 0 & 0 & 0 & 2 \\
\hline -Open Wound - Upper Limb & $880-887$ & 0 & 0 & 1 & 4 \\
\hline
\end{tabular}

(Continued)

*Only those diagnostic categories and gender/job category combinations with at least one occurrence appear in this table. 
Argonne National Laboratory 2009

Absence Data

Appendix I. Number of Diagnoses in Each Diagnostic Category by Gender and Job Category*

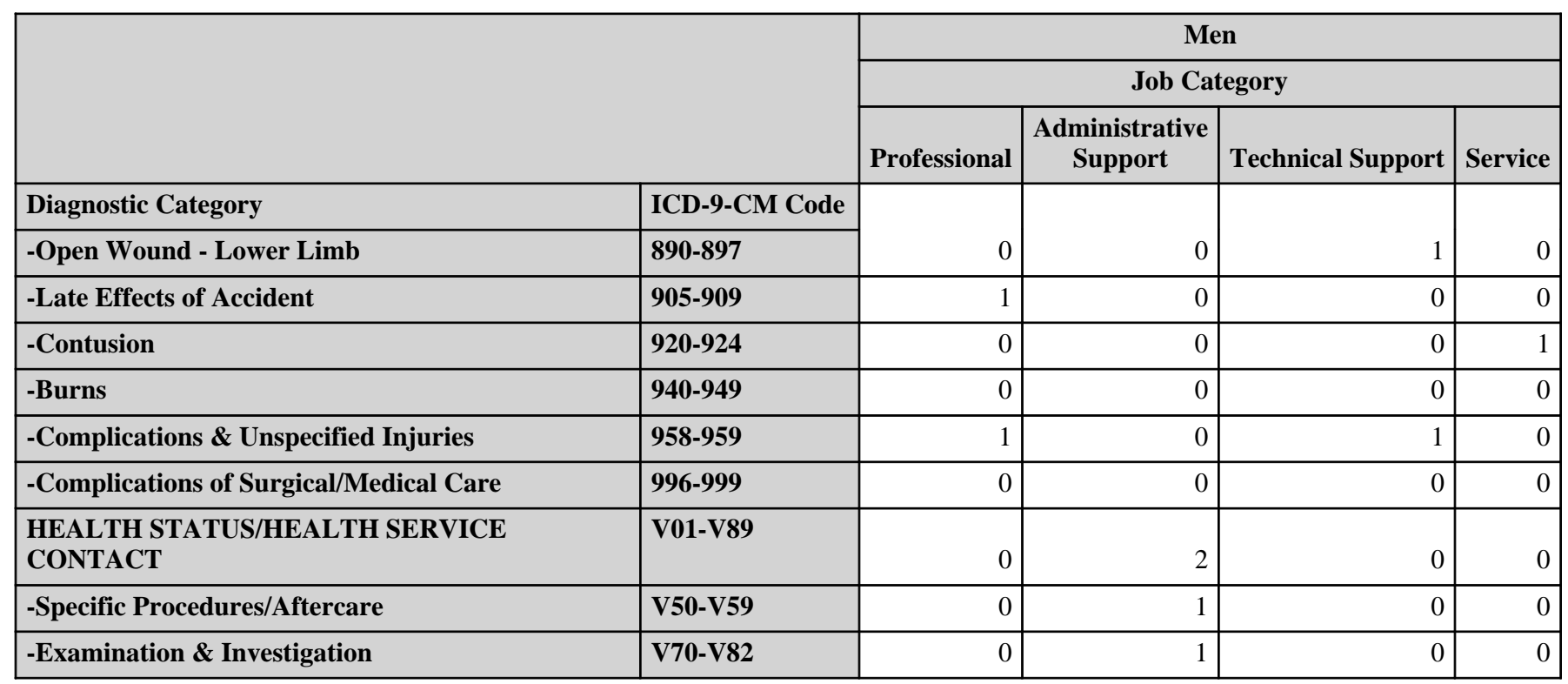

*Only those diagnostic categories and gender/job category combinations with at least one occurrence appear in this table. 
Argonne National Laboratory 2009

Absence Data

Appendix I. Number of Diagnoses in Each Diagnostic Category by Gender and Job Category*

\begin{tabular}{|c|c|c|c|c|c|}
\hline & & \multicolumn{4}{|c|}{ Men } \\
\hline & & \multicolumn{3}{|c|}{ Job Category } & \multirow[b]{2}{*}{ TOTAL } \\
\hline & & Security and Fire & Crafts & Line Operators & \\
\hline Diagnostic Category & ICD-9-CM Code & \multirow[b]{2}{*}{0} & \multirow[b]{2}{*}{0} & \multirow[b]{2}{*}{0} & \multirow[b]{2}{*}{1} \\
\hline -Open Wound - Lower Limb & 890-897 & & & & \\
\hline -Late Effects of Accident & 905-909 & 0 & 0 & 0 & 1 \\
\hline -Contusion & 920-924 & 0 & 0 & 0 & 1 \\
\hline -Burns & 940-949 & 0 & 1 & 0 & 1 \\
\hline -Complications \& Unspecified Injuries & 958-959 & 0 & 0 & 0 & 2 \\
\hline -Complications of Surgical/Medical Care & 996-999 & 0 & 1 & 0 & 1 \\
\hline $\begin{array}{l}\text { HEALTH STATUS/HEALTH SERVICE } \\
\text { CONTACT }\end{array}$ & V01-V89 & 0 & 1 & 0 & 3 \\
\hline -Specific Procedures/Aftercare & V50-V59 & 0 & 0 & 0 & 1 \\
\hline -Examination \& Investigation & V70-V82 & 0 & 1 & 0 & 2 \\
\hline
\end{tabular}

\begin{tabular}{|c|c|c|c|c|c|c|c|c|}
\hline & \multicolumn{8}{|c|}{ Men } \\
\hline & \multicolumn{7}{|c|}{ Job Category } & \multirow[b]{2}{*}{ TOTAL } \\
\hline & Professional & $\begin{array}{c}\text { Administrative } \\
\text { Support }\end{array}$ & $\begin{array}{l}\text { Technical } \\
\text { Support }\end{array}$ & Service & $\begin{array}{l}\text { Security and } \\
\text { Fire }\end{array}$ & Crafts & $\begin{array}{c}\text { Line } \\
\text { Operators }\end{array}$ & \\
\hline Diagnostic Category & \multirow[b]{2}{*}{166} & \multirow[b]{2}{*}{11} & \multirow[b]{2}{*}{44} & \multirow[b]{2}{*}{54} & \multirow[b]{2}{*}{8} & \multirow[b]{2}{*}{115} & \multirow[b]{2}{*}{9} & \multirow[b]{2}{*}{407} \\
\hline Total & & & & & & & & \\
\hline
\end{tabular}

*Only those diagnostic categories and gender/job category combinations with at least one occurrence appear in this table. 
Argonne National Laboratory 2009

Absence Data

Appendix J. Total Number of Calendar Days Absent in Each Diagnostic Category by Gender and Job Category*

\begin{tabular}{|c|c|c|c|c|}
\hline & & \multicolumn{3}{|c|}{ Women } \\
\hline & & \multicolumn{3}{|c|}{ Job Category } \\
\hline & & Professional & $\begin{array}{l}\text { Administrative } \\
\text { Support }\end{array}$ & Technical Support \\
\hline Diagnostic Category & ICD-9-CM Code & \multirow[b]{2}{*}{16} & \multirow[b]{2}{*}{6} & \multirow[b]{2}{*}{4} \\
\hline INFECTIOUS \& PARASITIC DISEASES (DIS) & 001-139 & & & \\
\hline MALIGNANT NEOPLASMS & $\begin{array}{l}140-208 \\
209.0-209.3 \\
230-234\end{array}$ & 210 & 0 & 0 \\
\hline BENIGN \& UNCERTAIN NEOPLASMS & $\begin{array}{l}209.4-209.6 \\
210-229,235-239\end{array}$ & 41 & 91 & 0 \\
\hline ENDOCRINE/METABOLIC/IMMUNITY & $240-279$ & 3 & 54 & 0 \\
\hline MENTAL DISORDERS & $290-319$ & 0 & 76 & 70 \\
\hline NERVOUS SYSTEM (NS) \& SENSE ORGANS & 320-389 & 36 & 38 & 3 \\
\hline CIRCULATORY SYSTEM & $390-459$ & 39 & 13 & 118 \\
\hline RESPIRATORY SYSTEM & $460-519$ & 127 & 133 & 7 \\
\hline DIGESTIVE SYSTEM & $520-579$ & 28 & 73 & 44 \\
\hline GENITOURINARY SYSTEM & $580-629$ & 147 & 91 & 0 \\
\hline PREGNANCY \& CHILDBIRTH & $630-679$ & 0 & 9 & 0 \\
\hline SKIN AND SUBCUTANEOUS TISSUE & $680-709$ & 3 & 0 & 0 \\
\hline $\begin{array}{l}\text { MUSCULOSKELETAL \& CONNECTIVE } \\
\text { TISSUE }\end{array}$ & $710-739$ & 114 & 236 & 293 \\
\hline CONGENITAL ANOMALIES & $740-759$ & 83 & 0 & 88 \\
\hline $\begin{array}{l}\text { SYMPTOMS, SIGNS, \& ILL-DEFINED } \\
\text { CONDITIONS }\end{array}$ & 780-799 & 43 & 100 & 0 \\
\hline INJURY \& POISONING & $800-999$ & 118 & 129 & 8 \\
\hline
\end{tabular}

\footnotetext{
*Absences with >1 ICD-9-CM code in the same diagnostic category were counted only once. Only those diagnostic categories and gender/job category combinations with at least one occurrence appear in this table.
} 
Argonne National Laboratory 2009

Absence Data

Appendix J. Total Number of Calendar Days Absent in Each Diagnostic Category by Gender and Job Category*

\begin{tabular}{|c|c|c|c|c|c|}
\hline & & \multicolumn{4}{|c|}{ Women } \\
\hline & & \multicolumn{3}{|c|}{ Job Category } & \multirow[b]{2}{*}{ TOTAL } \\
\hline & & Service & Crafts & Line Operators & \\
\hline Diagnostic Category & ICD-9-CM Code & \multirow[b]{2}{*}{7} & \multirow[b]{2}{*}{0} & \multirow[b]{2}{*}{0} & \multirow[b]{2}{*}{33} \\
\hline INFECTIOUS \& PARASITIC DISEASES (DIS) & 001-139 & & & & \\
\hline MALIGNANT NEOPLASMS & $\begin{array}{l}140-208 \\
209.0-209.3 \\
230-234\end{array}$ & 0 & 0 & 0 & 210 \\
\hline BENIGN \& UNCERTAIN NEOPLASMS & $\begin{array}{l}209.4-209.6 \\
210-229,235-239\end{array}$ & 0 & 0 & 0 & 132 \\
\hline ENDOCRINE/METABOLIC/IMMUNITY & $240-279$ & 0 & 0 & 0 & 57 \\
\hline MENTAL DISORDERS & $290-319$ & 7 & 0 & 0 & 153 \\
\hline NERVOUS SYSTEM (NS) \& SENSE ORGANS & 320-389 & 328 & 0 & 0 & 405 \\
\hline CIRCULATORY SYSTEM & $390-459$ & 26 & 0 & 0 & 196 \\
\hline RESPIRATORY SYSTEM & $460-519$ & 57 & 15 & 11 & 350 \\
\hline DIGESTIVE SYSTEM & $520-579$ & 36 & 0 & 0 & 181 \\
\hline GENITOURINARY SYSTEM & $580-629$ & 0 & 66 & 0 & 304 \\
\hline PREGNANCY \& CHILDBIRTH & $630-679$ & 0 & 0 & 0 & 9 \\
\hline SKIN AND SUBCUTANEOUS TISSUE & $680-709$ & 0 & 0 & 0 & 3 \\
\hline $\begin{array}{l}\text { MUSCULOSKELETAL \& CONNECTIVE } \\
\text { TISSUE }\end{array}$ & $710-739$ & 31 & 49 & 0 & 723 \\
\hline CONGENITAL ANOMALIES & $740-759$ & 0 & 0 & 0 & 171 \\
\hline $\begin{array}{l}\text { SYMPTOMS, SIGNS, \& ILL-DEFINED } \\
\text { CONDITIONS }\end{array}$ & 780-799 & 18 & 0 & 81 & 242 \\
\hline INJURY \& POISONING & $800-999$ & 205 & 0 & 0 & 460 \\
\hline
\end{tabular}

\footnotetext{
*Absences with >1 ICD-9-CM code in the same diagnostic category were counted only once. Only those diagnostic categories and gender/job category combinations with at least one occurrence appear in this table.
} 
Argonne National Laboratory 2009

Absence Data

Appendix J. Total Number of Calendar Days Absent in Each Diagnostic Category by Gender and Job Category*

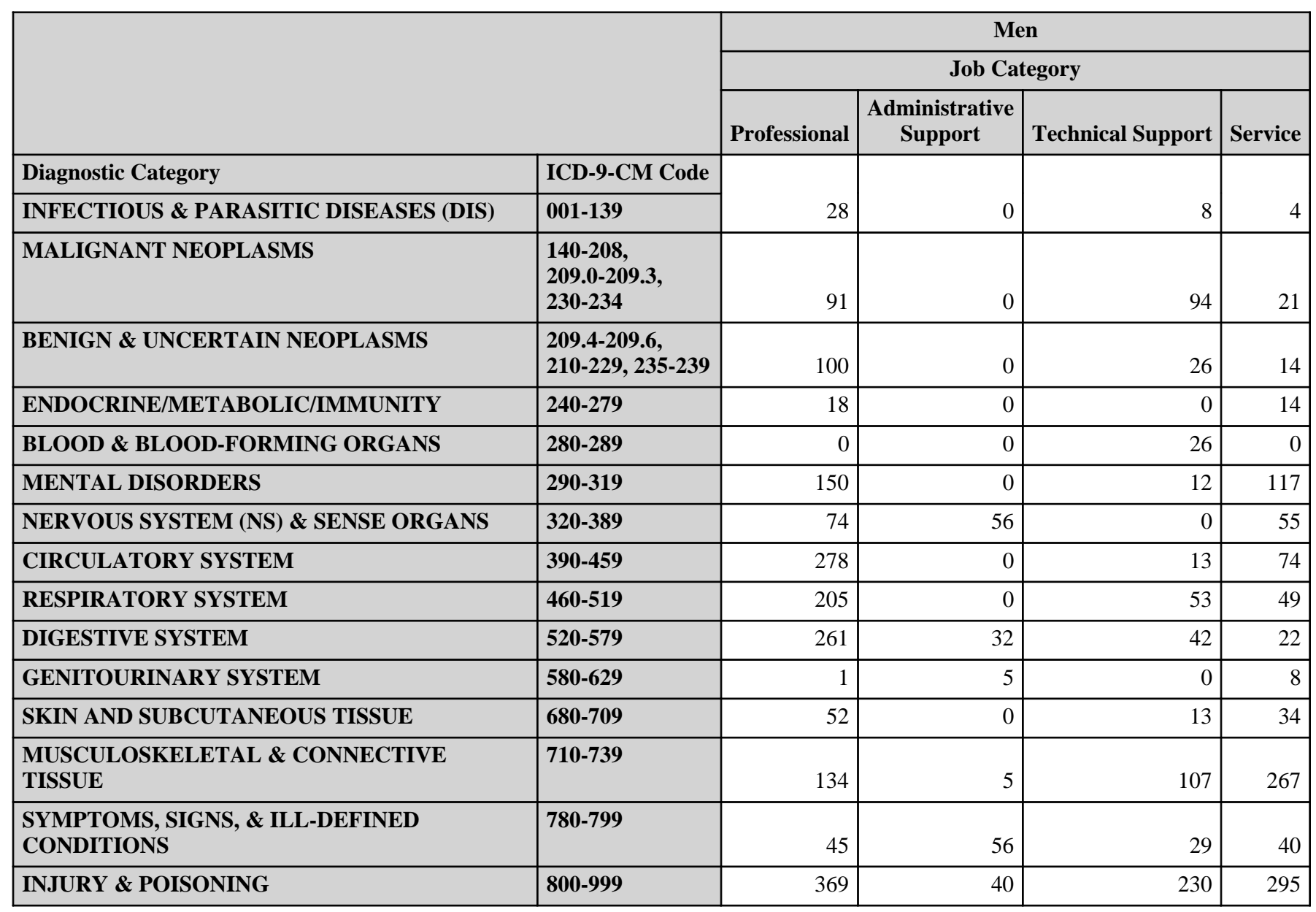

\footnotetext{
*Absences with >1 ICD-9-CM code in the same diagnostic category were counted only once. Only those diagnostic categories and gender/job category combinations with at least one occurrence appear in this table.
} 
Argonne National Laboratory 2009

Absence Data

Appendix J. Total Number of Calendar Days Absent in Each Diagnostic Category by Gender and Job Category*

\begin{tabular}{|c|c|c|c|c|c|}
\hline & & \multicolumn{4}{|c|}{ Men } \\
\hline & & \multicolumn{3}{|c|}{ Job Category } & \multirow[b]{2}{*}{ TOTAL } \\
\hline & & Security and Fire & Crafts & Line Operators & \\
\hline Diagnostic Category & ICD-9-CM Code & \multirow[b]{2}{*}{0} & \multirow[b]{2}{*}{3} & \multirow[b]{2}{*}{4} & \multirow[b]{2}{*}{47} \\
\hline INFECTIOUS \& PARASITIC DISEASES (DIS) & 001-139 & & & & \\
\hline MALIGNANT NEOPLASMS & $\begin{array}{l}140-208, \\
209.0-209.3 \\
230-234\end{array}$ & 0 & 143 & 0 & 349 \\
\hline BENIGN \& UNCERTAIN NEOPLASMS & $\begin{array}{l}209.4-209.6 \\
210-229,235-239\end{array}$ & 0 & 0 & 0 & 140 \\
\hline ENDOCRINE/METABOLIC/IMMUNITY & $240-279$ & 7 & 173 & 0 & 212 \\
\hline BLOOD \& BLOOD-FORMING ORGANS & $280-289$ & 0 & 49 & 0 & 75 \\
\hline MENTAL DISORDERS & 290-319 & 0 & 217 & 0 & 496 \\
\hline NERVOUS SYSTEM (NS) \& SENSE ORGANS & $320-389$ & 0 & 90 & 7 & 282 \\
\hline CIRCULATORY SYSTEM & $390-459$ & 0 & 280 & 15 & 660 \\
\hline RESPIRATORY SYSTEM & $460-519$ & 9 & 191 & 8 & 515 \\
\hline DIGESTIVE SYSTEM & $520-579$ & 0 & 150 & 0 & 507 \\
\hline GENITOURINARY SYSTEM & $580-629$ & 0 & 68 & 0 & 82 \\
\hline SKIN AND SUBCUTANEOUS TISSUE & $680-709$ & 0 & 12 & 5 & 116 \\
\hline $\begin{array}{l}\text { MUSCULOSKELETAL \& CONNECTIVE } \\
\text { TISSUE }\end{array}$ & $710-739$ & 162 & 511 & 1 & 1,187 \\
\hline $\begin{array}{l}\text { SYMPTOMS, SIGNS, \& ILL-DEFINED } \\
\text { CONDITIONS }\end{array}$ & 780-799 & 10 & 226 & 15 & 421 \\
\hline INJURY \& POISONING & 800-999 & 71 & 395 & 1 & 1,401 \\
\hline
\end{tabular}

\footnotetext{
*Absences with >1 ICD-9-CM code in the same diagnostic category were counted only once. Only those diagnostic categories and gender/job category combinations with at least one occurrence appear in this table.
} 
Argonne National Laboratory 2009

Absence Data

Appendix K. Age-Adjusted Illness and Injury Rates by Diagnostic Category*

Part 1. Men

\begin{tabular}{|c|c|c|c|c|c|}
\hline & & $\begin{array}{l}\text { Number of } \\
\text { Diagnoses }\end{array}$ & $\begin{array}{c}\text { Age-Adjusted } \\
\text { Rate per } 1,000 * *\end{array}$ & $\begin{array}{c}\text { Lower } 95 \% \\
\text { Confidence } \\
\text { Limit per 1,000 }\end{array}$ & $\begin{array}{c}\text { Upper } 95 \% \\
\text { Confidence } \\
\text { Limit per 1,000 }\end{array}$ \\
\hline Diagnostic Category & ICD-9-CM Code & \multirow[b]{2}{*}{10} & \multirow[b]{2}{*}{7.7} & \multirow[b]{2}{*}{3.1} & \multirow[b]{2}{*}{19.5} \\
\hline INFECTIOUS \& PARASITIC DISEASES (DIS) & 001-139 & & & & \\
\hline -Intestinal Infectious Dis & 001-009 & 1 & 0.3 & 0.0 & 2.0 \\
\hline -Other Bacterial Dis & 030-041 & 1 & 0.3 & 0.0 & 2.0 \\
\hline -Polio \& Other Viral CNS Dis & 045-049 & 1 & 0.4 & 0.1 & 3.0 \\
\hline -Other Viral Dis \& Chlamydiae & 070-079 & 7 & 6.8 & 2.4 & 19.2 \\
\hline MALIGNANT NEOPLASMS & $\begin{array}{l}140-208, \\
209.0-209.3 \\
230-234\end{array}$ & 11 & 3.8 & 2.0 & 7.2 \\
\hline -Digestive \& Peritoneal & $150-159$ & 1 & 0.3 & 0.0 & 2.0 \\
\hline -Bone, Connective Tissue, Skin & 170-173, 176 & 2 & 0.8 & 0.2 & 3.3 \\
\hline -Genitourinary & 179-189 & 8 & 2.7 & 1.3 & 5.8 \\
\hline BENIGN \& UNCERTAIN NEOPLASMS & \begin{tabular}{|l|}
$209.4-209.6$ \\
$210-229$, \\
$235-239$
\end{tabular} & 4 & 1.4 & 0.5 & 3.8 \\
\hline ENDOCRINE/METABOLIC/IMMUNITY & $240-279$ & 7 & 4.6 & 1.5 & 14.1 \\
\hline -Other Endocrine Gland Dis & $249-259$ & 3 & 1.0 & 0.3 & 3.1 \\
\hline -Other Metabolic \& Immunity Disorders & 270-279 & 4 & 3.6 & 0.9 & 14.5 \\
\hline BLOOD \& BLOOD-FORMING ORGANS & $280-289$ & 2 & 0.6 & 0.1 & 2.2 \\
\hline MENTAL DISORDERS & 290-319 & 28 & 14.1 & 8.6 & 23.0 \\
\hline -Psychoses & 290-299 & 3 & 1.8 & 0.5 & 6.0 \\
\hline -Non-Psychotic Disorders & $300-302,306-316$ & 18 & 10.2 & 5.5 & 19.0 \\
\hline -Alcohol Dependence & 303 & 4 & 1.3 & 0.5 & 3.4 \\
\hline -Drug Dependence & $304-305$ & 3 & 0.8 & 0.3 & 2.6 \\
\hline NERVOUS SYSTEM (NS) \& SENSE ORGANS & $320-389$ & 17 & 5.4 & 3.3 & 8.8 \\
\hline -Other Disorders of Central NS & 340-349 & 2 & 0.6 & 0.1 & 2.2 \\
\hline -Disorders of Eye & $360-379$ & 7 & 2.6 & 1.3 & 5.6 \\
\hline -Diseases of Ear \& Mastoid & $380-389$ & 8 & 2.2 & 1.1 & 4.4 \\
\hline CIRCULATORY SYSTEM & $390-459$ & 19 & 6.3 & 3.9 & 10.2 \\
\hline -Hypertensive Dis & $401-405$ & 7 & 2.2 & 1.0 & 4.7 \\
\hline -Ischemic Heart Dis & $410-414$ & 7 & 2.2 & 1.0 & 4.8 \\
\hline -Dis of Pulmonary Circulation & $415-417$ & 1 & 0.3 & 0.0 & 2.0 \\
\hline -Other Heart Dis & $420-429$ & 2 & 0.6 & 0.1 & 2.2 \\
\hline -Dis of Arteries \& Capillaries & $440-449$ & 1 & 0.3 & 0.0 & 2.0 \\
\hline -Dis of Veins, Lymphatics, Other & 451-459 & 1 & 0.8 & 0.1 & 5.4 \\
\hline RESPIRATORY SYSTEM & $460-519$ & 88 & 46.1 & 34.1 & 62.4 \\
\hline -Acute Respiratory Infections & $460-466$ & 26 & 15.9 & 8.9 & 28.3 \\
\hline -Other Dis Upper Respiratory Tract & $470-478$ & 13 & 7.2 & 3.3 & 15.8 \\
\hline
\end{tabular}

(Continued)

* Only those diagnostic categories with at least one occurrence appear in this table.

**Standardized to age distribution of 2000 U.S. population. 
Argonne National Laboratory 2009

Absence Data

Appendix K. Age-Adjusted Illness and Injury Rates by Diagnostic Category*

Part 1. Men

\begin{tabular}{|c|c|c|c|c|c|}
\hline & & $\begin{array}{l}\text { Number of } \\
\text { Diagnoses }\end{array}$ & $\begin{array}{c}\text { Age-Adjusted } \\
\text { Rate per } 1,000 * *\end{array}$ & $\begin{array}{c}\text { Lower } 95 \% \\
\text { Confidence } \\
\text { Limit per 1,000 }\end{array}$ & $\begin{array}{c}\text { Upper 95\% } \\
\text { Confidence } \\
\text { Limit per 1,000 }\end{array}$ \\
\hline Diagnostic Category & ICD-9-CM Code & \multirow[b]{2}{*}{20} & \multirow[b]{2}{*}{11.9} & \multirow[b]{2}{*}{6.2} & \multirow[b]{2}{*}{22.7} \\
\hline -Pneumonia \& Influenza & $480-488$ & & & & \\
\hline -Chronic Obstructive Dis & $490-496$ & 26 & 9.8 & 6.5 & 14.8 \\
\hline -Lung Dis from External Agents & $500-508$ & 1 & 0.8 & 0.1 & 5.4 \\
\hline -Other Respiratory Dis & $510-519$ & 2 & 0.6 & 0.1 & 2.2 \\
\hline DIGESTIVE SYSTEM & $520-579$ & 43 & 28.9 & 18.5 & 45.1 \\
\hline -Oral Cavity, Saliva Glands, Jaw & $520-529$ & 5 & 6.4 & 2.1 & 19.4 \\
\hline -Esophagus, Stomach, Duodenum & $530-538$ & 8 & 2.8 & 1.3 & 6.1 \\
\hline -Appendicitis & $540-543$ & 1 & 0.8 & 0.1 & 5.4 \\
\hline -Hernias & $550-553$ & 10 & 5.1 & 1.9 & 14.0 \\
\hline -Enteritis, Colitis & $555-558$ & 9 & 5.9 & 2.3 & 15.1 \\
\hline -Other Intestinal Dis & $560-569$ & 5 & 4.2 & 1.2 & 14.5 \\
\hline -Other Digestive Dis & $570-579$ & 5 & 3.6 & 0.9 & 14.4 \\
\hline GENITOURINARY SYSTEM & $580-629$ & 6 & 2.3 & 1.0 & 5.5 \\
\hline -Other Urinary Dis & $590-599$ & 4 & 1.7 & 0.6 & 5.1 \\
\hline -Male Genital Organ Dis & $600-608$ & 2 & 0.6 & 0.1 & 2.2 \\
\hline SKIN AND SUBCUTANEOUS TISSUE & $680-709$ & 11 & 6.5 & 2.8 & 15.3 \\
\hline -Infections & $680-686$ & 6 & 5.1 & 1.8 & 14.8 \\
\hline -Other Inflammatory Conditions & $690-698$ & 1 & 0.3 & 0.0 & 2.0 \\
\hline -Other & 700-709 & 4 & 1.1 & 0.4 & 3.0 \\
\hline $\begin{array}{l}\text { MUSCULOSKELETAL \& CONNECTIVE } \\
\text { TISSUE }\end{array}$ & $710-739$ & 59 & 24.1 & 17.5 & 33.2 \\
\hline -Arthropathies & $710-719$ & 14 & 5.6 & 3.1 & 10.0 \\
\hline -Dorsopathies & $720-724$ & 31 & 13.9 & 8.6 & 22.3 \\
\hline -Rheumatism, Excluding Back & $725-729$ & 8 & 2.8 & 1.4 & $\overline{5.6}$ \\
\hline -Other Dis \& Acquired Deformities & 730-739 & 6 & 1.8 & 0.8 & 4.1 \\
\hline $\begin{array}{l}\text { SYMPTOMS, SIGNS, \& ILL-DEFINED } \\
\text { CONDITIONS }\end{array}$ & $780-799$ & 46 & 19.8 & 13.0 & 30.2 \\
\hline -Symptoms & 780-789 & 45 & 19.5 & 12.7 & 29.9 \\
\hline -Non-Specific Abnormal Findings & $790-796$ & 1 & 0.3 & 0.0 & 2.0 \\
\hline INJURY \& POISONING & $800-999$ & 53 & 30.9 & 20.4 & 46.9 \\
\hline -Fracture - Skull & 800-804 & 3 & 1.0 & 0.3 & 3.1 \\
\hline -Fracture - Upper Limb & 810-819 & 2 & 0.6 & 0.1 & 2.2 \\
\hline -Fracture - Lower Limb & $820-829$ & 7 & 2.6 & 1.1 & 5.8 \\
\hline -Dislocation & $830-839$ & 10 & 5.1 & 1.9 & 14.0 \\
\hline -Sprains \& Strains - Back & 846-847 & 1 & 0.4 & 0.1 & 3.0 \\
\hline -Sprains \& Strains - Other & $840-845,848$ & 17 & 12.6 & 6.3 & 25.5 \\
\hline
\end{tabular}

(Continued)

*Only those diagnostic categories with at least one occurrence appear in this table.

**Standardized to age distribution of 2000 U.S. population. 
Argonne National Laboratory 2009

Absence Data

Appendix K. Age-Adjusted Illness and Injury Rates by Diagnostic Category*

Part 1. Men

\begin{tabular}{|c|c|c|c|c|c|}
\hline & & $\begin{array}{l}\text { Number of } \\
\text { Diagnoses }\end{array}$ & $\begin{array}{c}\text { Age-Adjusted } \\
\text { Rate per } 1,000 * *\end{array}$ & \begin{tabular}{c|} 
Lower $95 \%$ \\
Confidence \\
Limit per 1,000
\end{tabular} & $\begin{array}{c}\text { Upper } 95 \% \\
\text { Confidence } \\
\text { Limit per } 1,000\end{array}$ \\
\hline Diagnostic Category & ICD-9-CM Code & \multirow[b]{2}{*}{2} & \multirow[b]{2}{*}{0.6} & \multirow[b]{2}{*}{0.1} & \multirow[b]{2}{*}{2.2} \\
\hline -Intracranial Injury & $850-854$ & & & & \\
\hline -Open Wound - Head, Neck, Trunk & $870-879$ & 5 & 4.2 & 1.2 & 14.5 \\
\hline -Late Effects of Accident & 905-909 & 1 & 0.3 & 0.0 & 2.0 \\
\hline -Contusion & $920-924$ & 1 & 0.3 & 0.0 & 2.0 \\
\hline -Burns & $940-949$ & 1 & 0.3 & 0.0 & 2.0 \\
\hline -Complications \& Unspecified Injuries & 958-959 & 2 & 2.8 & 0.5 & 16.2 \\
\hline -Complications of Surgical/Medical Care & 996-999 & 1 & 0.3 & 0.0 & 2.0 \\
\hline Total & & 404 & 202.5 & 175.5 & 233.7 \\
\hline
\end{tabular}

*Only those diagnostic categories with at least one occurrence appear in this table.

**Standardized to age distribution of 2000 U.S. population. 
Argonne National Laboratory 2009

Absence Data

Appendix K. Age-Adjusted Illness and Injury Rates by Diagnostic Category*

Part 2. Women

\begin{tabular}{|c|c|c|c|c|c|}
\hline & & $\begin{array}{l}\text { Number of } \\
\text { Diagnoses }\end{array}$ & $\begin{array}{c}\text { Age-Adjusted } \\
\text { Rate per } 1,000 * *\end{array}$ & \begin{tabular}{|c|} 
Lower $95 \%$ \\
Confidence \\
Limit per 1,000
\end{tabular} & $\begin{array}{c}\text { Upper } 95 \% \\
\text { Confidence } \\
\text { Limit per } 1,000\end{array}$ \\
\hline Diagnostic Category & ICD-9-CM Code & & & & \\
\hline INFECTIOUS \& PARASITIC DISEASES (DIS) & 001-139 & 7 & 6.3 & 3.0 & 13.3 \\
\hline -Intestinal Infectious Dis & 001-009 & 1 & 0.9 & 0.1 & 6.6 \\
\hline -Other Bacterial Dis & 030-041 & 1 & 0.9 & 0.1 & 6.6 \\
\hline -Other Viral Dis \& Chlamydiae & 070-079 & 5 & 4.5 & 1.8 & 10.9 \\
\hline MALIGNANT NEOPLASMS & $\begin{array}{l}140-208, \\
209.0-209.3 \\
230-234\end{array}$ & 4 & 2.3 & 0.9 & 6.2 \\
\hline -Digestive \& Peritoneal & 150-159 & 1 & 0.6 & 0.1 & 4.1 \\
\hline -Breast & 174-175 & 1 & 0.6 & 0.1 & 4.1 \\
\hline -Genitourinary & 179-189 & 1 & 0.6 & 0.1 & 4.1 \\
\hline -Other \& Unspecified Sites & 195-199, 209.7 & 1 & 0.6 & 0.1 & 4.1 \\
\hline BENIGN \& UNCERTAIN NEOPLASMS & $\begin{array}{l}209.4-209.6, \\
210-229,235-239\end{array}$ & 3 & 5.8 & 1.5 & 23.1 \\
\hline ENDOCRINE/METABOLIC/IMMUNITY & $240-279$ & 5 & 7.0 & 2.2 & 22.6 \\
\hline -Other Endocrine Gland Dis & 249-259 & 4 & 6.0 & 1.6 & 22.6 \\
\hline -Other Metabolic \& Immunity Disorders & $270-279$ & 1 & 1.0 & 0.1 & 7.2 \\
\hline MENTAL DISORDERS & 290-319 & 8 & 7.4 & 3.7 & 14.8 \\
\hline -Non-Psychotic Disorders & $300-302,306-316$ & 8 & 7.4 & 3.7 & 14.8 \\
\hline NERVOUS SYSTEM (NS) \& SENSE ORGANS & $320-389$ & 12 & 16.6 & 7.9 & 34.6 \\
\hline -Other Disorders of Central NS & 340-349 & 3 & 6.2 & 1.6 & 23.9 \\
\hline -Disorders of Peripheral NS & 350-359 & 5 & 3.7 & 1.5 & 9.1 \\
\hline -Disorders of Eye & $360-379$ & 2 & 1.9 & 0.5 & 7.8 \\
\hline -Diseases of Ear \& Mastoid & $380-389$ & 2 & 4.8 & 0.9 & 24.3 \\
\hline CIRCULATORY SYSTEM & $390-459$ & 10 & 9.8 & 4.1 & 23.4 \\
\hline -Hypertensive Dis & $401-405$ & 2 & 1.2 & 0.3 & 4.6 \\
\hline -Ischemic Heart Dis & $410-414$ & 2 & 1.2 & 0.3 & 4.6 \\
\hline -Other Heart Dis & $420-429$ & 2 & 4.8 & 0.9 & 24.3 \\
\hline -Cerebrovascular Dis & $430-438$ & 2 & 1.5 & 0.4 & 6.2 \\
\hline -Dis of Veins, Lymphatics, Other & $451-459$ & 2 & 1.2 & 0.3 & $\overline{4.6}$ \\
\hline RESPIRATORY SYSTEM & $460-519$ & 63 & 69.9 & 51.2 & 95.4 \\
\hline -Acute Respiratory Infections & $460-466$ & 18 & 18.6 & 10.7 & 32.6 \\
\hline -Other Dis Upper Respiratory Tract & $470-478$ & 6 & 11.2 & 4.1 & 30.6 \\
\hline -Pneumonia \& Influenza & $480-488$ & 19 & 22.4 & 12.5 & 40.2 \\
\hline -Chronic Obstructive Dis & $490-496$ & 18 & 16.0 & 9.9 & 26.0 \\
\hline -Other Respiratory Dis & $510-519$ & 2 & 1.6 & 0.4 & 6.7 \\
\hline DIGESTIVE SYSTEM & $520-579$ & 17 & 17.6 & 9.7 & 32.1 \\
\hline
\end{tabular}

(Continued)

* Only those diagnostic categories with at least one occurrence appear in this table.

**Standardized to age distribution of 2000 U.S. population. 
Argonne National Laboratory 2009

Absence Data

Appendix K. Age-Adjusted Illness and Injury Rates by Diagnostic Category*

Part 2. Women

\begin{tabular}{|c|c|c|c|c|c|}
\hline & & $\begin{array}{l}\text { Number of } \\
\text { Diagnoses }\end{array}$ & $\begin{array}{c}\text { Age-Adjusted } \\
\text { Rate per } 1,000 * *\end{array}$ & \begin{tabular}{|c|} 
Lower $95 \%$ \\
Confidence \\
Limit per 1,000
\end{tabular} & $\begin{array}{c}\text { Upper } 95 \% \\
\text { Confidence } \\
\text { Limit per } \mathbf{1 , 0 0 0}\end{array}$ \\
\hline Diagnostic Category & ICD-9-CM Code & \multirow[b]{2}{*}{5} & \multirow[b]{2}{*}{4.9} & \multirow[b]{2}{*}{1.9} & \multirow[b]{2}{*}{12.6} \\
\hline -Oral Cavity, Saliva Glands, Jaw & $520-529$ & & & & \\
\hline -Esophagus, Stomach, Duodenum & $530-538$ & 4 & 2.7 & 1.0 & 7.3 \\
\hline -Hernias & $550-553$ & 1 & 1.8 & 0.2 & 12.4 \\
\hline -Enteritis, Colitis & $555-558$ & 2 & 1.5 & 0.4 & 6.2 \\
\hline -Other Intestinal Dis & $560-569$ & 2 & 1.5 & 0.4 & 6.2 \\
\hline -Other Digestive Dis & $570-579$ & 3 & 5.4 & 1.2 & 23.2 \\
\hline GENITOURINARY SYSTEM & $580-629$ & 10 & 14.5 & 6.5 & 32.6 \\
\hline -Other Urinary Dis & $590-599$ & 2 & 4.4 & 0.8 & 24.8 \\
\hline -Pelvic Inflammatory Dis & 614-616 & 1 & 3.9 & 0.5 & 27.3 \\
\hline -Other Female Disorders & $617-629$ & 7 & 6.2 & 2.9 & 13.3 \\
\hline PREGNANCY \& CHILDBIRTH & $630-679$ & 2 & 5.6 & 1.3 & 24.6 \\
\hline -Pregnancy with Abortive Outcome & 634-639 & 2 & 5.6 & 1.3 & 24.6 \\
\hline SKIN AND SUBCUTANEOUS TISSUE & 680-709 & 1 & 1.0 & 0.1 & 7.2 \\
\hline -Infections & $680-686$ & 1 & 1.0 & 0.1 & 7.2 \\
\hline $\begin{array}{l}\text { MUSCULOSKELETAL \& CONNECTIVE } \\
\text { TISSUE }\end{array}$ & 710-739 & 28 & 23.2 & 15.7 & 34.2 \\
\hline -Arthropathies & 710-719 & 10 & 7.1 & 3.7 & 13.5 \\
\hline -Dorsopathies & $720-724$ & 11 & 9.8 & 5.4 & 17.8 \\
\hline -Rheumatism, Excluding Back & 725-729 & 2 & 1.5 & 0.4 & 6.2 \\
\hline -Other Dis \& Acquired Deformities & 730-739 & 5 & 4.9 & 1.9 & $\overline{12.6}$ \\
\hline CONGENITAL ANOMALIES & $740-759$ & 3 & 2.4 & 0.8 & 7.7 \\
\hline $\begin{array}{l}\text { SYMPTOMS, SIGNS, \& ILL-DEFINED } \\
\text { CONDITIONS }\end{array}$ & 780-799 & 30 & 43.1 & 26.9 & 69.2 \\
\hline -Symptoms & $780-789$ & 28 & 38.7 & 23.7 & 63.1 \\
\hline -Non-Specific Abnormal Findings & $790-796$ & 2 & 4.4 & 0.8 & 24.8 \\
\hline INJURY \& POISONING & $800-999$ & 21 & 18.1 & 11.6 & 28.3 \\
\hline -Fracture - Upper Limb & 810-819 & 1 & 0.9 & 0.1 & 6.6 \\
\hline -Fracture - Lower Limb & $820-829$ & 3 & 1.7 & 0.6 & 5.4 \\
\hline -Dislocation & 830-839 & 3 & 2.5 & 0.8 & 8.0 \\
\hline -Sprains \& Strains - Other & $840-845,848$ & 4 & 3.4 & 1.3 & 9.3 \\
\hline -Late Effects of Accident & 905-909 & 2 & 1.8 & 0.5 & 7.4 \\
\hline -Superficial Injury & 910-919 & 1 & 0.9 & 0.1 & 6.6 \\
\hline -Contusion & $920-924$ & 1 & 0.6 & 0.1 & 4.1 \\
\hline -Complications \& Unspecified Injuries & 958-959 & 5 & 5.1 & 2.0 & 13.0 \\
\hline -Complications of Surgical/Medical Care & 996-999 & 1 & 1.0 & 0.1 & 7.2 \\
\hline Total & & 224 & 250.8 & 212.0 & 296.6 \\
\hline
\end{tabular}

* Only those diagnostic categories with at least one occurrence appear in this table.

**Standardized to age distribution of 2000 U.S. population. 
Argonne National Laboratory 2009

Absence Data

Appendix K. Age-Adjusted Illness and Injury Rates by Diagnostic Category*

Part 3. Men and Women

\begin{tabular}{|c|c|c|c|c|c|}
\hline & & $\begin{array}{l}\text { Number of } \\
\text { Diagnoses }\end{array}$ & $\begin{array}{c}\text { Age-Adjusted } \\
\text { Rate per 1,000** }\end{array}$ & \begin{tabular}{|c|} 
Lower $95 \%$ \\
Confidence \\
Limit per 1,000
\end{tabular} & $\begin{array}{c}\text { Upper } 95 \% \\
\text { Confidence } \\
\text { Limit per } 1,000\end{array}$ \\
\hline Diagnostic Category & ICD-9-CM Code & & & & \\
\hline INFECTIOUS \& PARASITIC DISEASES (DIS) & 001-139 & 17 & 6.7 & 3.4 & 13.3 \\
\hline -Intestinal Infectious Dis & 001-009 & 2 & 0.5 & 0.1 & 2.0 \\
\hline -Other Bacterial Dis & 030-041 & 2 & 0.5 & 0.1 & 2.0 \\
\hline -Polio \& Other Viral CNS Dis & 045-049 & 1 & 0.3 & 0.0 & 2.0 \\
\hline -Other Viral Dis \& Chlamydiae & 070-079 & 12 & 5.5 & 2.4 & 12.3 \\
\hline MALIGNANT NEOPLASMS & $\begin{array}{l}140-208, \\
209.0-209.3, \\
230-234\end{array}$ & 15 & 3.6 & 2.1 & 6.1 \\
\hline -Digestive \& Peritoneal & $150-159$ & 2 & 0.4 & 0.1 & 1.5 \\
\hline -Bone, Connective Tissue, Skin & 170-173, 176 & 2 & 0.6 & 0.1 & 2.3 \\
\hline -Breast & 174-175 & 1 & 0.2 & 0.0 & 1.3 \\
\hline -Genitourinary & 179-189 & 9 & 2.2 & 1.1 & 4.5 \\
\hline -Other \& Unspecified Sites & 195-199, 209.7 & 1 & 0.2 & 0.0 & $\overline{1.3}$ \\
\hline BENIGN \& UNCERTAIN NEOPLASMS & $\begin{array}{l}\text { 209.4-209.6, } \\
210-229,235-239\end{array}$ & 7 & 3.0 & 1.0 & 8.7 \\
\hline ENDOCRINE/METABOLIC/IMMUNITY & $240-279$ & 12 & 5.3 & 2.3 & 12.2 \\
\hline -Other Endocrine Gland Dis & 249-259 & 7 & 2.8 & 0.9 & 8.5 \\
\hline -Other Metabolic \& Immunity Disorders & 270-279 & 5 & 2.5 & 0.7 & 8.6 \\
\hline BLOOD \& BLOOD-FORMING ORGANS & $280-289$ & 2 & 0.4 & 0.1 & 1.7 \\
\hline MENTAL DISORDERS & 290-319 & 36 & 11.9 & 7.9 & 17.8 \\
\hline -Psychoses & 290-299 & 3 & 1.2 & 0.4 & 4.2 \\
\hline -Non-Psychotic Disorders & $300-302,306-316$ & 26 & 9.2 & 5.7 & 14.9 \\
\hline -Alcohol Dependence & 303 & 4 & 0.9 & 0.3 & 2.3 \\
\hline -Drug Dependence & 304-305 & 3 & 0.6 & 0.2 & 1.8 \\
\hline NERVOUS SYSTEM (NS) \& SENSE ORGANS & 320-389 & 29 & 9.4 & 5.6 & 15.8 \\
\hline -Other Disorders of Central NS & 340-349 & 5 & 2.6 & 0.8 & 8.8 \\
\hline -Disorders of Peripheral NS & 350-359 & 5 & 1.1 & 0.4 & 2.6 \\
\hline -Disorders of Eye & $360-379$ & 9 & 2.4 & 1.2 & 4.6 \\
\hline -Diseases of Ear \& Mastoid & 380-389 & 10 & 3.4 & 1.3 & 8.7 \\
\hline CIRCULATORY SYSTEM & $390-459$ & 29 & 7.9 & 4.9 & 12.9 \\
\hline -Hypertensive Dis & $401-405$ & 9 & 1.9 & 1.0 & 3.7 \\
\hline -Ischemic Heart Dis & $410-414$ & 9 & 2.0 & 1.1 & 4.0 \\
\hline -Dis of Pulmonary Circulation & $415-417$ & 1 & 0.2 & 0.0 & 1.3 \\
\hline -Other Heart Dis & $420-429$ & 4 & 2.2 & 0.6 & 8.8 \\
\hline -Cerebrovascular Dis & $430-438$ & 2 & 0.5 & 0.1 & 2.0 \\
\hline -Dis of Arteries \& Capillaries & $440-449$ & 1 & 0.2 & 0.0 & 1.6 \\
\hline
\end{tabular}

(Continued)

* Only those diagnostic categories with at least one occurrence appear in this table.

**Standardized to age distribution of 2000 U.S. population. 
Argonne National Laboratory 2009

Absence Data

Appendix K. Age-Adjusted Illness and Injury Rates by Diagnostic Category*

Part 3. Men and Women

\begin{tabular}{|c|c|c|c|c|c|}
\hline & & $\begin{array}{c}\text { Number of } \\
\text { Diagnoses }\end{array}$ & $\begin{array}{c}\text { Age-Adjusted } \\
\text { Rate per 1,000** }\end{array}$ & $\begin{array}{c}\text { Lower } 95 \% \\
\text { Confidence } \\
\text { Limit per } 1,000\end{array}$ & $\begin{array}{c}\text { Upper } 95 \% \\
\text { Confidence } \\
\text { Limit per } \mathbf{1 , 0 0 0}\end{array}$ \\
\hline Diagnostic Category & ICD-9-CM Code & \multirow[b]{2}{*}{3} & \multirow[b]{2}{*}{0.9} & \multirow[b]{2}{*}{0.3} & \multirow[b]{2}{*}{3.3} \\
\hline -Dis of Veins, Lymphatics, Other & 451-459 & & & & \\
\hline RESPIRATORY SYSTEM & $460-519$ & 151 & 52.7 & 42.2 & 65.7 \\
\hline -Acute Respiratory Infections & $460-466$ & 44 & 16.1 & 10.6 & 24.5 \\
\hline -Other Dis Upper Respiratory Tract & $470-478$ & 19 & 8.8 & 4.7 & 16.5 \\
\hline -Pneumonia \& Influenza & $480-488$ & 39 & 15.3 & 9.8 & 23.8 \\
\hline -Chronic Obstructive Dis & $490-496$ & 44 & 11.2 & 8.2 & 15.3 \\
\hline -Lung Dis from External Agents & $500-508$ & 1 & 0.5 & 0.1 & 3.7 \\
\hline -Other Respiratory Dis & $510-519$ & 4 & 0.8 & 0.3 & 2.1 \\
\hline DIGESTIVE SYSTEM & $520-579$ & 60 & 24.7 & 17.3 & 35.3 \\
\hline -Oral Cavity, Saliva Glands, Jaw & $520-529$ & 10 & 5.5 & 2.4 & 12.6 \\
\hline -Esophagus, Stomach, Duodenum & $530-538$ & 12 & 2.9 & 1.6 & 5.3 \\
\hline -Appendicitis & $540-543$ & 1 & 0.5 & 0.1 & 3.7 \\
\hline -Hernias & $550-553$ & 11 & 3.9 & 1.7 & 9.2 \\
\hline -Enteritis, Colitis & $555-558$ & 11 & 4.4 & 1.9 & 9.8 \\
\hline -Other Intestinal Dis & $560-569$ & 7 & 3.2 & 1.2 & 9.0 \\
\hline -Other Digestive Dis & $570-579$ & 8 & 4.3 & 1.6 & 11.7 \\
\hline GENITOURINARY SYSTEM & $580-629$ & 16 & 6.4 & 3.1 & 13.1 \\
\hline -Other Urinary Dis & $590-599$ & 6 & 3.0 & 1.0 & 8.9 \\
\hline -Male Genital Organ Dis & $600-608$ & 2 & 0.5 & 0.1 & 1.8 \\
\hline -Pelvic Inflammatory Dis & 614-616 & 1 & 1.5 & 0.2 & 10.6 \\
\hline -Other Female Disorders & $617-629$ & 7 & 1.5 & 0.7 & 3.2 \\
\hline PREGNANCY \& CHILDBIRTH & $630-679$ & 2 & 2.0 & 0.4 & 9.4 \\
\hline -Pregnancy with Abortive Outcome & $634-639$ & 2 & 2.0 & 0.4 & 9.4 \\
\hline SKIN AND SUBCUTANEOUS TISSUE & $680-709$ & 12 & 4.6 & 2.1 & 9.9 \\
\hline -Infections & $680-686$ & 7 & 3.5 & 1.3 & 9.3 \\
\hline -Other Inflammatory Conditions & 690-698 & 1 & 0.2 & 0.0 & 1.3 \\
\hline -Other & 700-709 & 4 & 0.9 & 0.3 & 2.3 \\
\hline $\begin{array}{l}\text { MUSCULOSKELETAL \& CONNECTIVE } \\
\text { TISSUE }\end{array}$ & $710-739$ & 87 & 23.7 & 18.5 & 30.2 \\
\hline -Arthropathies & $710-719$ & 24 & 6.0 & 3.9 & 9.3 \\
\hline -Dorsopathies & $720-724$ & 42 & 12.5 & 8.6 & 18.1 \\
\hline -Rheumatism, Excluding Back & $725-729$ & 10 & 2.4 & 1.3 & 4.6 \\
\hline -Other Dis \& Acquired Deformities & $730-739$ & 11 & 2.7 & 1.4 & 5.1 \\
\hline CONGENITAL ANOMALIES & 740-759 & 3 & 0.8 & 0.2 & 2.4 \\
\hline $\begin{array}{l}\text { SYMPTOMS, SIGNS, \& ILL-DEFINED } \\
\text { CONDITIONS }\end{array}$ & $780-799$ & 76 & 28.5 & 20.6 & 39.5 \\
\hline
\end{tabular}

(Continued)

*Only those diagnostic categories with at least one occurrence appear in this table.

**Standardized to age distribution of 2000 U.S. population. 
Argonne National Laboratory 2009

Absence Data

Appendix K. Age-Adjusted Illness and Injury Rates by Diagnostic Category*

Part 3. Men and Women

\begin{tabular}{|c|c|c|c|c|c|}
\hline & & $\begin{array}{l}\text { Number of } \\
\text { Diagnoses }\end{array}$ & $\begin{array}{c}\text { Age-Adjusted } \\
\text { Rate per 1,000** }\end{array}$ & $\begin{array}{c}\text { Lower } 95 \% \\
\text { Confidence } \\
\text { Limit per 1,000 }\end{array}$ & $\begin{array}{c}\text { Upper } 95 \% \\
\text { Confidence } \\
\text { Limit per 1,000 }\end{array}$ \\
\hline Diagnostic Category & ICD-9-CM Code & \multirow[b]{2}{*}{73} & \multirow[b]{2}{*}{26.6} & \multirow[b]{2}{*}{19.1} & \multirow[b]{2}{*}{37.1} \\
\hline -Symptoms & 780-789 & & & & \\
\hline -Non-Specific Abnormal Findings & $790-796$ & 3 & 1.9 & 0.4 & 9.1 \\
\hline INJURY \& POISONING & 800-999 & 74 & 25.8 & 18.7 & 35.6 \\
\hline -Fracture - Skull & $800-804$ & 3 & 0.7 & 0.2 & 2.3 \\
\hline -Fracture - Upper Limb & $810-819$ & 3 & 0.7 & 0.2 & 2.2 \\
\hline -Fracture - Lower Limb & $820-829$ & 10 & 2.4 & 1.2 & 4.7 \\
\hline -Dislocation & $830-839$ & 13 & 4.0 & 1.8 & 9.1 \\
\hline -Sprains \& Strains - Back & 846-847 & 1 & 0.3 & 0.0 & 2.0 \\
\hline -Sprains \& Strains - Other & $840-845,848$ & 21 & 9.1 & 5.0 & 16.8 \\
\hline -Intracranial Injury & $850-854$ & 2 & 0.4 & 0.1 & 1.5 \\
\hline -Open Wound - Head, Neck, Trunk & $870-879$ & 5 & 2.7 & 0.8 & 8.9 \\
\hline -Late Effects of Accident & 905-909 & 3 & 0.8 & 0.2 & 2.4 \\
\hline -Superficial Injury & $910-919$ & 1 & 0.3 & 0.0 & 2.0 \\
\hline -Contusion & $920-924$ & 2 & 0.4 & 0.1 & 1.7 \\
\hline -Burns & 940-949 & 1 & 0.2 & 0.0 & 1.3 \\
\hline -Complications \& Unspecified Injuries & 958-959 & 7 & 3.3 & 1.2 & 9.0 \\
\hline -Complications of Surgical/Medical Care & 996-999 & 2 & 0.4 & 0.1 & 1.7 \\
\hline Total & & 628 & 217.4 & 194.9 & 242.5 \\
\hline
\end{tabular}

* Only those diagnostic categories with at least one occurrence appear in this table.

**Standardized to age distribution of 2000 U.S. population. 
Appendix L. Number of Workers with at Least One OSHA Event by Gender, Age, and Job Category*

\begin{tabular}{|c|c|c|c|c|c|c|c|c|c|}
\hline \multirow{3}{*}{ Job Category } & \multicolumn{3}{|c|}{ Women } & \multicolumn{5}{|c|}{ Men } & \multirow[b]{3}{*}{ TOTAL } \\
\hline & \multicolumn{2}{|c|}{ Age Group } & \multirow[b]{2}{*}{ TOTAL } & \multicolumn{4}{|c|}{ Age Group } & \multirow[b]{2}{*}{ TOTAL } & \\
\hline & $40-49$ & $50+$ & & $16-29$ & $30-39$ & $40-49$ & $50+$ & & \\
\hline Professional & 1 & 0 & 1 & 0 & 0 & 0 & 4 & 4 & 5 \\
\hline Administrative Support & 0 & 2 & 2 & 0 & 0 & 1 & 1 & 2 & 4 \\
\hline Service & 0 & 2 & 2 & 2 & 0 & 2 & 3 & 7 & 9 \\
\hline Security and Fire & 1 & 0 & 1 & 0 & 0 & 1 & 1 & 2 & 3 \\
\hline Crafts & 0 & 0 & 0 & 1 & 0 & 1 & 4 & 6 & 6 \\
\hline Line Operators & 1 & 2 & 3 & 0 & 1 & 0 & 0 & 1 & 4 \\
\hline TOTAL & 3 & 6 & 9 & 3 & 1 & 5 & 13 & 22 & 31 \\
\hline
\end{tabular}

*Only those job categories and gender/age combinations with at least one OSHA event appear in this table.

Appendix M. Total Number of Workdays Lost or with Restricted Activity from OSHA Events by Gender and Age

\begin{tabular}{|c|c|c|c|c|c|c|c|c|c|}
\hline \multirow[b]{2}{*}{ Age Group } & \multicolumn{3}{|c|}{ Women } & \multicolumn{3}{|c|}{ Men } & \multicolumn{3}{|c|}{ TOTAL } \\
\hline & $\begin{array}{c}\text { Number of } \\
\text { Events }\end{array}$ & \begin{tabular}{|c} 
Days \\
Restricted
\end{tabular} & Days Lost & $\begin{array}{c}\text { Number of } \\
\text { Events }\end{array}$ & \begin{tabular}{|c|} 
Days \\
Restricted
\end{tabular} & Days Lost & \begin{tabular}{|c|} 
Number of \\
Events
\end{tabular} & $\begin{array}{c}\text { Days } \\
\text { Restricted }\end{array}$ & Days Lost \\
\hline $16-29$ & 0 & 0 & 0 & 3 & 3 & 1 & 3 & 3 & 1 \\
\hline $30-39$ & 0 & 0 & 0 & 1 & 0 & 0 & 1 & 0 & 0 \\
\hline $40-49$ & 3 & 0 & 0 & 5 & 76 & 0 & 8 & 76 & 0 \\
\hline $50+$ & 6 & 14 & 293 & 13 & 187 & 33 & 19 & 201 & 326 \\
\hline TOTAL & 9 & 14 & 293 & 22 & 266 & 34 & 31 & 280 & 327 \\
\hline
\end{tabular}

Appendix N. Total Number of Workdays Lost or with Restricted Activity from OSHA Events by Gender and Job Category*

\begin{tabular}{|c|c|c|c|c|c|c|c|c|c|}
\hline Job Category & \multicolumn{3}{|c|}{ Women } & \multicolumn{3}{|c|}{ Men } & \multicolumn{3}{|c|}{ TOTAL } \\
\hline $\begin{array}{l}\text { Administrative } \\
\text { Support }\end{array}$ & 2 & 0 & 3 & 2 & 26 & 0 & 4 & 26 & 3 \\
\hline Crafts & 0 & 0 & 0 & 6 & 41 & 12 & 6 & 41 & 12 \\
\hline Line Operators & 3 & 4 & 0 & 1 & 0 & 0 & 4 & 4 & 0 \\
\hline TOTAL & 9 & 14 & 293 & 22 & 266 & 34 & 31 & 280 & 327 \\
\hline
\end{tabular}

*Only those job categories with at least one OSHA event appear in this table. 
Argonne National Laboratory 2009

OSHA Data

Appendix O. Number of Diagnoses in Each Diagnostic Category by Gender and Age*

\begin{tabular}{|c|c|c|c|c|}
\hline & & \multicolumn{3}{|c|}{ Women } \\
\hline & & \multicolumn{2}{|c|}{ Age Group } & \multirow[b]{2}{*}{ TOTAI } \\
\hline & & $40-49$ & $50+$ & \\
\hline Diagnostic Category & ICD-9-CM code & \multirow[b]{2}{*}{0} & \multirow[b]{2}{*}{1} & \multirow[b]{2}{*}{1} \\
\hline NERVOUS SYSTEM (NS) \& SENSE ORGANS & $320-389$ & & & \\
\hline -Disorders of Peripheral NS & $350-359$ & 0 & 1 & 1 \\
\hline RESPIRATORY SYSTEM & $460-519$ & 1 & 0 & 1 \\
\hline -Lung Dis from External Agents & $500-508$ & 1 & 0 & 1 \\
\hline $\begin{array}{l}\text { MUSCULOSKELETAL \& CONNECTIVE } \\
\text { TISSUE }\end{array}$ & $710-739$ & 2 & 2 & 4 \\
\hline -Arthropathies & 710-719 & 2 & 1 & 3 \\
\hline -Dorsopathies & $720-724$ & 0 & 0 & 0 \\
\hline -Rheumatism, Excluding Back & 725-729 & 0 & 1 & 1 \\
\hline $\begin{array}{l}\text { SYMPTOMS, SIGNS, \& ILL-DEFINED } \\
\text { CONDITIONS }\end{array}$ & $780-799$ & 0 & 0 & 0 \\
\hline -Symptoms & 780-789 & 0 & 0 & 0 \\
\hline INJURY \& POISONING & 800-999 & 3 & 5 & 8 \\
\hline -Fracture - Upper Limb & $810-819$ & 0 & 0 & 0 \\
\hline -Fracture - Lower Limb & $820-829$ & 0 & 1 & 1 \\
\hline -Sprains \& Strains - Back & 846-847 & 0 & 1 & 1 \\
\hline -Sprains \& Strains - Other & $840-845,848$ & 0 & 2 & 2 \\
\hline -Intracranial Injury & $850-854$ & 0 & 0 & 0 \\
\hline -Open Wound - Upper Limb & 880-887 & 0 & 0 & 0 \\
\hline -Contusion & 920-924 & 0 & 0 & 0 \\
\hline -Foreign Body Entering Orifice & 930-939 & 0 & 0 & 0 \\
\hline -Complications \& Unspecified Injuries & 958-959 & 2 & 1 & 3 \\
\hline -Toxic Effects - Non-medicinal & $980-989$ & 1 & 0 & 1 \\
\hline
\end{tabular}

*Only those diagnostic categories and gender/age combinations with at least one OSHA event appear in this table. 


\section{Argonne National Laboratory 2009}

\section{OSHA Data}

Appendix O. Number of Diagnoses in Each Diagnostic Category by Gender and Age*

\begin{tabular}{|c|c|c|c|c|c|c|c|}
\hline & & \multicolumn{5}{|c|}{ Men } & \multirow[b]{3}{*}{ TOTAL } \\
\hline & & \multicolumn{4}{|c|}{ Age Group } & \multirow[b]{2}{*}{ TOTAL } & \\
\hline & & $16-29$ & 30 - 39 & $40-49$ & $\mathbf{5 0 +}$ & & \\
\hline Diagnostic Category & ICD-9-CM code & & & & & & \\
\hline NERVOUS SYSTEM (NS) \& SENSE ORGANS & $320-389$ & 0 & 0 & 0 & 0 & 0 & 1 \\
\hline -Disorders of Peripheral NS & $350-359$ & 0 & 0 & 0 & 0 & 0 & 1 \\
\hline RESPIRATORY SYSTEM & $460-519$ & 0 & 0 & 0 & 0 & 0 & 1 \\
\hline -Lung Dis from External Agents & $500-508$ & 0 & 0 & 0 & 0 & 0 & 1 \\
\hline $\begin{array}{l}\text { MUSCULOSKELETAL \& CONNECTIVE } \\
\text { TISSUE }\end{array}$ & 710-739 & 1 & 0 & 0 & 5 & 6 & 10 \\
\hline -Arthropathies & 710-719 & 0 & 0 & 0 & 4 & 4 & 7 \\
\hline -Dorsopathies & $720-724$ & 1 & 0 & 0 & 0 & 1 & 1 \\
\hline -Rheumatism, Excluding Back & $725-729$ & 0 & 0 & 0 & 1 & 1 & 2 \\
\hline $\begin{array}{l}\text { SYMPTOMS, SIGNS, \& ILL-DEFINED } \\
\text { CONDITIONS }\end{array}$ & 780-799 & 1 & 0 & 0 & 0 & 1 & 1 \\
\hline -Symptoms & $780-789$ & 1 & 0 & 0 & 0 & 1 & 1 \\
\hline INJURY \& POISONING & 800-999 & 3 & 1 & 5 & 13 & 22 & 30 \\
\hline -Fracture - Upper Limb & 810-819 & 0 & 0 & 1 & 1 & 2 & 2 \\
\hline -Fracture - Lower Limb & $820-829$ & 0 & 0 & 0 & 1 & 1 & 2 \\
\hline -Sprains \& Strains - Back & 846-847 & 0 & 0 & 0 & 0 & 0 & 1 \\
\hline -Sprains \& Strains - Other & $840-845,848$ & 1 & 0 & 1 & 6 & 8 & 10 \\
\hline -Intracranial Injury & $850-854$ & 0 & 0 & 0 & 1 & 1 & 1 \\
\hline -Open Wound - Upper Limb & $880-887$ & 1 & 1 & 2 & 1 & 5 & 5 \\
\hline -Contusion & 920-924 & 0 & 0 & 0 & 1 & 1 & 1 \\
\hline -Foreign Body Entering Orifice & 930-939 & 0 & 0 & 1 & 0 & 1 & 1 \\
\hline -Complications \& Unspecified Injuries & 958-959 & 1 & 0 & 0 & 2 & 3 & 6 \\
\hline -Toxic Effects - Non-medicinal & $980-989$ & 0 & 0 & 0 & 0 & 0 & 1 \\
\hline
\end{tabular}

\begin{tabular}{|c|c|c|c|c|c|c|c|c|c|}
\hline & \multicolumn{3}{|c|}{ Women } & \multicolumn{5}{|c|}{ Men } & \multirow[b]{3}{*}{ TOTAL } \\
\hline & \multicolumn{2}{|c|}{ Age Group } & \multirow[b]{2}{*}{ TOTAL } & \multicolumn{4}{|c|}{ Age Group } & \multirow[b]{2}{*}{ TOTAL } & \\
\hline & $40-49$ & $50+$ & & $16-29$ & 30 - 39 & $40-49$ & $50+$ & & \\
\hline Diagnostic Category & \multirow[b]{2}{*}{6} & \multirow[b]{2}{*}{8} & \multirow[b]{2}{*}{14} & \multirow[b]{2}{*}{5} & \multirow[b]{2}{*}{1} & \multirow[b]{2}{*}{5} & \multirow[b]{2}{*}{18} & \multirow[b]{2}{*}{29} & \multirow[b]{2}{*}{43} \\
\hline Total & & & & & & & & & \\
\hline
\end{tabular}

*Only those diagnostic categories and gender/age combinations with at least one OSHA event appear in this table. 


\section{Argonne National Laboratory 2009}

\section{OSHA Data}

Appendix P. Number of Workdays Lost or with Restricted Activity in Each Diagnostic Category by Gender and Age*

\begin{tabular}{|c|c|c|c|c|c|}
\hline & & \multicolumn{4}{|c|}{ Women } \\
\hline & & \multicolumn{4}{|c|}{ Age Group } \\
\hline & & \multicolumn{2}{|c|}{$40-49$} & \multicolumn{2}{|c|}{$50+$} \\
\hline & & \begin{tabular}{|c|} 
Days \\
Restricted
\end{tabular} & Days Lost & \begin{tabular}{|c|} 
Days \\
Restricted
\end{tabular} & Days Lost \\
\hline Diagnostic Category & ICD-9-CM Codes & \multirow[b]{2}{*}{0} & \multirow[b]{2}{*}{0} & \multirow[b]{2}{*}{2} & \multirow[b]{2}{*}{180} \\
\hline -Disorders of Peripheral NS & $350-359$ & & & & \\
\hline -Lung Dis from External Agents & $500-508$ & 0 & 0 & 0 & 0 \\
\hline -Arthropathies & $710-719$ & 0 & 0 & 2 & 180 \\
\hline -Rheumatism, Excluding Back & 725-729 & 0 & 0 & 2 & 180 \\
\hline -Fracture - Lower Limb & $820-829$ & 0 & 0 & 0 & 0 \\
\hline -Sprains \& Strains - Back & 846-847 & 0 & 0 & 0 & 3 \\
\hline -Sprains \& Strains - Other & $840-845,848$ & 0 & 0 & 12 & 110 \\
\hline $\begin{array}{l}\text {-Complications \& Unspecified } \\
\text { Injuries }\end{array}$ & 958-959 & 0 & 0 & 0 & 0 \\
\hline -Toxic Effects - Non-medicinal & 980-989 & 0 & 0 & 0 & 0 \\
\hline
\end{tabular}

\begin{tabular}{|c|c|c|c|c|c|c|c|c|c|}
\hline & & \multicolumn{8}{|c|}{ Men } \\
\hline & & \multicolumn{8}{|c|}{ Age Group } \\
\hline & & \multicolumn{2}{|c|}{$16-29$} & \multicolumn{2}{|c|}{$30-39$} & \multicolumn{2}{|c|}{$40-49$} & \multicolumn{2}{|c|}{$50+$} \\
\hline & & $\begin{array}{c}\text { Days } \\
\text { Restricted }\end{array}$ & $\begin{array}{l}\text { Days } \\
\text { Lost }\end{array}$ & $\begin{array}{c}\text { Days } \\
\text { Restricted }\end{array}$ & $\begin{array}{l}\text { Days } \\
\text { Lost }\end{array}$ & \begin{tabular}{|c|} 
Days \\
Restricted
\end{tabular} & $\begin{array}{l}\text { Days } \\
\text { Lost }\end{array}$ & $\begin{array}{c}\text { Days } \\
\text { Restricted }\end{array}$ & $\begin{array}{l}\text { Days } \\
\text { Lost }\end{array}$ \\
\hline Diagnostic Category & ICD-9-CM Codes & & & & & & & & \\
\hline -Arthropathies & $710-719$ & 0 & 0 & 0 & 0 & 0 & 0 & 44 & 3 \\
\hline -Dorsopathies & $720-724$ & 2 & 0 & 0 & 0 & 0 & 0 & 0 & 0 \\
\hline -Rheumatism, Excluding Back & 725-729 & 0 & 0 & 0 & 0 & 0 & 0 & 28 & 3 \\
\hline -Symptoms & 780-789 & 0 & 1 & 0 & 0 & 0 & 0 & 0 & 0 \\
\hline -Fracture - Upper Limb & 810-819 & 0 & 0 & 0 & 0 & 56 & 0 & 25 & 0 \\
\hline -Fracture - Lower Limb & $820-829$ & 0 & 0 & 0 & 0 & 0 & 0 & 39 & 0 \\
\hline -Sprains \& Strains - Other & $840-845,848$ & 1 & 0 & 0 & 0 & 0 & 0 & 38 & 3 \\
\hline -Intracranial Injury & $850-854$ & 0 & 0 & 0 & 0 & 0 & 0 & 23 & 21 \\
\hline -Open Wound - Upper Limb & $880-887$ & 0 & 1 & 0 & 0 & 20 & 0 & 27 & 0 \\
\hline -Contusion & 920-924 & 0 & 0 & 0 & 0 & 0 & 0 & 0 & 0 \\
\hline -Foreign Body Entering Orifice & 930-939 & 0 & 0 & 0 & 0 & 0 & 0 & 0 & 0 \\
\hline $\begin{array}{l}\text {-Complications \& Unspecified } \\
\text { Injuries }\end{array}$ & 958-959 & 2 & 0 & 0 & 0 & 0 & 0 & 29 & 9 \\
\hline
\end{tabular}

*OSHA events with >1 ICD-9-CM code in the same diagnostic category were counted only once. Only those diagnostic categories and gender/age combinations with at least one occurrence appear in this table. 
Argonne National Laboratory 2009

OSHA Data

Appendix Q. Number of Occurrences in Each Accident Category by Gender and Age*

\begin{tabular}{|c|c|c|c|c|c|c|c|c|c|c|}
\hline & & \multicolumn{3}{|c|}{ Women } & \multicolumn{5}{|c|}{ Men } & \multirow[b]{3}{*}{ TOTAL } \\
\hline & & \multicolumn{2}{|c|}{ Age Group } & \multirow[b]{2}{*}{ TOTAL } & \multicolumn{4}{|c|}{ Age Group } & \multirow[b]{2}{*}{ TOTAL } & \\
\hline & & $40-49$ & $50+$ & & $16-29$ & $30-39$ & $40-49$ & $50+$ & & \\
\hline Falls & E880-E888 & 0 & 2 & 2 & 0 & 0 & 0 & 3 & 3 & 5 \\
\hline Fire & E890-E899 & 1 & 0 & 1 & 0 & 0 & 0 & 0 & 0 & 1 \\
\hline $\begin{array}{l}\text { Submersion/Suffocation/Foreign } \\
\text { Bodies }\end{array}$ & E910-E915 & 0 & 0 & 0 & 0 & 0 & 1 & 0 & 1 & 1 \\
\hline Other Accidents & E916-E928 & 1 & 4 & 5 & 3 & 1 & 4 & 10 & 18 & 23 \\
\hline
\end{tabular}

*Only those accident types and gender/age combinations with at least one occurrence appear in this table. 
OSHA Data

Appendix R. Number of Workdays Lost or with Restricted Activity in Each Accident Category by Gender and Age*

\begin{tabular}{|c|c|c|c|c|c|}
\hline & & \multicolumn{4}{|c|}{ Women } \\
\hline & & \multicolumn{4}{|c|}{ Age Group } \\
\hline & & \multicolumn{2}{|c|}{$40-49$} & \multicolumn{2}{|c|}{$50+$} \\
\hline & & $\begin{array}{c}\text { Days } \\
\text { Restricted }\end{array}$ & Days Lost & $\begin{array}{c}\text { Days } \\
\text { Restricted }\end{array}$ & Days Lost \\
\hline Type of Accident & E Codes & \multirow[b]{2}{*}{0} & \multirow[b]{2}{*}{0} & \multirow[b]{2}{*}{8} & \multirow[b]{2}{*}{110} \\
\hline Falls & \begin{tabular}{|l|} 
E880-E888 \\
\end{tabular} & & & & \\
\hline Fire & E890-E899 & 0 & 0 & 0 & 0 \\
\hline Natural/Environmental Factors & E900-E909 & 0 & 0 & 0 & 0 \\
\hline Other Accidents & E916-E928 & 0 & 0 & 6 & 183 \\
\hline
\end{tabular}

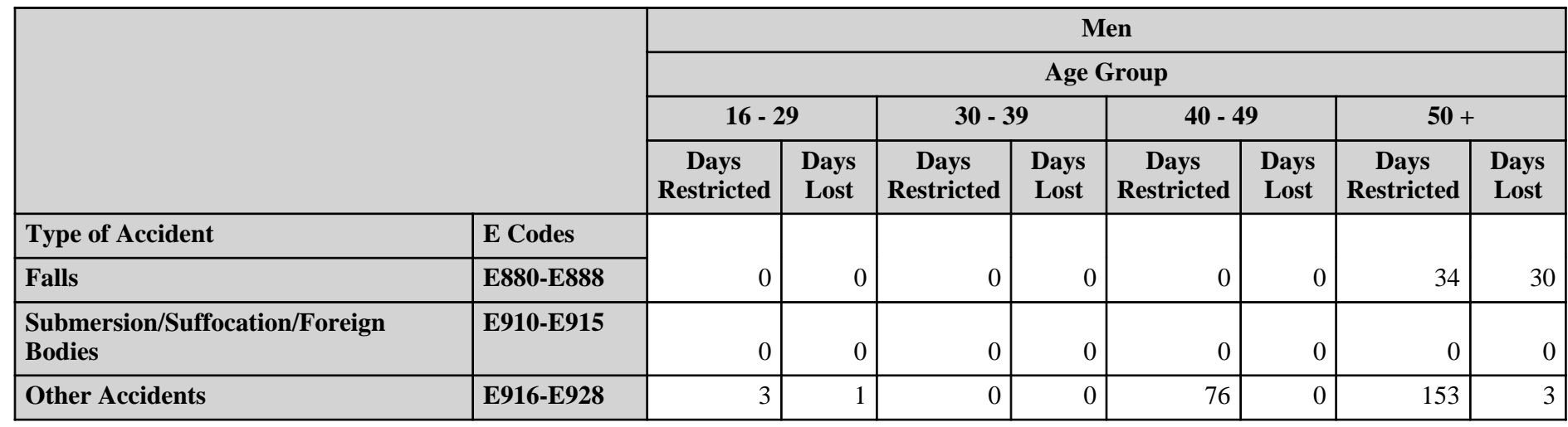

*OSHA events with $>1$ E code in the same accident type were counted only once. Only those accident types and gender/age combinations with at least one occurrence appear in this table. 


\section{Argonne National Laboratory 2009}

OSHA Data

Appendix S. Number of Diagnoses in Each Diagnostic Category by Gender and Job Category*

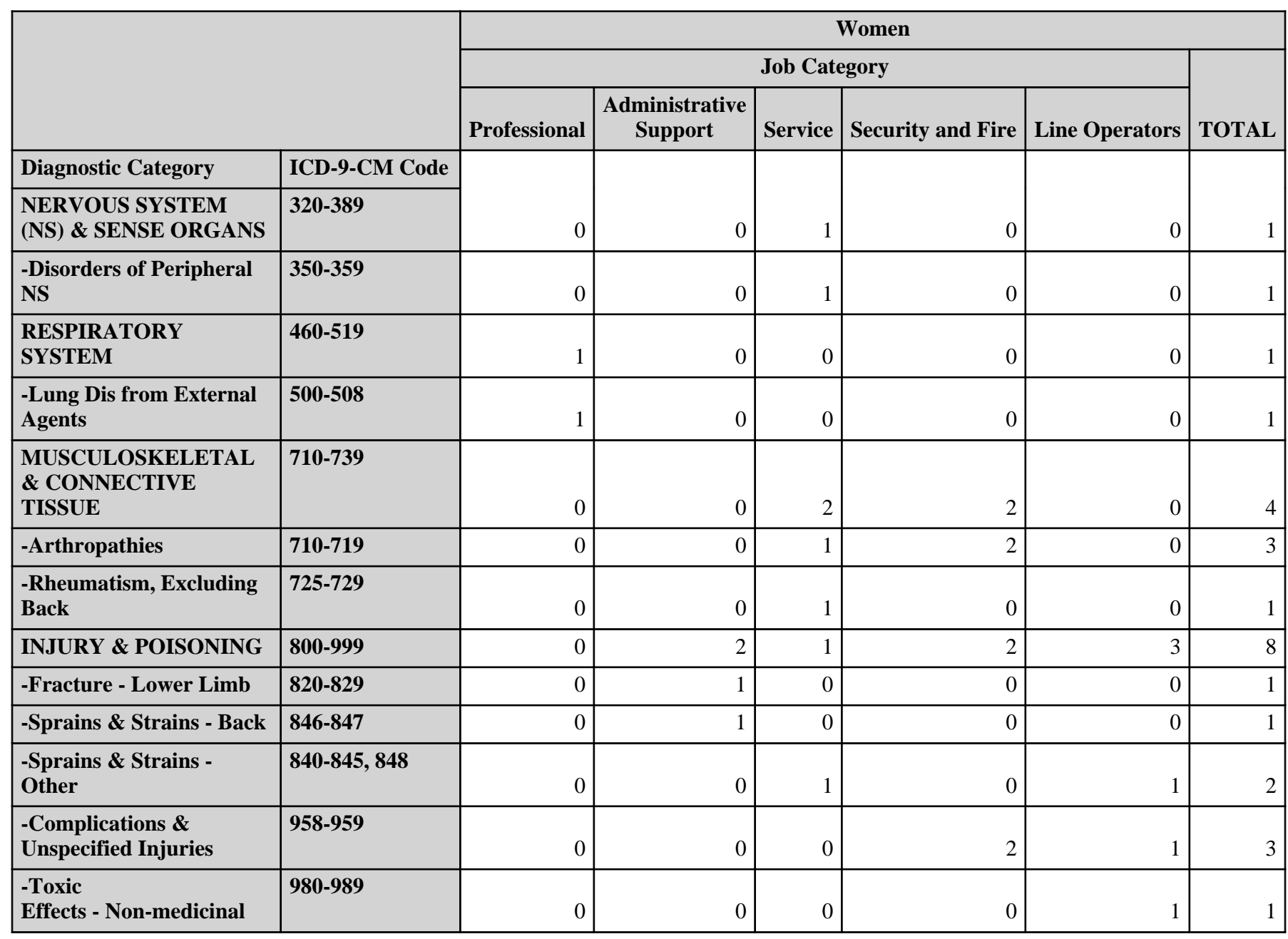

\begin{tabular}{|c|c|c|c|c|c|c|}
\hline & \multicolumn{6}{|c|}{ Women } \\
\hline & \multicolumn{5}{|c|}{ Job Category } & \multirow[b]{2}{*}{ TOTAL } \\
\hline & Professional & $\begin{array}{c}\text { Administrative } \\
\text { Support }\end{array}$ & Service & Security and Fire & Line Operators & \\
\hline Diagnostic Category & \multirow[b]{2}{*}{1} & \multirow[b]{2}{*}{2} & \multirow[b]{2}{*}{4} & \multirow[b]{2}{*}{4} & \multirow[b]{2}{*}{3} & \multirow[b]{2}{*}{14} \\
\hline Total & & & & & & \\
\hline
\end{tabular}

*Only those diagnostic categories and gender/job category combinations with at least one occurrence appear in this table. 


\section{Argonne National Laboratory 2009}

OSHA Data

Appendix S. Number of Diagnoses in Each Diagnostic Category by Gender and Job Category*

\begin{tabular}{|c|c|c|c|c|c|c|c|c|}
\hline & & \multicolumn{7}{|c|}{ Men } \\
\hline & & \multicolumn{6}{|c|}{ Job Category } & \multirow[b]{2}{*}{ TOTAL } \\
\hline & & Professional & $\begin{array}{c}\text { Administrative } \\
\text { Support }\end{array}$ & Service & $\begin{array}{l}\text { Security and } \\
\text { Fire }\end{array}$ & Crafts & $\begin{array}{c}\text { Line } \\
\text { Operators }\end{array}$ & \\
\hline Diagnostic Category & ICD-9-CM Code & & & & & & & \\
\hline $\begin{array}{l}\text { MUSCULOSKELETAL } \\
\text { \& CONNECTIVE } \\
\text { TISSUE }\end{array}$ & $710-739$ & 1 & 0 & 1 & 0 & 4 & 0 & 6 \\
\hline -Arthropathies & $710-719$ & 1 & 0 & 1 & 0 & 2 & 0 & 4 \\
\hline -Dorsopathies & $720-724$ & 0 & 0 & 0 & 0 & 1 & 0 & 1 \\
\hline $\begin{array}{l}\text {-Rheumatism, Excluding } \\
\text { Back }\end{array}$ & 725-729 & 0 & 0 & 0 & 0 & 1 & 0 & 1 \\
\hline $\begin{array}{l}\text { SYMPTOMS, } \\
\text { SIGNS, \& ILL-DEFINED } \\
\text { CONDITIONS }\end{array}$ & 780-799 & 0 & 0 & 1 & 0 & 0 & 0 & 1 \\
\hline -Symptoms & 780-789 & 0 & 0 & 1 & 0 & 0 & 0 & 1 \\
\hline INJURY \& POISONING & 800-999 & 4 & 2 & 6 & 2 & 7 & 1 & 22 \\
\hline -Fracture - Upper Limb & 810-819 & 1 & 0 & 0 & 1 & 0 & 0 & 2 \\
\hline -Fracture - Lower Limb & $820-829$ & 0 & 0 & 0 & 1 & 0 & 0 & 1 \\
\hline $\begin{array}{l}\text {-Sprains \& Strains - } \\
\text { Other }\end{array}$ & $840-845,848$ & 2 & 0 & 2 & 0 & 4 & 0 & 8 \\
\hline -Intracranial Injury & $850-854$ & 1 & 0 & 0 & 0 & 0 & 0 & 1 \\
\hline $\begin{array}{l}\text {-Open Wound - Upper } \\
\text { Limb }\end{array}$ & $880-887$ & 0 & 1 & 3 & 0 & 0 & 1 & 5 \\
\hline -Contusion & 920-924 & 0 & 0 & 1 & 0 & 0 & 0 & 1 \\
\hline $\begin{array}{l}\text {-Foreign Body Entering } \\
\text { Orifice }\end{array}$ & 930-939 & 0 & 0 & 0 & 0 & 1 & 0 & 1 \\
\hline $\begin{array}{l}\text {-Complications \& } \\
\text { Unspecified Injuries }\end{array}$ & 958-959 & 0 & 1 & 0 & 0 & 2 & 0 & 3 \\
\hline
\end{tabular}

\begin{tabular}{|c|c|c|c|c|c|c|c|}
\hline & \multicolumn{7}{|c|}{ Men } \\
\hline & \multicolumn{6}{|c|}{ Job Category } & \multirow[b]{2}{*}{ TOTAL } \\
\hline & Professional & $\begin{array}{l}\text { Administrative } \\
\text { Support }\end{array}$ & Service & Security and Fire & Crafts & Line Operators & \\
\hline Diagnostic Category & \multirow[b]{2}{*}{5} & \multirow[b]{2}{*}{2} & \multirow[b]{2}{*}{8} & \multirow[b]{2}{*}{2} & \multirow[b]{2}{*}{11} & \multirow[b]{2}{*}{1} & \multirow[b]{2}{*}{29} \\
\hline Total & & & & & & & \\
\hline
\end{tabular}

*Only those diagnostic categories and gender/job category combinations with at least one occurrence appear in this table. 
Appendix T. Number of Workdays Lost or with Restricted Activity in Each Diagnostic Category by Gender and Job Category*

\begin{tabular}{|c|c|c|c|c|c|}
\hline & & \multicolumn{4}{|c|}{ Women } \\
\hline & & \multicolumn{4}{|c|}{ Job Category } \\
\hline & & \multicolumn{2}{|c|}{ Professional } & \multicolumn{2}{|c|}{$\begin{array}{l}\text { Administrative } \\
\text { Support }\end{array}$} \\
\hline & & $\begin{array}{c}\text { Days } \\
\text { Restricted }\end{array}$ & Days Lost & $\begin{array}{c}\text { Days } \\
\text { Restricted }\end{array}$ & Days Lost \\
\hline Diagnostic Category & ICD-9-CM Codes & \multirow[b]{2}{*}{0} & \multirow[b]{2}{*}{0} & \multirow[b]{2}{*}{0} & \multirow[b]{2}{*}{0} \\
\hline -Disorders of Peripheral NS & $350-359$ & & & & \\
\hline -Lung Dis from External Agents & 500-508 & 0 & 0 & 0 & 0 \\
\hline -Arthropathies & $710-719$ & 0 & 0 & 0 & 0 \\
\hline -Rheumatism, Excluding Back & 725-729 & 0 & 0 & 0 & 0 \\
\hline -Fracture - Lower Limb & $820-829$ & 0 & 0 & 0 & 0 \\
\hline -Sprains \& Strains - Back & 846-847 & 0 & 0 & 0 & 3 \\
\hline -Sprains \& Strains - Other & $840-845,848$ & 0 & 0 & 0 & 0 \\
\hline $\begin{array}{l}\text {-Complications \& Unspecified } \\
\text { Injuries }\end{array}$ & 958-959 & 0 & 0 & 0 & 0 \\
\hline -Toxic Effects - Non-medicinal & 980-989 & 0 & 0 & 0 & 0 \\
\hline
\end{tabular}

\begin{tabular}{|c|c|c|c|c|c|c|c|}
\hline & & \multicolumn{6}{|c|}{ Women } \\
\hline & & \multicolumn{6}{|c|}{ Job Category } \\
\hline & & \multicolumn{2}{|c|}{ Service } & \multicolumn{2}{|c|}{ Security and Fire } & \multicolumn{2}{|c|}{ Line Operators } \\
\hline & & $\begin{array}{c}\text { Days } \\
\text { Restricted }\end{array}$ & Days Lost & $\begin{array}{c}\text { Days } \\
\text { Restricted }\end{array}$ & Days Lost & $\begin{array}{c}\text { Days } \\
\text { Restricted }\end{array}$ & Days Lost \\
\hline Diagnostic Category & ICD-9-CM Codes & \multirow[b]{2}{*}{2} & \multirow[b]{2}{*}{180} & \multirow[b]{2}{*}{0} & \multirow[b]{2}{*}{0} & \multirow[b]{2}{*}{0} & \multirow[b]{2}{*}{0} \\
\hline -Disorders of Peripheral NS & $350-359$ & & & & & & \\
\hline -Lung Dis from External Agents & $500-508$ & 0 & 0 & 0 & 0 & 0 & $\overline{0}$ \\
\hline -Arthropathies & 710-719 & 2 & 180 & 0 & 0 & 0 & $\overline{0}$ \\
\hline -Rheumatism, Excluding Back & 725-729 & 2 & 180 & 0 & 0 & 0 & 0 \\
\hline -Fracture - Lower Limb & $820-829$ & 0 & 0 & 0 & 0 & 0 & 0 \\
\hline -Sprains \& Strains - Back & 846-847 & 0 & 0 & 0 & 0 & 0 & 0 \\
\hline -Sprains \& Strains - Other & $840-845,848$ & 8 & 110 & 0 & 0 & 4 & 0 \\
\hline $\begin{array}{l}\text {-Complications \& Unspecified } \\
\text { Injuries }\end{array}$ & 958-959 & 0 & 0 & 0 & 0 & 0 & 0 \\
\hline -Toxic Effects - Non-medicinal & 980-989 & 0 & 0 & 0 & 0 & 0 & 0 \\
\hline
\end{tabular}

*OSHA events with >1 ICD-9-CM code in the same diagnostic category were counted only once. Only those diagnostic categories and gender/job category combinations with at least one occurrence appear in this table. 


\section{Argonne National Laboratory 2009}

\section{OSHA Data}

Appendix T. Number of Workdays Lost or with Restricted Activity in Each Diagnostic Category by Gender and Job Category*

\begin{tabular}{|c|c|c|c|c|c|c|c|}
\hline & & \multicolumn{6}{|c|}{ Men } \\
\hline & & \multicolumn{6}{|c|}{ Job Category } \\
\hline & & \multicolumn{2}{|c|}{ Professional } & \multicolumn{2}{|c|}{$\begin{array}{l}\text { Administrative } \\
\text { Support }\end{array}$} & \multicolumn{2}{|c|}{ Service } \\
\hline & & $\begin{array}{c}\text { Days } \\
\text { Restricted }\end{array}$ & Days Lost & \begin{tabular}{|c|} 
Days \\
Restricted
\end{tabular} & Days Lost & $\begin{array}{c}\text { Days } \\
\text { Restricted }\end{array}$ & Days Lost \\
\hline Diagnostic Category & ICD-9-CM Codes & & & & & & \\
\hline -Arthropathies & 710-719 & 10 & 0 & 0 & 0 & 6 & 0 \\
\hline -Dorsopathies & $720-724$ & 0 & 0 & 0 & 0 & 0 & 0 \\
\hline -Rheumatism, Excluding Back & 725-729 & 0 & 0 & 0 & 0 & 0 & 0 \\
\hline -Symptoms & 780-789 & 0 & 0 & 0 & 0 & 0 & 1 \\
\hline -Fracture - Upper Limb & $810-819$ & 25 & 0 & 0 & 0 & 0 & 0 \\
\hline -Fracture - Lower Limb & $820-829$ & 0 & 0 & 0 & 0 & 0 & 0 \\
\hline -Sprains \& Strains - Other & $840-845,848$ & 10 & 0 & 0 & 0 & 1 & 0 \\
\hline -Intracranial Injury & $850-854$ & 23 & 21 & 0 & 0 & 0 & 0 \\
\hline -Open Wound - Upper Limb & $880-887$ & 0 & 0 & 8 & 0 & 39 & 1 \\
\hline -Contusion & 920-924 & 0 & 0 & 0 & 0 & 0 & 0 \\
\hline -Foreign Body Entering Orifice & 930-939 & 0 & 0 & 0 & 0 & 0 & 0 \\
\hline $\begin{array}{l}\text {-Complications \& Unspecified } \\
\text { Injuries }\end{array}$ & 958-959 & 0 & 0 & 18 & 0 & 0 & 0 \\
\hline
\end{tabular}

\begin{tabular}{|c|c|c|c|c|c|c|c|}
\hline & & \multicolumn{6}{|c|}{ Men } \\
\hline & & \multicolumn{6}{|c|}{ Job Category } \\
\hline & & \multicolumn{2}{|c|}{ Security and Fire } & \multicolumn{2}{|c|}{ Crafts } & \multicolumn{2}{|c|}{ Line Operators } \\
\hline & & \begin{tabular}{|c|} 
Days \\
Restricted
\end{tabular} & Days Lost & \begin{tabular}{c|} 
Days \\
Restricted
\end{tabular} & Days Lost & \begin{tabular}{c|c|} 
Days \\
Restricted
\end{tabular} & Days Lost \\
\hline Diagnostic Category & ICD-9-CM Codes & & & & & & \\
\hline -Arthropathies & $710-719$ & 0 & 0 & 28 & 3 & 0 & 0 \\
\hline -Dorsopathies & $720-724$ & 0 & 0 & 2 & 0 & 0 & $\overline{0}$ \\
\hline -Rheumatism, Excluding Back & $725-729$ & 0 & 0 & 28 & 3 & 0 & 0 \\
\hline -Symptoms & $780-789$ & 0 & 0 & 0 & 0 & 0 & 0 \\
\hline -Fracture - Upper Limb & $810-819$ & 56 & 0 & 0 & 0 & 0 & 0 \\
\hline -Fracture - Lower Limb & $820-829$ & 39 & 0 & 0 & 0 & 0 & 0 \\
\hline -Sprains \& Strains - Other & $840-845,848$ & 0 & 0 & 28 & 3 & 0 & 0 \\
\hline -Intracranial Injury & $850-854$ & 0 & 0 & 0 & 0 & 0 & 0 \\
\hline -Open Wound - Upper Limb & $880-887$ & 0 & 0 & 0 & 0 & 0 & 0 \\
\hline -Contusion & $920-924$ & 0 & 0 & 0 & 0 & 0 & 0 \\
\hline -Foreign Body Entering Orifice & $930-939$ & 0 & 0 & 0 & 0 & 0 & 0 \\
\hline $\begin{array}{l}\text {-Complications \& Unspecified } \\
\text { Injuries }\end{array}$ & 958-959 & 0 & 0 & 13 & 9 & 0 & 0 \\
\hline
\end{tabular}

*OSHA events with >1 ICD-9-CM code in the same diagnostic category were counted only once. Only those diagnostic categories and gender/job category combinations with at least one occurrence appear in this table. 
Argonne National Laboratory 2009

OSHA Data

Appendix U. Number of Occurrences in Each Accident Category by Gender and Job Category*

\begin{tabular}{|c|c|c|c|c|c|c|c|}
\hline & & \multicolumn{6}{|c|}{ Women } \\
\hline & & \multicolumn{5}{|c|}{ Job Category } & \multirow[b]{2}{*}{ TOTAL } \\
\hline & & Professional & $\begin{array}{c}\text { Administrative } \\
\text { Support }\end{array}$ & Service & \begin{tabular}{|} 
Security and \\
Fire
\end{tabular} & \begin{tabular}{|c|} 
Line \\
Operators
\end{tabular} & \\
\hline Type of Accident & E CODES & \multirow[b]{2}{*}{0} & \multirow[b]{2}{*}{1} & \multirow[b]{2}{*}{1} & \multirow[b]{2}{*}{0} & \multirow[b]{2}{*}{0} & \multirow[b]{2}{*}{2} \\
\hline Falls & E880-E888 & & & & & & \\
\hline Natural/Environmental Factors & E900-E909 & 0 & 0 & 0 & 0 & 1 & 1 \\
\hline Other Accidents & E916-E928 & 0 & 1 & 1 & 1 & 2 & 5 \\
\hline
\end{tabular}

\begin{tabular}{|c|c|c|c|c|c|c|c|c|}
\hline & & \multicolumn{7}{|c|}{ Men } \\
\hline & & \multicolumn{6}{|c|}{ Job Category } & \multirow[b]{2}{*}{ TOTAL } \\
\hline & & Professional & $\begin{array}{c}\text { Administrative } \\
\text { Support }\end{array}$ & Service & $\begin{array}{l}\text { Security } \\
\text { and Fire }\end{array}$ & Crafts & $\begin{array}{c}\text { Line } \\
\text { Operators }\end{array}$ & \\
\hline Type of Accident & E CODES & \multirow[b]{2}{*}{1} & \multirow[b]{2}{*}{0} & \multirow[b]{2}{*}{0} & \multirow[b]{2}{*}{0} & \multirow[b]{2}{*}{2} & \multirow[b]{2}{*}{0} & \multirow[b]{2}{*}{3} \\
\hline Falls & E880-E888 & & & & & & & \\
\hline $\begin{array}{l}\text { Submersion/Suffocation/Foreign } \\
\text { Bodies }\end{array}$ & E910-E915 & 0 & 0 & 0 & 0 & 1 & 0 & 1 \\
\hline Other Accidents & E916-E928 & 3 & 2 & 7 & 2 & 3 & 1 & 18 \\
\hline
\end{tabular}

*Only those accident types and gender/job category combinations with at least one occurrence appear in this table. 
OSHA Data

Appendix V. Number of Workdays Lost or with Restricted Activity in Each Accident Category by Gender and Job Category*

\begin{tabular}{|c|c|c|c|c|c|}
\hline & & \multicolumn{4}{|c|}{ Women } \\
\hline & & \multicolumn{4}{|c|}{ Job Category } \\
\hline & & \multicolumn{2}{|c|}{ Professional } & \multicolumn{2}{|c|}{$\begin{array}{c}\text { Administrative } \\
\text { Support }\end{array}$} \\
\hline & & $\begin{array}{c}\text { Days } \\
\text { Restricted }\end{array}$ & Days Lost & $\begin{array}{c}\text { Days } \\
\text { Restricted }\end{array}$ & Days Lost \\
\hline Type of Accident & E Codes & \multirow[b]{2}{*}{0} & \multirow[b]{2}{*}{0} & \multirow[b]{2}{*}{0} & \multirow[b]{2}{*}{0} \\
\hline Falls & E880-E888 & & & & \\
\hline Fire & E890-E899 & 0 & 0 & 0 & 0 \\
\hline Natural/Environmental Factors & E900-E909 & 0 & 0 & 0 & 0 \\
\hline Other Accidents & E916-E928 & 0 & 0 & 0 & 3 \\
\hline
\end{tabular}

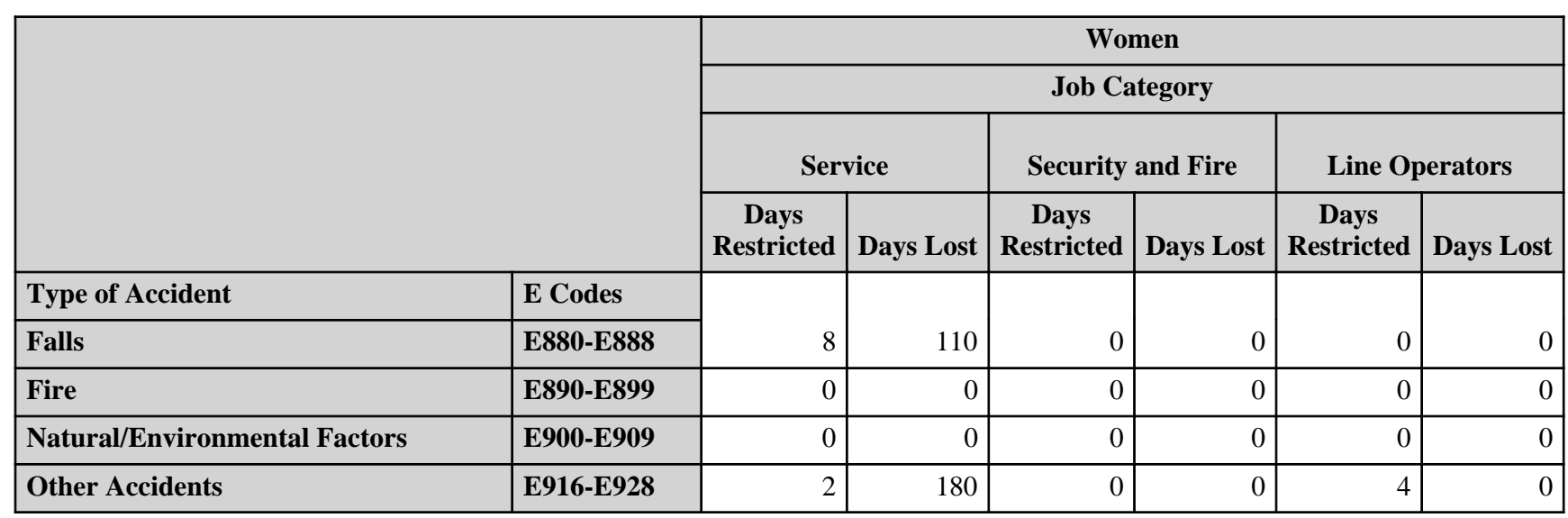

*OSHA events with >1 E code in the same accident type were counted only once. Only those accident types and gender/job category combinations with at least one occurrence appear in this table. 
OSHA Data

Appendix V. Number of Workdays Lost or with Restricted Activity in Each Accident Category by Gender and Job Category*

\begin{tabular}{|c|c|c|c|c|c|c|c|}
\hline & & \multicolumn{6}{|c|}{ Men } \\
\hline & & \multicolumn{6}{|c|}{ Job Category } \\
\hline & & \multicolumn{2}{|c|}{ Professional } & \multicolumn{2}{|c|}{$\begin{array}{l}\text { Administrative } \\
\text { Support }\end{array}$} & \multicolumn{2}{|c|}{ Service } \\
\hline & & $\begin{array}{c}\text { Days } \\
\text { Restricted }\end{array}$ & Days Lost & \begin{tabular}{c|} 
Days \\
Restricted
\end{tabular} & Days Lost & \begin{tabular}{c|} 
Days \\
Restricted
\end{tabular} & Days Lost \\
\hline Type of Accident & E Codes & \multirow[b]{2}{*}{23} & \multirow[b]{2}{*}{21} & \multirow[b]{2}{*}{0} & \multirow[b]{2}{*}{0} & \multirow[b]{2}{*}{0} & \multirow[b]{2}{*}{0} \\
\hline Falls & \begin{tabular}{|l|} 
E880-E888 \\
\end{tabular} & & & & & & \\
\hline $\begin{array}{l}\text { Submersion/Suffocation/Foreign } \\
\text { Bodies }\end{array}$ & \begin{tabular}{|l|} 
E910-E915 \\
\end{tabular} & 0 & 0 & 0 & 0 & 0 & 0 \\
\hline Other Accidents & E916-E928 & 35 & 0 & 26 & 0 & 46 & 1 \\
\hline
\end{tabular}

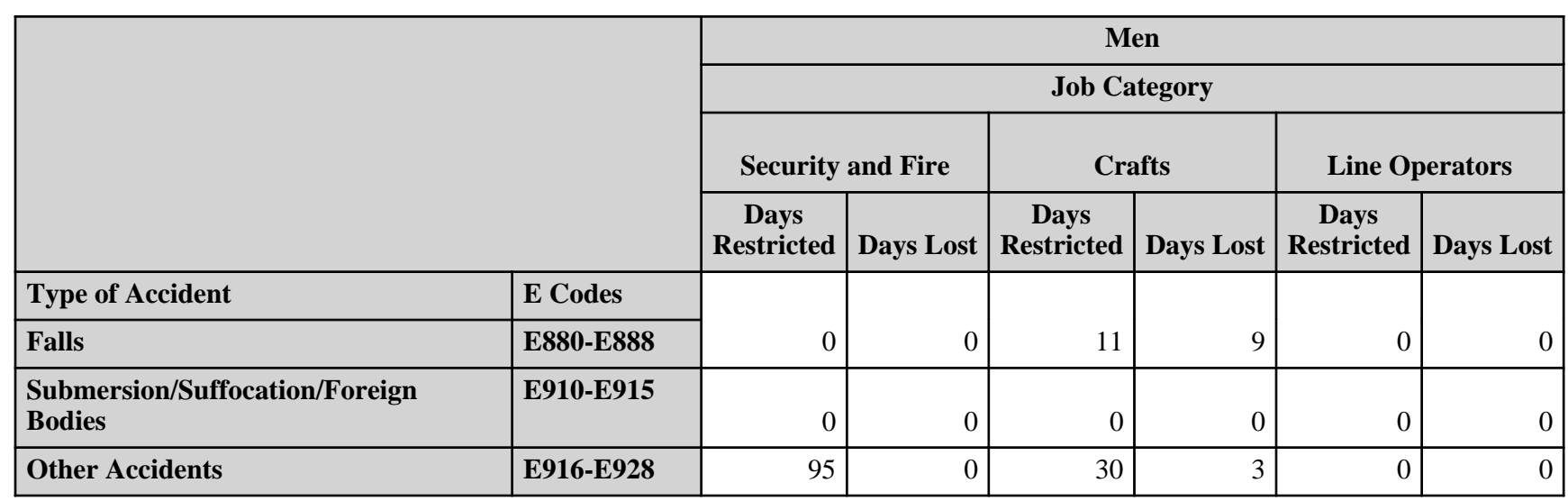

*OSHA events with >1 E code in the same accident type were counted only once. Only those accident types and gender/job category combinations with at least one occurrence appear in this table. 
Argonne National Laboratory 2009

OSHA Data

Appendix W. Age-Adjusted OSHA Illness and Injury Rates by Diagnostic Category*

Part 1. Men

\begin{tabular}{|c|c|c|c|c|c|}
\hline & & $\begin{array}{l}\text { Number of } \\
\text { Diagnoses }\end{array}$ & $\begin{array}{l}\text { Age-Adjusted } \\
\text { Rate per } 1,000 * *\end{array}$ & \begin{tabular}{|c|} 
Lower $95 \%$ \\
Confidence \\
Limit per 1,000
\end{tabular} & $\begin{array}{c}\text { Upper } 95 \% \\
\text { Confidence } \\
\text { Limit per } 1,000\end{array}$ \\
\hline Diagnostic Category & ICD-9-CM Code & & & & \\
\hline $\begin{array}{l}\text { MUSCULOSKELETAL \& CONNECTIVE } \\
\text { TISSUE }\end{array}$ & 710-739 & 6 & 3.9 & 1.1 & 14.1 \\
\hline -Arthropathies & $710-719$ & 4 & 1.1 & 0.4 & 3.0 \\
\hline -Dorsopathies & $720-724$ & 1 & 2.5 & 0.3 & 17.6 \\
\hline -Rheumatism, Excluding Back & 725-729 & 1 & 0.3 & 0.0 & 2.0 \\
\hline $\begin{array}{l}\text { SYMPTOMS, SIGNS, \& ILL-DEFINED } \\
\text { CONDITIONS }\end{array}$ & 780-799 & 1 & 2.5 & 0.3 & 17.6 \\
\hline -Symptoms & 780-789 & 1 & 2.5 & 0.3 & 17.6 \\
\hline INJURY \& POISONING & $800-999$ & 22 & 13.9 & 7.3 & 26.5 \\
\hline -Fracture - Upper Limb & 810-819 & 2 & 0.7 & 0.2 & 2.9 \\
\hline -Fracture - Lower Limb & $820-829$ & 1 & 0.3 & 0.0 & 2.0 \\
\hline -Sprains \& Strains - Other & $840-845,848$ & 8 & 4.6 & 1.5 & 14.0 \\
\hline -Open Wound - Upper Limb & $880-887$ & 5 & 4.4 & 1.3 & 14.5 \\
\hline -Contusion & $920-924$ & 1 & 0.3 & 0.0 & 2.0 \\
\hline -Foreign Body Entering Orifice & $930-939$ & 1 & 0.4 & 0.1 & 3.0 \\
\hline -Complications of Surgical/Medical Care & 996-999 & 4 & 3.3 & 0.7 & 14.8 \\
\hline Total & & 29 & 20.2 & 11.6 & 35.5 \\
\hline
\end{tabular}

* Only those diagnostic categories with at least one occurrence appear in this table.

**Standardized to age distribution of 2000 U.S. population. 
Argonne National Laboratory 2009

OSHA Data

Appendix W. Age-Adjusted OSHA Illness and Injury Rates by Diagnostic Category*

Part 2. Women

\begin{tabular}{|c|c|c|c|c|c|}
\hline & & $\begin{array}{l}\text { Number of } \\
\text { Diagnoses }\end{array}$ & $\begin{array}{c}\text { Age-Adjusted } \\
\text { Rate per } 1,000 * *\end{array}$ & $\begin{array}{c}\text { Lower } 95 \% \\
\text { Confidence } \\
\text { Limit per 1,000 }\end{array}$ & $\begin{array}{c}\text { Upper } 95 \% \\
\text { Confidence } \\
\text { Limit per } 1,000\end{array}$ \\
\hline Diagnostic Category & ICD-9-CM Code & \multirow[b]{2}{*}{1} & \multirow[b]{2}{*}{0.6} & \multirow[b]{2}{*}{0.1} & \multirow[b]{2}{*}{4.1} \\
\hline NERVOUS SYSTEM (NS) \& SENSE ORGANS & 320-389 & & & & \\
\hline -Disorders of Peripheral NS & $350-359$ & 1 & 0.6 & 0.1 & 4.1 \\
\hline RESPIRATORY SYSTEM & $460-519$ & 1 & 0.9 & 0.1 & 6.6 \\
\hline -Lung Dis from External Agents & $500-508$ & 1 & 0.9 & 0.1 & 6.6 \\
\hline $\begin{array}{l}\text { MUSCULOSKELETAL \& CONNECTIVE } \\
\text { TISSUE }\end{array}$ & $710-739$ & 4 & 3.0 & 1.1 & 8.2 \\
\hline -Arthropathies & 710-719 & 3 & 2.4 & 0.8 & 7.7 \\
\hline -Rheumatism, Excluding Back & $725-729$ & 1 & 0.6 & 0.1 & 4.1 \\
\hline INJURY \& POISONING & $800-999$ & 8 & 7.0 & 3.4 & 14.1 \\
\hline -Fracture - Lower Limb & $820-829$ & 1 & 1.0 & 0.1 & 7.2 \\
\hline -Sprains \& Strains - Back & 846-847 & 1 & 1.0 & 0.1 & 7.2 \\
\hline -Sprains \& Strains - Other & $840-845,848$ & 2 & 1.6 & 0.4 & 6.7 \\
\hline -Toxic Effects - Non-medicinal & $980-989$ & 1 & 0.9 & 0.1 & 6.6 \\
\hline -Complications of Surgical/Medical Care & 996-999 & 3 & 2.4 & 0.8 & 7.7 \\
\hline Total & & 14 & 11.5 & 6.7 & 19.7 \\
\hline
\end{tabular}

* Only those diagnostic categories with at least one occurrence appear in this table.

**Standardized to age distribution of 2000 U.S. population. 
Argonne National Laboratory 2009

OSHA Data

Appendix W. Age-Adjusted OSHA Illness and Injury Rates by Diagnostic Category*

Part 3. Men and Women

\begin{tabular}{|c|c|c|c|c|c|}
\hline & & $\begin{array}{l}\text { Number of } \\
\text { Diagnoses }\end{array}$ & $\begin{array}{c}\text { Age-Adjusted } \\
\text { Rate per } 1,000 * *\end{array}$ & \begin{tabular}{|c|} 
Lower $95 \%$ \\
Confidence \\
Limit per 1,000
\end{tabular} & $\begin{array}{c}\text { Upper } 95 \% \\
\text { Confidence } \\
\text { Limit per 1,000 }\end{array}$ \\
\hline Diagnostic Category & ICD-9-CM Code & & & & \\
\hline NERVOUS SYSTEM (NS) \& SENSE ORGANS & 320-389 & 1 & 0.2 & 0.0 & 1.3 \\
\hline -Disorders of Peripheral NS & 350-359 & 1 & 0.2 & 0.0 & 1.3 \\
\hline RESPIRATORY SYSTEM & $460-519$ & 1 & 0.3 & 0.0 & 2.0 \\
\hline -Lung Dis from External Agents & $500-508$ & 1 & 0.3 & 0.0 & 2.0 \\
\hline $\begin{array}{l}\text { MUSCULOSKELETAL \& CONNECTIVE } \\
\text { TISSUE }\end{array}$ & 710-739 & 10 & 3.4 & 1.3 & 8.7 \\
\hline -Arthropathies & 710-719 & 7 & 1.5 & 0.7 & 3.2 \\
\hline -Dorsopathies & $720-724$ & 1 & 1.5 & 0.2 & 10.6 \\
\hline -Rheumatism, Excluding Back & 725-729 & 2 & 0.4 & 0.1 & 1.5 \\
\hline $\begin{array}{l}\text { SYMPTOMS, SIGNS, \& ILL-DEFINED } \\
\text { CONDITIONS }\end{array}$ & 780-799 & 1 & 1.5 & 0.2 & 10.6 \\
\hline -Symptoms & 780-789 & 1 & 1.5 & 0.2 & 10.6 \\
\hline INJURY \& POISONING & $800-999$ & 30 & 11.0 & 6.5 & 18.4 \\
\hline -Fracture - Upper Limb & 810-819 & 2 & 0.5 & 0.1 & 2.0 \\
\hline -Fracture - Lower Limb & $820-829$ & 2 & 0.5 & 0.1 & 1.8 \\
\hline -Sprains \& Strains - Back & 846-847 & 1 & 0.2 & 0.0 & 1.6 \\
\hline -Sprains \& Strains - Other & $840-845,848$ & 10 & 3.3 & 1.3 & 8.7 \\
\hline -Open Wound - Upper Limb & 880-887 & 5 & 2.8 & 0.9 & 8.9 \\
\hline -Contusion & $920-924$ & 1 & 0.2 & 0.0 & 1.3 \\
\hline -Foreign Body Entering Orifice & 930-939 & 1 & 0.3 & 0.0 & 2.0 \\
\hline -Toxic Effects - Non-medicinal & 980-989 & 1 & 0.3 & 0.0 & 2.0 \\
\hline -Complications of Surgical/Medical Care & 996-999 & 7 & 2.9 & 1.0 & 8.6 \\
\hline Total & & 43 & 16.3 & 10.5 & 25.4 \\
\hline
\end{tabular}

* Only those diagnostic categories with at least one occurrence appear in this table.

**Standardized to age distribution of 2000 U.S. population. 